\title{
Kinesin-3 mediated axonal delivery of presynaptic neurexin stabilizes dendritic spines and postsynaptic components
}

Devyn Oliver ${ }^{1}$, Shankar Ramachandran ${ }^{1}$, Alison Philbrook ${ }^{1}$, Christopher M. Lambert ${ }^{1}$, Ken C. Q. Nguyen $^{2}$, David H. Hall ${ }^{2},{ }^{*}$ Michael M. Francis ${ }^{1}$

${ }^{1}$ Department of Neurobiology, University of Massachusetts Medical School, Worcester, MA 01605

${ }^{2}$ Department of Neuroscience, Albert Einstein College of Medicine, Bronx, NY 10461

*Corresponding author: michael.francis@umassmed.edu

Key words:

dendritic spines, neurexin, kinesin, C. elegans, synapse development, synaptic maintenance, active zone, protein trafficking 


\section{Contributions Summary}

D.O. generated strains, transgenic lines, molecular constructs, confocal and electron microscopy images, and data analysis. S.R. performed all calcium imaging experiments and analysis. C.M.L. generated destination vectors and $n r x-1$ constructs. D.H.H. and K.C.Q.N. aided in electron microscopy experiments and analysis. M.M.F and D.O designed and interpreted results of all experiments and wrote the manuscript.

\section{Acknowledgements}

Nematode strains were provided by the Caenorhabditis Genetics Center which is funded by the NIH National Center for Research Resources. Thank you to Dori Schafer for access to 3D rendering Imaris software. Thank you to Dirk Albrecht's laboratory for advice with hydrogel immobilization. Thank you to the laboratories of: Dong Yan, Kang Shen, Andres Maricq, Michael Nonet, Peri Kurshan, and Gert Jansen for providing reagents. Thank you to Peri Kurshan for sharing unpublished results. Thank you to William Joyce and Michael Gorczyca for technical assistance. Thank you to Steve Cook for help with analysis of electron micrographs and John White for use of N2U electron micrographs. Lastly, we thank members of the Francis laboratory for manuscript comments.

\section{Funding}

This research was supported by NIH NINDS RO1NS064263 (MMF), R21NS101649 (MMF), NIH OD $01943(\mathrm{DHH})$, and F31NS103365 (DO). The Philips CM10 TEM electron microscope used in this study was acquired through a NIH Shared Instrumentation Grant (1S100D016214-01A1) at Albert Einstein College of Medicine. 


\begin{abstract}
The functional properties of neural circuits are defined by the patterns of synaptic connections between their partnering neurons, but the mechanisms that stabilize circuit connectivity are poorly understood. We systemically examined this question at synapses onto newly characterized dendritic spines of $C$. elegans GABAergic motor neurons. We show that the presynaptic adhesion protein, neurexin/NRX-1, is required for stabilization of postsynaptic structure. We find that early postsynaptic developmental events proceed without a strict requirement for synaptic activity and are not disrupted by deletion of neurexin/nrx-1. However, in the absence of presynaptic NRX-1, dendritic spines and receptor clusters become destabilized and collapse prior to adulthood. We demonstrate that kinesin-3/UNC-104 delivers NRX-1 to presynaptic terminals and show that ongoing delivery is required for postsynaptic maintenance in mature animals. By defining the dynamics and temporal order of synapse formation events in vivo, we describe a mechanism for stabilizing mature circuit connectivity through neurexinbased adhesion.
\end{abstract}




\section{Introduction}

The capabilities of neural circuits to perform specific functions arise from the patterns of synaptic connections between their partnering neurons. The organization of these connections is circuit-specific and established through a complex process that involves the coordinated assembly and maturation of specialized pre- and postsynaptic structures on appropriate partnering neurons, and their maintenance in the mature nervous system. We now have a generalized understanding of synapse structure. Active zone (AZ) proteins and neurotransmitter-filled synaptic vesicles position near the presynaptic membrane for rapid release while neurotransmitter receptors are clustered at high density in apposition to these sites in order to ensure the fidelity of synaptic communication. Genetic studies have identifed numerous mutations that alter circuit connectivity or affect the overall structural organization of synapses (Hendi et al., 2019; Richmond et al., 1999; Zhen \& Jin, 1999). However, for many of the synapse-associated proteins affected by these mutations, we do not yet have a mechanistic understanding of their roles in establishing synaptic connections, or how their disruption may lead to alterations in synapse stability. Gaining an enhanced understanding of the sequence of events involved in synapse assembly, maturation, and maintenance, and their relative timing in vivo is critical for addressing these questions.

Several prior studies have examined molecular events during synaptogenesis. In vivo studies in C. elegans and Drosophila have largely focused on the formation of the presynaptic active zone. Collectively, these studies provide compelling evidence for a model where active zone assembly occurs sequentially. While there is some variability across synapse type, the early stages of this process are generally organized by the highly conserved synaptic scaffolds SYD-2/Liprin- $\alpha$ and SYD-1/Rho GTPase, which then recruit additional key conserved AZ proteins such as ELKS-1/Bruchpilot, Piccolo family members, and UNC-10/RIM for subsequent stages of assembly, including clustering of $\mathrm{Ca}^{2+}$ channels, and the recruitment of synaptic 
vesicles (Bohme et al., 2016; Dai et al., 2006; Fouquet et al., 2009; Patel et al., 2006; Zhen \& Jin, 1999). Remarkably, nascent AZs can assemble quite rapidly (within minutes) but then often undergo a more extended period of maturation that can last for several hours (Fouquet et al., 2009; Lipton et al., 2018; McDonald et al., 2020; Nagerl et al., 2007). The relationship of postsynaptic development to presynaptic assembly remains less clear. Live imaging studies of cultured mouse hippocampal neurons have suggested that the assembly of presynaptic components precedes recruitment of postsynaptic receptors and scaffolds (Friedman et al., 2000). More recently, 2-photon imaging of organotypic slice cultures from rat hippocampus showed that new spines accumulate glutamate receptors concurrently with their growth and are competent to participate in transmission within a few hours after outgrowth (Zito et al., 2009). Comparatively few in vivo studies have investigated coordinated pre- and postsynaptic development, and significant questions remain about molecular mechanisms that direct linkages between pre- and postsynaptic specializations to promote their maturation and stabilization.

Evolutionarily conserved synaptic adhesion proteins, such as neurexins, are prime candidates for coordinating pre- and postsynaptic events during synaptogenesis. Their importance is underscored by the fact that neurexin alterations are associated with cognitive disease (Sudhof, 2008). For example, mutations in human Neurexin1 are associated with several neuropsychiatric disorders, including schizophrenia and autism spectrum disorders (Kasem et al., 2018). Neurexins are typically localized presynaptically and linked to postsynaptic binding partners such as dystroglycans, LRRTMs, neuroligins, and cerebellins, through extracellular laminin and EGF-like repeats to establish transsynaptic connections. Neurexin mediated transsynaptic signaling has been implicated in key aspects of synapse development and function. For example, loss of presynaptic neurexin at the fly neuromuscular junction increases the length of the presynaptic density, and also alters the size and molecular composition of apposed muscle glutamate receptor clusters (J. Li et al., 2007) (Owald et al., 
2012). The broad brain distribution of neurexins and the many neurexin isoform variants in the nervous system enable complex, cell-type specific functions for neurexins at synapses (Missler \& Sudhof, 1998) (Sudhof, 2017). For example, conditional deletion of mouse neurexins revealed strikingly divergent functions across synapses formed by cortical inhibitory interneurons and those formed by cerebellar climbing fibers onto Purkinje neurons (L. Y. Chen et al., 2017). Importantly, molecular mechanisms for their trafficking and delivery to synapses also remain incompletely defined. Genetic tools that enable cell-type specific analysis are therefore critical for uncovering precise functional roles of synaptic adhesion proteins and mechanisms for their regulation within the context of individual neural circuits.

We previously identified finger-like protrusions from the dendritic processes of $C$. elegans DD GABAergic motor neurons (Figure 1A) (Philbrook et al., 2018). Characterization of these structures by our laboratory and others pointed towards the idea that they receive synaptic input from presynaptic cholinergic motor neurons and serve analogous roles to dendritic spines in the mammalian brain (J. G. White et al., 1976) (Oliver et al., 2018) (CuentasCondori et al., 2019). Deletion of $n r x-1$, the sole C. elegans ortholog of neurexin, disrupts these dendritic spines and impairs proper localization of cholinergic receptor clusters to postsynaptic sites on GABAergic dendrites (Philbrook et al., 2018). Here we define the order and timing of pre- versus postsynaptic development at spine-associated synapses and elucidate the role of neurexin in the coordination of these processes in vivo. We find that clusters of both presynaptic proteins and postsynaptic receptors are clearly visible prior to spine outgrowth. In the absence of neurexin, immature spines and receptor clusters form initially but then disappear, indicating that neurexin is required for synapse stabilization and maturation rather than synaptogenesis. These maintenance and maturation processes are supported by kinesin-3/UNC-104 motor dependent transport of NRX-1 to presynaptic terminals. Together, our results suggest that 
bioRxiv preprint doi: https://doi.org/10.1101/2021.05.11.443328; this version posted June 24, 2021. The copyright holder for this preprint (which was not certified by peer review) is the author/funder. All rights reserved. No reuse allowed without permission.

axonal delivery of neurexin to sites of synapse formation is critical for stabilization of mature postsynaptic structures. 


\section{Results}

We initially explored morphological features of mature spines using 3D rendering of spines from confocal imaging. We found that a majority of DD spines could be categorized into four morphological classes (percentage $\pm S D$ ): mushroom $(5 \pm 1 \%)$, branched $(5 \pm 1 \%)$, stubby (74 $\pm 3 \%)$, and thin (15 $\pm 1 \%)$ (Figure 1B). The morphological diversity we observed by light microscopy was also evident in ultrastructural studies using serial electron microscopy, as noted previously (Figure S1.1) (J. G. White et al., 1976) (J. G. White et al., 1986) (Cuentas-Condori et al., 2019). We noted clear morphological variation across individual spines, though spine profiles occasionally spanned several sections. Additionally, clusters of small synaptic vesicles were evident in neurites (of cholinergic VA/VB neurons, presumably) immediately adjacent to the tips of DD spines, indicating putative sites of synaptic input.

To explore how the neuronal cytoskeleton may contribute to spine morphology, we examined the localization of F-actin and tubulin in DD dendrites using DD neuron-specific expression GFP::UtrCH (GFP fused to the Utrophin calponin homology domain) (Chia et al., 2014) and TBA-1::GFP (GFP-tagged $\alpha$-tubulin) (Yan et al., 2013) respectively. We found that the two markers largely occupy distinct territories. Coexpression studies showed that F-actin is strongly enriched within dendritic spines (Figure 1C-E), while tubulin occupies the dendritic shaft (Figure 1F, G) with more variable localization to the spine base (Figure S1.2). Previous findings for vertebrate neurons indicate a similar segregation of actin and tubulin to the spines and shaft of the dendrite respectively (Gu et al., 2008; Hu et al., 2008; Jaworski et al., 2009).

DD spines are positioned immediately adjacent to the axon terminals of cholinergic neurons (Figure S1.1) (Cuentas-Condori et al., 2019; J. G. White et al., 1976, 1986). We previously showed stimulation of cholinergic motor neurons elicited $\mathrm{Ca}^{2+}$ responses in GABAergic motor neurons (Philbrook et al., 2018). To confirm that spines on DD GABAergic 
motor neurons represent sites of functional synaptic contacts, we asked whether stimulation of presynaptic cholinergic neurons was sufficient to elicit calcium responses in DD dendrites. We performed in vivo calcium imaging of evoked responses in spines using combined expression of a membrane-associated GCaMP6f calcium sensor in DD GABAergic neurons and a red shifted channelrhodopsin, Chrimson, in cholinergic neurons (Philbrook et al., 2018) (Figure 1H-J, S1.3). Following 5 seconds of baseline recording ( $488 \mathrm{~nm}, 100 \mathrm{~ms}$ exposure), we measured calcium responses to presynaptic depolarization $\left(5 \mathrm{~s}, 625 \mathrm{~nm}, 30 \mathrm{~mW} / \mathrm{cm}^{2}\right.$ ) (Figure 1I). We noted significant fluorescence increases in spines that were timed with the onset of stimulation and were not present in the absence of retinal. These typically occurred simultaneously across multiple spines and returned to baseline within $5 \mathrm{~s}$ following stimulation. Importantly, mutation of the cholinergic vesicular transporter/VAChT unc-17, reduced evoked calcium responses by $81 \%$, indicating the $\mathrm{Ca}^{2+}$ responses we observed in dendrites were dependent on presynaptic acetylcholine release (Figure 1I, J). Our findings are consistent with those of another recent study (Cuentas-Condori et al., 2019) indicating that spines on DD GABAergic neurons are primary sites of synaptic input.

Mitochondria and other organelles are often localized near postsynaptic specializations in order to sustain synaptic function (Vos et al., 2010) (Karagas \& Venkatachalam, 2019). To examine the distribution of organelles within DD dendrites and spines, we used fluorescent reporters labeling Golgi (AMAN-2::GFP) (Edwards et al., 2013), mitochondria (Pre-Su9::GFP) (Sarasija \& Norman, 2015), and endoplasmic reticulum (ER) (RFP::TRAM-1) (Rolls et al., 2002) (Edwards et al., 2013) (Figure S1.4). We found that Golgi is exclusively labeled in the DD cell bodies (Figure S1.4A). In contrast, mitochondria and rough ER are dispersed throughout the main dendritic shaft and near the base of spines (Figure S1.4B-E), perhaps suggesting involvement in spine function (Perez-Alvarez et al., 2020) (Z. Li et al., 2004). Consistent with our 
light microscopy observations, we also observed mitochondria near spines in the main dendritic process by electron microscopy (Figure S1.1B-D).

\section{The development of synapses at DD GABAergic spines}

Using the synapses at DD spines as a model, we next investigated the relative timing of pre- and postsynaptic events during synapse formation. C. elegans progress through four larval stages of development (L1-L4) prior to adulthood. DD neurons undergo a well-characterized program of synaptic remodeling such that the mature circuit organization is established during the transition from L1 to L2 stage (Witvliet et al., 2021) (J. G. White et al., 1978). The formation of new synaptic connections between postembryonic born cholinergic neurons and the ventral dendrites of DD GABAergic neurons offers a window to investigate de novo formation of neuron-neuron synapses. We focused on understanding the time course of spine formation relative to 2 key events in synaptogenesis: 1) the formation of presynaptic release sites, and 2) the clustering of postsynaptic receptors and F-actin (Figure 2).

The appearance of newly born ventral cholinergic motor neurons progressed anteriorly to posteriorly starting roughly 16 hours after hatch, consistent with the birth and integration of cholinergic neurons during this time frame (Figure S2.1) (Kurup \& Jin, 2016). We analyzed the distribution of pre- and postsynaptic markers at timepoints before (12 hours after hatch), during $(16,18,20$ hours after hatch) and after (24, 32 hours after hatch, L4 stage/42-50 hours) DD synaptic remodeling (Figures 2, S2.1, and S2.2). We first assessed the formation of the active zone and presynaptic specialization by analyzing clustering of the Piccolo-like active zone protein, CLA-1, (GFP::CLA-1e) (Xuan et al., 2017) and the synaptic vesicle-associated protein synaptobrevin/SNB-1 (SNB-1::GFP) (Figure 2A, D-E). Overall, we found CLA-1 is more discretely localized to putative synapses than SNB-1, both at earlier timepoints and throughout development, consistent with its specific localization to active zones. Immediately prior to 
remodeling ( 12 hours after hatch), we did not observe significant localization of either presynaptic CLA-1 or SNB-1 adjacent to the DD process. Shortly after (16 hours after hatch), we noted the initial appearance of SNB-1- and CLA-1-associated fluorescence in presynaptic cholinergic processes. Over the next 4 hours (20 hours after hatch) individual CLA-1 clusters were more clearly distinguishable, suggesting CLA-1 association with developing active zone structures (Figure 2A, D, L). Synaptic vesicle fluorescence was initially diffuse at 16 hours after hatch and became more clearly organized into discrete puncta over a similar time course to CLA-1 (Figure 2A, E, L). We did not observe the emergence of DD dendritic spines until 24-32 hours after hatch, well after initial active zone formation and recruitment of synaptic vesicles (Figures 2A, C-E). Spines continued to mature through L4 stage, increasing in both length and number (Figure S2.2).

We next examined the clustering of postsynaptic acetylcholine receptors in DD dendrites (ACR-12::GFP). Prior to remodeling (12 hours after hatch), we did not observe detectable levels of cholinergic receptors in the DD process. Surprisingly, we noted immature receptor clusters faintly visible in the dendritic shaft by 16 hours after hatch, well prior to the emergence of dendritic spines (Figure 2B, F, L). These receptor clusters increased in number and redistributed towards the tips of growing spines by 32 hours (Figure 2B, F, L). We found a similar developmental trend using DD neuron-specific labeling of the LEV-10 transmembrane auxiliary protein (Gally et al., 2004), previously shown to concentrate in spines (He et al., 2019) (Figure S2.3). By L4 stage, receptor and LEV-10 clusters are clearly visible at the tips of mature spines. Our analysis indicates that the initial stages of development of postsynaptic (AChR and LEV-10 clusters) structures occur prior to spine outgrowth, raising questions about how these initial processes may be regulated. 
To begin to address this question, we analyzed the distribution of F-actin in dendrites of the DD neurons during synapse formation (Figure 2B, G-L). We noted that clusters of F-actin were evident in the ventral DD process prior to the completion of synaptic remodeling (12 hours after hatch), prior to presynaptic CLA-1 and synaptic vesicle accumulation, and before the clustering of postsynaptic receptors. Dendritic F-actin-based structures became more abundant coincident with increases in the number of presynaptic CLA-1 and postsynaptic receptor clusters. To investigate this process in real-time, we used live imaging to examine the dynamics of postsynaptic F-actin (GFP::UtrCH) in the developing DD dendrite. We found that F-actin is highly dynamic during early developmental stages (16-20 hours after hatch) compared to L4 stage where the circuit has completed maturation (Figure S2.4, Videos 1 and 2). In young animals, GFP::UtrCH clusters often shuttled out of the cell body to the main dendritic process, perhaps indicating redistribution of F-actin to sites of postsynaptic assembly. By 24 hours after hatch, we observed clear co-localization of F-actin with newly formed AChR clusters in the dendritic shaft. By L4 stage, AChR clusters are stably sequestered at the tips of spines, while Factin occupies the spine head and neck (Figure $2 \mathbf{H}-\mathbf{K}$ ).

\section{Dendritic spines form in the absence of presynaptic activity}

Our above analysis indicated that the localization of synaptic vesicles and active zone proteins, such as CLA-1, occur prior to spine outgrowth. In the rodent brain, spine morphogenesis is clearly regulated by presynaptic activity (De Roo et al., 2008; Engert \& Bonhoeffer, 1999; Sala \& Segal, 2014; Saneyoshi et al., 2010), but recent evidence suggests initial spine outgrowth may proceed independently of synaptic activity (Lu et al., 2013) (Sigler et al., 2017) (Sando et al., 2017). We therefore next asked whether presynaptic cholinergic activity is important for the formation of DD dendritic spines. To address this question, we analyzed the number of dendritic spines (Pflp-13::mCherry or Pflp-13::myrGFP) and cholinergic receptors 
(Pflp-13::ACR-12::GFP) at L4 stage in strains carrying mutations that affect various aspects of synaptic function and neuronal excitability (Table 1, Figure S3.1).

Surprisingly, the abundance of spines and receptor clusters at L4 stage were not significantly affected by disruption of ACh synthesis in cholinergic motor neurons (mutation of the vesicular acetylcholine transporter unc-17) or by a strong reduction in synaptic vesicle exocytosis (mutation of the syntaxin binding protein unc-18). Similarly, loss-of-function mutations in genes required for dense core vesicle release (CAPS/unc-31) and $\mathrm{Ca}^{2+}$ signaling (VGCC/unc-2) did not significantly reduce the number of spines or receptor clusters in the mature circuit (Table 1, Figure S3.1). Our data indicate that severe alterations in synaptic activity only weakly affect the abundance of L4 stage dendritic spines. However, we cannot exclude the possibility of developmental delays in spine formation (Cuentas-Condori et al., 2019). Notably, many of the mutations we examined significantly altered spine length and the size of receptor clusters (Table 1), suggesting that activity may influence other aspects of spine morphology and postsynaptic structure. Together, our findings point toward a model where initial spine formation and postsynaptic development proceed without a strong requirement for synaptic activity, while subsequent spine morphogenesis is more clearly affected.

\section{Presynaptic NRX-1 stabilizes postsynaptic components}

In previous work, we found that mutations in $n r x-1$, the sole $C$. elegans ortholog of neurexin, eliminate DD dendritic spines and disrupt cholinergic receptor clusters in mature $C$. elegans (Philbrook et al., 2018), indicating alternate activity-independent mechanisms may be critical for spine formation. Consistent with these findings, we found that mutation of $n r x-1$ disrupted dendritic calcium responses evoked by presynaptic stimulation of cholinergic motor neurons (Figure S3.2). To assess involvement of presynaptic NRX-1 in the establishment of spines, we asked when NRX-1 first localizes to presynaptic terminals of cholinergic motor 
neurons. Axonal clusters of NRX-1::GFP are present by 16 hours after hatch (Figure 3A, B), approximately the same time at which assemblies of the presynaptic scaffold CLA-1 become visible (Figure 2). Diffuse synaptic vesicle material is also visible at this time (Figure 2) but has yet to be organized into discrete clusters.

The early arrival of NRX-1 to synaptic terminals could indicate a role in either initial synapse formation or subsequent stabilization and maturation. To distinguish between these possibilities, we analyzed the development of dendritic spines and cholinergic receptor clusters in $n r x-1$ (wy778) null mutants. We quantified spine and AChR cluster number over a similar time course as previously completed for wild type. Surprisingly, we noted that immature spines and receptor clusters were evident in $n r x-1$ mutants during early development, albeit at slightly reduced density relative to wild type (Figures $3 \mathrm{C}-\mathrm{F}, \mathbf{S 3 . 3}$ ). The density of spines in $n r x-1$ mutants increased significantly over the next several hours until 24 hours after hatch (Figure 3C,D, S3.3). However, after this time, spine density decreased dramatically such that $n r x-1$ DD dendrites were almost completely devoid of spines by L4 stage (Philbrook et al., 2018). Measurements of spine formation and disassembly in live imaging studies of wild type and $n r x-1$ mutants offered further support for this conclusion. Mature wild type spines (L4) were remarkably stable over 1-2 hours of recording, but were more dynamic in the developing circuit (16-20 hrs after hatch) (Figure S3.4, Videos 3-5). Developing spines in $n r x-1$ mutants were also highly dynamic. Notably, nrx-1 mutant spines exhibited heightened dynamics into later stages of development compared with wild type spines. For example, at 21-24 hours after hatch, almost $90 \%$ of wild type spines were stable over the recording period, while less than $50 \%$ of $n r x-1$ mutant spines remained stable.

Receptor clustering followed a similar trend to spine formation. The number and fluorescence intensity of ACR-12 clusters at 16 hours after hatch in $n r x-1$ (wy778) animals (6.8 \pm 
0.8 clusters $/ 25 \mu \mathrm{m})$ were similar to wild type (5.6 \pm 0.7 clusters/25 $\mu \mathrm{m})$ (Figure 3E, F, S3.3). Within a few hours however, ACR-12 cluster number and intensity decreased significantly in nrx-1 mutant dendrites, and remained low throughout the remainder of development (Figure 3E, F, S3.3). Remarkably, we found that the organization of dendritic F-actin was similarly affected by $n r x-1$ deletion. GFP::UtrCH was arranged similarly across $n r x-1$ mutants and wild type in early development (16 hrs after hatch), positioned in discrete clusters along the main dendritic process (Figure 3G). By L4 stage, wild type F-actin was almost entirely associated with dendritic spines. In contrast, GFP::UtrCH was diffusely localized along the length of L4 stage nrx-1 mutant dendrites (Figure 3G). Taken together, our results indicate that presynaptic NRX-1 is dispensable for the earliest stages of postsynaptic assembly and spine formation, but is critical for stabilizing dendritic spines, F-actin assemblies and AChR clusters, and promoting their maturation.

\section{Kinesin-3/UNC-104 transports presynaptic neurexin to cholinergic terminals in order to stabilize postsynaptic components}

To investigate this model further, we sought to determine how presynaptic neurexin is transported to active zones in vivo. Prior work showed that both synaptic vesicles and CLA-1 depend on the Kinesin-3 motor UNC-104 for their delivery to synapses (Hall \& Hedgecock, 1991) (Xuan et al., 2017). In particular, disruption of unc-104 causes an accumulation of synaptic vesicles within neuronal somas and a corresponding loss of synaptic vesicles within axons (Hall \& Hedgecock, 1991) (Figure S4.1). unc-104(e1265) carries a D1497N mutation in the $\mathrm{PH}$ domain of UNC-104 that impairs cargo binding and leads to a severe reduction in axonal UNC-104 abundance (Kumar et al., 2010). We found that NRX-1 endogenously tagged with GFP localized to puncta in neuronal processes of the nerve ring and nerve cords of wild type animals (Figure S4.2), but was strikingly decreased in the nerve cords of unc-104(e1265) mutants (Figures 4A,C, S4.3). A striking deficit in axonal NRX-1::GFP localization was also 
evident with specific cholinergic expression of NRX-1::GFP in unc-104 mutants (Figures 4B,C, S4.3). By contrast, NRX-1::GFP fluorescence was significantly increased (5.4-fold) in unc-104 mutant cholinergic somas compared to wild type (Figure S4.3). Consistent with UNC-104 involvement in cholinergic NRX-1 trafficking, we found that UNC-104 and NRX-1 are partially colocalized in cholinergic axons (Figure S4.4). Accumulation of NRX-1 in cholinergic cell bodies, coupled with a decrease in axons, indicates a failure of NRX-1 transport in the absence of functional UNC-104. Cholinergic-specific expression of wild type unc-104 in unc-104 mutants restored axonal NRX-1::GFP localization, indicating a cell autonomous requirement (Figure 4B,C). In contrast, mutation of the Kinesin-1 motor unc-116 did not produce significant accumulation of NRX-1::GFP in cholinergic somas and caused comparatively modest decreases in axonal NRX-1::GFP, demonstrating preferential involvement of Kinesin-3 for NRX1 transport (Figure S4.5). Live imaging studies offered additional evidence that synaptic vesicle and NRX-1 trafficking may share a common dependence on UNC-104. We found that NRX1::GFP trafficking events occurred with similar anterograde and retrograde velocities to SNB1::GFP (labeling synaptic vesicles) trafficking events, though less frequent (Figure S4.6, Videos 6 and 7). Prior studies have noted that active zone protein trafficking events occur at reduced frequency compared with synaptic vesicle trafficking (Lipton et al., 2018). Together, our analyses point to a specific requirement for the UNC-104/KIF1A motor in delivery of NRX-1 to presynaptic terminals.

The density of spines and AChR clusters were also severely reduced in L4 stage unc104(e1265) mutants (Figure 4D-F), similar to $n r x-1$ mutants (Figure 4G). These effects were rescued by either native or cholinergic expression of wild type unc-104 in unc-104(e1265) mutants, but not by GABA- or muscle-specific unc-104 expression (Figure 4D-F), demonstrating that UNC-104-mediated transport is critical in presynaptic cholinergic axons for postsynaptic spine development and receptor localization. 
We next asked how a failure in delivery of $A Z$ components may contribute to the severe reductions in spines and receptor clusters we observed in unc-104 mutants. In addition to CLA1 (Xuan et al., 2017), transport of both ELKS-1/ELKS/CAST and UNC-10/RIM were severely disrupted by mutation of unc-104, indicating that Kinesin-3 mediated transport is key for the synaptic delivery and assembly of several active zone constituents in these neurons (Figure 4H-J). Remarkably, we found that only mutation of $n r x-1$ produced significant decreases in dendritic spines (Figure 4G), demonstrating that presynaptic ELKS, UNC-10 and CLA-1 are dispensable for postsynaptic development. Our results demonstrate a specific requirement for NRX-1 in the stabilization and maturation of postsynaptic structures and provide evidence that a failure in synaptic delivery of NRX-1 is a primary causal factor in the postsynaptic structural defects of unc-104 mutants.

\section{The stabilization of mature dendritic spines requires ongoing synaptic delivery of NRX-1}

Our results suggest UNC-104 mediated transport positions NRX-1 at the presynaptic terminal in the early stages of synapse formation where it acts to stabilize growing postsynaptic structures, including spines and receptor clusters. We next sought to address whether there is a requirement for presynaptic UNC-104-dependent transport in the stabilization of mature spines. To address this question, we used the previously characterized temperature-sensitive allele, unc-104(ce782). unc-104(ce782) animals carry a G105E missense mutation in the motor domain of UNC-104 (Edwards et al., 2015). When grown at the permissive temperature $\left(13.5^{\circ} \mathrm{C}\right)$, unc-104(ce782) axonal synaptic vesicle abundance and animal motility are modestly reduced compared to wild type. In contrast, synaptic vesicles are completely absent from unc104 (ce 782) axons following growth at restrictive temperature $\left(20-25^{\circ} \mathrm{C}\right)$, and animal motility is severely compromised within 12 hours of a shift to $23^{\circ} \mathrm{C}$ (Edwards et al., 2015). We raised unc104(ce782) animals to L4 stage at the permissive temperature $\left(13.5^{\circ} \mathrm{C}\right)$ (Figure 5). We then 
shifted L4 stage animals to the restrictive temperature $\left(25^{\circ} \mathrm{C}\right)$ for $16-20 \mathrm{hrs}$, and quantified NRX$1::$ GFP localization and spine density immediately following this shift. Axonal NRX-1::GFP clusters were strikingly decreased in unc-104(ce782) mutants subjected to the temperature shift compared with control animals subjected to the same shift or unc-104(ce782) mutants raised continuously at the permissive temperature (Figure 5B,C). unc-104(ce782) mutants grown at the permissive temperature had a slightly reduced number of spines overall compared with wild type (Figure 5E). A shift to the restrictive temperature at L4 stage produced a striking reduction in spine density for unc-104(ce782) mutants (Figure 5D, E), but not for wild type. We obtained similar results for unc-104(ce782) mutants using an earlier shift to the restrictive temperature (at L3 stage) (Figure S5.1). Taken together, our findings indicate an ongoing requirement for UNC104 transport that extends well beyond the period of initial synapse formation and spine outgrowth. Among the UNC-104 cargoes we investigated, only deletion of $n r x-1$ produces a significant reduction in spine density at $L 4$ stage. We therefore propose a model where ongoing UNC-104 delivery of presynaptic NRX-1 is critical for postsynaptic maturation and maintenance of mature spines. 


\section{Discussion}

\section{C. elegans GABAergic motor neurons are decorated with functional dendritic spines}

Dendritic spines are known to act as specialized sites for compartmentalizing neurotransmission and are widely observed across various classes of neurons in both vertebrates and invertebrates (Tonnesen \& Nagerl, 2016). Though suggested by prior EM studies (J. G. White et al., 1976) (J. G. White et al., 1986), the presence of functional dendritic spines on C. elegans neurons had not been fully appreciated until recently (Philbrook et al., 2018) (Oliver et al., 2018) (Cuentas-Condori et al., 2019). Here we examined the developmental trajectory of postsynaptic structures (dendritic spines and cholinergic receptor clusters) located on GABAergic motor neurons relative to presynaptic release sites and showed that the stabilization of growing dendritic spines requires presynaptic NRX-1/neurexin.

Neural circuit organization and performance is dictated by the locations of synaptic connections and the identities of the interconnected neurons. Elucidation of the processes governing the construction of synapses and their coordination across presynaptic axons and postsynaptic dendrites are therefore fundamental for understanding circuit assembly and function. However, relatively few studies have monitored in real-time the coordinated assembly of pre- and postsynaptic specializations in vivo. Our work tackled this important question, taking advantage of the GABAergic DD spine model and the cellular precision offered by the $C$. elegans motor circuitry. We find that these dendritic spines share many of the hallmark features of vertebrate dendritic spines, but also have some key differences. (1) C. elegans spines have morphological similarities with mammalian spines and can be classified into comparable morphological categories. (2) F-actin is localized to spines while tubulin is mainly present in the dendritic shaft. F-actin is a major component of mammalian dendritic spines and is important for spine structural dynamics (reviewed in (Borovac et al., 2018)). (3) Mitochondria and rough 
endoplasmic reticulum are positioned near spines, either in the spine neck or at the base of spines in the dendritic shaft. Dendritic organelles contribute to key processes involved in spine formation and spine metabolism (Liu \& Shio, 2008). Ribosomes and mitochondria positioned near or within dendritic spines may aid in modifying dendritic spine activity, local protein synthesis, and act as synaptic ATP and $\mathrm{Ca}^{2+}$ sources (Aakalu et al., 2001; Chirillo et al., 2019; Z. Li et al., 2004; Steward \& Levy, 1982). (4) Presynaptic stimulation elicits calcium responses in spines on GABAergic dendrites. DD spines are located opposite cholinergic release sites and calcium responses are eliminated by mutation of the vesicular acetylcholine transporter. (5) Mature C. elegans spines display limited overt activity-dependent dynamics in wild type animals and appear comparatively stable relative to spines in rodents. This raises the important question of what is the functional role of spines in GABAergic neurons? Prior studies have provided evidence for compartmentalized calcium signals in C. elegans neurons (Hendricks et al., 2012). Therefore, one intriguing possibility is that, similar to the situation in mammals, spines serve to compartmentalize calcium and perhaps other biochemical signals in GABAergic neurons. Spines may also be required in order to achieve the unusual dyadic arrangement of synapses in the ventral nerve cord, where presynaptic specializations of cholinergic motor neurons are positioned for transmission onto both GABA motor neuron and muscle postsynaptic partners (Hall \& Russell, 1991). In this case, GABAergic spines may have developed to intercept cholinergic release sites onto muscles, as suggested previously (J. White, 2018).

We found that F-actin is compartmentalized to discrete regions within the dendritic shafts of DD neurons very early in the development of the circuit (prior to the appearance of spines) and later becomes exclusively localized to dendritic spines. F-actin labeling of spines is apparent as soon as they are detectable, suggesting that F-actin assemblies may participate in the earliest stages of spine development. Prior to remodeling of DD neurons, sites of GABA release are located on the ventral processes of DD neurons. As F-actin based structures are 
typically associated with release sites (Chia et al., 2012) (Meng et al., 2015), our finding that Factin is clustered in ventral DD processes prior to remodeling may suggest that F-actin initially associated with release sites persists in the DD neurite through remodeling and is then repurposed for postsynaptic development, perhaps acting as a landmark or actin-based mechanism for stimulating postsynaptic maturation.

Notably, we also observed punctate cholinergic receptor fluorescence in GABAergic dendrites several hours prior to the formation of dendritic spines. These immature receptor clusters colocalize with dendritic F-actin assemblies, suggesting that receptors are trafficked into dendrites and positioned with F-actin to rapidly populate growing spines. Indeed, we observed that receptor clusters are visible in growing spines as soon as spines can be clearly resolved. This is consistent with recent findings from time-lapse 2-photon imaging of hippocampal organotypic slice cultures showing that receptor accumulation in spines occurs concurrently with spine outgrowth (Zito et al., 2009). Similarly, we found that synaptic material accumulates in presynaptic axons prior to the emergence of spines from dendritic processes. In particular, initial accumulations of the presynaptic scaffold CLA-1 and the synaptic vesicle marker synaptobrevin/SNB-1 were visible in the axon with a similar time-course to the appearance of dendritic cholinergic receptor clusters. Presynaptic material became more clearly localized to discrete puncta over the next 4-6 hours, occurring roughly coincident with receptor accumulation at the tips of growing spines. Notably, our findings do not support a strong requirement for presynaptic neurotransmitter release in spine formation. The abundance of spines was either mildly affected or unaffected across several mutant strains with severely impaired presynaptic function.

\section{Transsynaptic NRX-1/Neurexin signaling stabilizes dendritic F-actin assemblies to promote postsynaptic maturation}


Numerous studies across a variety of model systems have examined roles for neurexins in synapse development and function. However, no single consensus view of neurexin function has emerged. Instead, functions for neurexins at specific synapses are thought to be dictated by cellular and molecular context. Mammalian genomes encode three Nrxn genes that can give rise to $\alpha-, \beta-$, and $\gamma$-Nrxn isoforms. These isoforms share common intracellular and transmembrane regions but differ in their extracellular domains. $\alpha-N r x n$ has six extracellular LNS domains interleaved with three EGF-like repeats. $\beta$-Nrxn has only one LNS domain, while $\gamma$-Nrxn lacks all identifiable extracellular domains. Conditional triple knockout of mouse neurexins indicated synapse loss in parvalbumin-positive prefrontal cortex interneurons, but not somatostatin-positive interneurons (L. Y. Chen et al., 2017). At mouse calyx synapses, conditional triple neurexin panneuronal knockout indicated a role in active zone organization and $\mathrm{Ca}^{2+}$ and $\mathrm{BK}$ channel regulation, but no requirement in synapse formation or maintenance (Luo et al., 2020). The multi-faceted roles for neurexins at vertebrate synapses likely emerge as a consequence of the many neurexin isoforms that are generated by alternative splicing, the complexity of their cellular expression, and the potential for these isoforms to selectively interact with distinct postsynaptic partners.

The $C$. elegans genome encodes a single neurexin gene, $n r x-1$, which generates long $\alpha$ and short $\gamma$ isoforms (Haklai-Topper et al., 2011). As is the case in other systems, C. elegans $\mathrm{NRX}-1$ has roles in presynaptic organization, for example in calcium channel clustering at the active zone (Kurshan et al., 2018). Interestingly, the short $\gamma$-NRX-1 isoform that lacks identifiable ectodomains performs these roles (worms do not encode $\beta-N R X-1$ ), but even in the absence of both isoforms, a level of presynaptic functionality is maintained, as indicated by the presence of evoked responses in muscle cells following presynaptic motor neuron stimulation (Philbrook et al., 2018). Our prior studies pointed toward the importance of NRX-1 at synapses between 
cholinergic and GABAergic motor neurons (Philbrook et al., 2018). Surprisingly, our time course studies here revealed that initial spine outgrowth and AChR clustering occurs normally in $n r x-1$ mutants. However, spines and AChRs in nrx-1 mutants become destabilized within hours and disperse, such that GABAergic dendrites are almost completely devoid of these postsynaptic components by L4 stage. Dendritic F-actin assemblies, that normally show discrete localization to spines, are also disorganized in nrx-1 mutants, and are diffusely distributed throughout GABAergic dendritic processes in $n r x-1$ mutants. We propose a model where presynaptic NRX1 is required for the stabilization of dendritic spines and other postsynaptic structures in GABAergic dendrites. We suggest that presynaptic NRX-1 directs the organization of dendritic F-actin to stimulate maturation and stabilization of spines and postsynaptic receptor clusters. A similar form of F-actin based reorganization is a key step in presynaptic differentiation (Chia et al., 2012) (Chia et al., 2014). Our findings contrast with previous culture studies that implicated neurexin in postsynaptic induction (Graf et al., 2004).

Our work has parallels with recent studies in Xenopus (S. X. Chen et al., 2010) and Drosophila (Constance et al., 2018). In embryonic Xenopus brain, presynaptic $\beta$-neurexin stabilizes dendritic filopodia through an adhesive partnership with neuroligin to direct the development of dendritic arbors (S. X. Chen et al., 2010). Similarly, during fly metamorphosis, neurexin/neuroligin-based adhesion promotes the growth of neurite branches independently of synaptic activity (Constance et al., 2018). In our studies, NRX-1 stabilization of dendritic spines occurs independently of synaptic activity and also without involvement of neuroligin (Philbrook et al., 2018), suggesting interaction with an alternate binding partner that to date remains unidentified.

\section{UNC-104/Kinesin 3-mediated synaptic delivery of NRX-1/Neurexin is an essential early step required for synapse maturation and stabilization}


Efficient trafficking of synaptic and active zone cargoes to presynaptic terminals is essential for synapse formation and neuronal communication. Long-range axonal transport of synaptic vesicles and active zone proteins is carried out by anterograde kinesin and retrograde dynein motors. However, significant questions remain about which synaptic proteins are trafficked together and their dependence on specific motors. We found a specific requirement for the kinesin-3 UNC-104/KIF1A motor to deliver NRX-1 to presynaptic terminals. The anterograde transport of synaptic and dense core vesicles are also strongly dependent upon UNC-104 (Hall \& Hedgecock, 1991) (Zahn et al., 2004). In contrast, several studies suggest that other AZ proteins exhibit weaker requirements for UNC-104 in their delivery. For example, ELKS-1 and other AZ proteins localize to C. elegans HSN synapses in an UNC-104independent manner (Patel et al., 2006). Similarly, UNC-10/RIM is properly concentrated in nerve ring processes of unc-104(e1265) mutants (Koushika et al., 2001). Here, we found that NRX-1, UNC-10/RIM, ELKS-1/ELKS and CLA-1/piccolo all require UNC-104 for anterograde transport to cholinergic motor neuron synapses, demonstrating cell-specific differences in the dependence on UNC-104.

Prior studies of rodent cultured neurons also suggested a requirement for the Kinesin-3 motor in neurexin transport, indicating that mechanisms for NRX-1 delivery to synapses are conserved (Neupert et al., 2015). We found that dendritic spines collapse and AChR clusters disperse when UNC-104 delivery of synaptic cargoes is disrupted. Of the UNC-104 cargoes we analyzed, only mutation of $n r x-1$ produced significant disruption of dendritic spines. We therefore propose that NRX-1 is a key UNC-104 cargo required for spine stabilization. Our live imaging points to similar rates of transport for synaptic vesicles and NRX-1. However, it remains unclear whether NRX-1 is delivered as a component of synaptic vesicles or may be segregated into a distinct vesicular population, such as synaptic vesicle protein transport vesicles (STVs). 
Our findings that mutations which severely impair synaptic vesicle fusion do not affect spine density point toward the latter possibility.

The prior identification of a temperature-sensitive allele of unc-104 allowed us to explore the temporal requirements for UNC-104 delivery of NRX-1. Importantly, we found that UNC-104 delivery was not solely required during early stages of synapse maturation but was critical throughout the developmental progression of the circuit toward maturity. These findings argue that delivery of presynaptic NRX-1 is required to maintain postsynaptic structure long after circuit assembly is complete. This raises interesting questions about the relationship between NRX-1 transport and synapse stability, perhaps suggesting that alterations in the rate of axonal NRX-1 transport may directly impact synaptic connectivity in mature animals. More broadly, our studies provide a new view of adhesive mechanisms in circuit connectivity and highlight a novel role for neurexin in the stabilization of mature synapses and dendritic spines. 


\section{Materials and Methods}

\section{Strains}

All strains are N2 Bristol strain derivatives (wild type) and were maintained at room temperature $\left(20-24^{\circ} \mathrm{C}\right)$ on nematode growth media plates (NGM) seeded with E. coli strain OP50.

Transgenic strains were obtained by microinjection to achieve transformation (Mello et al., 1991) and identified using co-injection markers. Integrated lines were produced with X-ray irradiation and outcrossed to wild type/N2 Bristol eight times. Only hermaphrodites (L1-L4, young adults) were used in this study. A complete list of all strains used in this study is found in Supplemental

File 1. Worms used for time course studies were staged by transferring freshly hatched larvae to seeded OP50 plates and transferring to $25^{\circ} \mathrm{C}$ (time point 0 ).

\section{Molecular Biology}

Plasmids were constructed using the two-slot Gateway Cloning system (Invitrogen) and confirmed by restriction digest and sequencing.

Utrophin/F-actin reporter. Sequence coding for mCherry was amplified from pDest-16 (and ligated into Agel-HF/BspEl-digested PNYL183 (Pflp-13::GFP::UtrCH, gift from Dong Yan's laboratory) to make pCL87 (Pflp-13::mCherry::UtrCH).

TBA-1/tubulin reporter. Sequence coding for GFP::TBA-1 was amplified from plasmid pYJ128 (gift from Kang Shen laboratory) and ligated into a destination vector to create pDest-173.

pDest-173 was recombined with pENTR-5'-flp-13 to generate pD061 (Pflp-13::GFP::TBA-1).

Golgi apparatus reporter. pDest-161 (AMAN-2::GFP) was recombined with pENTR-3'-flp-13 to generate pDO47 (Pflp-13::AMAN-2::GFP). pDest-161 was created by HindIII-HF digestion of Pgpa-4::AMAN::GFP (gift from Gert Jansen's laboratory) and ligated with a destination vector. Mitochondria reporter. pre-su9::GFP was isolated from Xmal/Sphl-HF digested pDM1389 (gift from A.V. Maricq lab) and ligated into a destination vector to create pDest-91 (pre-su9::GFP). pDest-91 was recombined with pENTR-5'-flp-13 to generate pAP102 (Pflp-13::pre-Su9::GFP). 
ER reporter. Tag-RFP::TRAM-1 was amplified from plasmid pCT27 (gift from A.V. Maricq laboratory) and ligated to a Kpnl-HF/NgoMIV digested destination vector to generate pDest-169. pDest-169 was recombined with pENTR-3'-flp-13 to generate pDO59 (Pflp-13::RFP::TRAM-1). Presynaptic vesicle reporter. acr-5 promoter was amplified from plasmid pDM806 and ligated into pENTR-D-TOPO to generate pENTR-40. pENTR-40 was then recombined with pDest-5 to generate pAP264 (Pacr-5::SNB-1::GFP).

B-type specific motor neuron reporter. pDO45 (Pacr-5::GFP) was created by recombining pDest-94 (GFP) and pENTR-40 (acr-5 promoter).

myrGCamp6f. myrGCaMP6f was amplified from plasmid pDest-164 and ligated into a destination vector to create pDest-180. pDest-180 was recombined with pENTR3'-flp-13 to create pDO69 (Pflp-13::SL2::myrGCamp6f).

CLA-1 reporter. 3XnovoGFP::CLA-1e was isolated from Sphl-HF/Agel-HF digested pKP85 (gift from Peri Kurshan) and ligated with a destination vector to create pDest-307 pDest-307 was recombined with pENTR-3'-unc-17 $\beta$ to generate pSR58 (Punc-17 $\beta: 3$ XnovoGFP::CLA-1e cDNA).

unc-104 rescue constructs. UNC-104cDNA::mCherry was isolated from Sphl-HF/Nhel-HF digested pLL48 (gift from Kang Shen lab) and ligated with a destination vector to make pDest308. pDest-308 was recombined with pENTR-unc-17 $\beta$ to generate $\mathrm{pDO} 126$ (Punc-17 $\beta$ :UNC104cDNA::mCherry), pENTR-3'-unc-47 to generate pD0134 (Punc-47::UNC104cDNA::mCherry), and pENTR-3'-myo-3 to generate pD0136 (Pmyo-3::UNC104cDNA::mCherry).

Punc-17 $\beta:: N R X-1::$ GFP. pAP112 was generated by recombining pENTR-3'-unc-17 $\beta$ with pDest99 (NRX-1::GFP). 
ELKS-1::mCherry. mCherry::ELKS-1cDNA was isolated from Sphl-HF/Ascl digested pK043 (gift from Peri Kurshan laboratory) and ligated with a destination vector to create pDest-325. pDest325 was recombined with pENTR-unc-129 to generate pDO156 (Punc-129::ELKS-1::mCherry). UNC-10::GFP. UNC-10::GFP was isolated from BamHI-HF/Sbfl digested pRIM3 (gift from Michael Nonet laboratory) and ligated with a destination vector to generate pDest-304. pDest304 was recombined with pENTR-3'-unc-129 to generate pDO128 (Punc-129::UNC-10::GFP) .

\section{$\underline{\text { CRISPR/Cas-9 endogenously tagged NRX-1::GFP }}$}

Strain PHX3578 nrx-1(syb3578) was generated in N2 animals by SunyBiotech. Linker sequence and GFP were inserted after exon 27 at the 3' end of C29A12.4a1/nrx-1. Sequence flanking the GFP is below.

5': CGGGAATGGAGTCGCAAAGAAAAAGGATTTTAAAGAGTGGTACGTAaaggtaccgcgggccc gggatccaccggtcgccaccatggtg

\section{3': TCCATTTCTTCAATCAAAACTCAATACAATGATGATTAAAAAATTCACTTTTGTCTGCAAA TTGCCACAACTTCAAAACGGGTAC}

PAM sequence is underlined

Synonymous mutations are bolded

Linker is in lower case letters

$n r x-1$ gene is CAPTIALIZED

\section{$\underline{\text { Hydrogel solution }}$}

Hydrogel was prepared as an aqueous solution of polyethylene glycol $(20 \% \mathrm{w} / \mathrm{v})$ and the photoinitiator Irgacure ( $0.5 \%$ stock, $0.1 \%$ working concentration), and stored at $4^{\circ} \mathrm{C}$ in the dark. 


\section{Confocal microscopy}

All strains were immobilized with sodium azide $(0.3 \mathrm{M})$ on a $2 \%$ or $5 \%$ agarose pad. Images were obtained using an Olympus BX51WI spinning disk confocal equipped with a $63 x$ objective. For time course analyses, newly hatched larvae were transferred to a seeded OP50 plate and maintained at $25^{\circ} \mathrm{C}$.

Long term live imaging of dendritic spines and F-actin. Nematodes were immobilized using $2 \mu \mathrm{L}$ $50 \mathrm{mM}$ muscimol in $10 \mu \mathrm{L} 20 \%$ PEG hydrogel solution. Once paralyzed, hydrogel was curated using a handheld UV Transilluminator (312 nm, 3 minutes). Z-stacks were acquired every 5 minutes for at least one hour.

Live imaging of synaptic vesicle and NRX-1 trafficking. Nematodes were immobilized using 50 $\mathrm{mM}$ muscimol on a $5 \%$ agarose pad. Cholinergic commissures were imaged using Perkin Elmer spinning disk confocal equipped with a $63 x$ objective using 100 ms exposure for 30 seconds.

\section{Confocal microscopy analysis}

All image analysis was conducted using ImageJ software (open source) within defined ROls using intensity threshold values determined from control experiments. ROls were located 15-30 $\mu \mathrm{m}$ anterior to DD1, DD2 or DD3 somas.

Spine analysis. Mature spines were quantified as protrusions from the dendrite $>0.3 \mu \mathrm{m}$ in length (measuring from the base to the tip of the protrusion). Spine density was defined as the number of spines/unit length within a selected ROI.

3D rendering was conducted using Imaris/bitplane 3D image analysis software. Morphological categories were determined based on criteria used in (Harris et al., 1992). The ImageJ line tool was used to measure the length and width of the spine 3D rendering. Based on these 
calculations, spines were placed into one of four morphological categories based on the following criteria: Stubby: middle width>total length; Thin: middle width=tip width; Mushroom: tip width>middle width; Branched: >1 spine head.

Fluorescence intensity analysis

F-actin/spine and F-actin/tubulin. The DD neurite was extracted using the line and line straighten functions. Total fluorescence intensity (utrophin/UtrCH, tubulin/TBA-1, ER/TRAM-1) on the dendritic shaft (line width 4) and below the dendritic shaft (line width 8) were measured. For soma fluorescence intensity, ROI was drawn around the perimeter of the soma. Synaptic marker and receptor cluster. Background fluorescence was subtracted, and the number and size of synaptic/receptor puncta were measured using the 'analyze particles' function. Confocal montages were assembled using the 'straighten to line' function in a $50 \mu \mathrm{m}$ region of the dorsal nerve cord.

\section{Live Imaging}

An event is defined as an incidence of spine destabilization/pruning, elongation/formation, or a stable spine during an entire live imaging recording. Z-stacks of DD1 dendrites were acquired every 5 minutes for 60-160 minutes. Numbers indicates number of events for each genotype at each timepoint.

\section{Calcium Imaging}

Animals were grown on plates seeded with OP50 containing 2.7 mM All-Trans Retinal (ATR).

Plates were stored at $4^{\circ} \mathrm{C}$ under dark conditions and used within one week. Imaging was carried out using L4 animals immobilized in hydrogel (Burnett et al., 2018). Animals were transferred to $7.5 \mu \mathrm{L}$ of the hydrogel mix placed on a silanized glass slide and covered with a glass slide.

Hydrogel was cured using a handheld UV Transilluminator (312 nm, 3 minutes). Post-curing, the 
covering slide was removed and replaced with a coverslip. Imaging was carried out using a Yokogawa CSU-X1-A1N spinning disk confocal system (Perkin Elmer) equipped with EM-CCD camera (Hamamatsu, C9100-50) and 63X oil immersion objective. Chrimson photoactivation $\left(\sim 30 \mathrm{~mW} / \mathrm{cm}^{2}\right)$ was achieved using a TTL-controlled $625 \mathrm{~nm}$ light guide coupled LED (Mightex Systems). A $556 \mathrm{~nm}$ BrightLine single-edge short-pass dichroic beam splitter was positioned in the light path (Semrock) (Figure S1.3). Data were acquired at $10 \mathrm{~Hz}$ for $15 \mathrm{~s}$ using Volocity software and binned at $1 \times 1$ during acquisition. Analysis was performed using ImageJ. The DD neurite process in each time series was extracted using the straighten function, background subtracted and photobleaching correction was carried out by fitting an exponential function to the data (CorrectBleach plugin). A smoothing function was applied to the data to enhance signal-to-noise. Individual spine ROls were identified using the mCherry fluorescence. Post imaging processing, pre-stimulus baseline fluorescence $\left(F_{0}\right)$ was calculated as the average of the data points in the first $4 \mathrm{~s}$ of the recording. Data was normalized to prestimulus baseline as $\Delta F / F_{0}$, where $\Delta F=F-F_{0}$. Peak $\Delta F / F_{0}$ was determined by fitting a Gaussian function to the $\Delta \mathrm{F} / \mathrm{F}_{0}$ time sequence using Multi peak 2.0 (Igor Pro, WaveMetrics). All collected data were analyzed, including failures (no response to stimulation). Control recordings were carried out in the absence of Retinal.

\section{Electron microscopy}

Staged L4 wild type (Pflp-13::ACR-12::GFP) hermaphrodites were subjected to high-pressure freeze fixation using a HPM10 high-pressure freezer. Hermaphrodites were slowly freeze substituted in $2 \%$ osmium tetroxide (OSO4), $0.1 \%$ uranyl acetate, $2 \% \mathrm{H} 2 \mathrm{O}$ in acetone fixative (Weimer, 2006) (Muller-Reichert et al., 2010). Samples were embedded in blocks, sectioned at $70 \mathrm{~nm}$-thick serial sections and subsequently collected on copper slot grids. Samples were poststained in $2.5 \%$ uranyl acetate in $70 \%$ methanol for 20 minutes and $1: 6$ lead citrate in $\mathrm{H}_{2} \mathrm{O}$ for 2 minutes at room temperature. Images were acquired using the Philips CM10 TEM. 
We identified and characterized 6 DD1 spines from a $35 \mu \mathrm{m}$ region of the ventral head region where the neurite emerges from the cell body of DD1 GABAergic motor neuron.

\section{Temperature shift experiments}

Wild type and unc-104(ce782)ts animals eggs were raised at $13.5^{\circ} \mathrm{C}$ until L3 or L4 stage as indicated, then shifted to $25^{\circ} \mathrm{C}$ for $16-20 \mathrm{hrs}$ prior to imaging. Animals were age-matched at the time of the temperature shift to account for developmental delays of unc-104(ce782)ts mutants. 


\section{References}

Aakalu, G., Smith, W. B., Nguyen, N., Jiang, C., \& Schuman, E. M. (2001). Dynamic visualization of local protein synthesis in hippocampal neurons. Neuron, 30(2), 489-502. doi:10.1016/s0896-6273(01)00295-1

Bohme, M. A., Beis, C., Reddy-Alla, S., Reynolds, E., Mampell, M. M., Grasskamp, A. T., . . Sigrist, S. J. (2016). Active zone scaffolds differentially accumulate Unc13 isoforms to tune $\mathrm{Ca}(2+)$ channel-vesicle coupling. Nat Neurosci, 19(10), 13111320. doi: $10.1038 / \mathrm{nn} .4364$

Borovac, J., Bosch, M., \& Okamoto, K. (2018). Regulation of actin dynamics during structural plasticity of dendritic spines: Signaling messengers and actin-binding proteins. Mol Cell Neurosci, 91, 122-130. doi:10.1016/j.mcn.2018.07.001

Burnett, K., Edsinger, E., \& Albrecht, D. R. (2018). Rapid and gentle hydrogel encapsulation of living organisms enables long-term microscopy over multiple hours. Commun Biol, 1, 73. doi:10.1038/s42003-018-0079-6

Chen, L. Y., Jiang, M., Zhang, B., Gokce, O., \& Sudhof, T. C. (2017). Conditional Deletion of All Neurexins Defines Diversity of Essential Synaptic Organizer Functions for Neurexins. Neuron, 94(3), 611-625 e614. doi:10.1016/j.neuron.2017.04.011

Chen, S. X., Tari, P. K., She, K., \& Haas, K. (2010). Neurexin-neuroligin cell adhesion complexes contribute to synaptotropic dendritogenesis via growth stabilization mechanisms in vivo. Neuron, 67(6), 967-983. doi:10.1016/j.neuron.2010.08.016

Chia, P. H., Chen, B., Li, P., Rosen, M. K., \& Shen, K. (2014). Local F-actin network links synapse formation and axon branching. Cell, 156(1-2), 208-220. doi:10.1016/j.cell.2013.12.009

Chia, P. H., Patel, M. R., \& Shen, K. (2012). NAB-1 instructs synapse assembly by linking adhesion molecules and F-actin to active zone proteins. Nat Neurosci, 15(2), 234242. doi: $10.1038 / \mathrm{nn} .2991$

Chirillo, M. A., Waters, M. S., Lindsey, L. F., Bourne, J. N., \& Harris, K. M. (2019). Local resources of polyribosomes and SER promote synapse enlargement and spine clustering after long-term potentiation in adult rat hippocampus. Sci Rep, 9(1), 3861. doi:10.1038/s41598-019-40520-x

Constance, W. D., Mukherjee, A., Fisher, Y. E., Pop, S., Blanc, E., Toyama, Y., \& Williams, D. W. (2018). Neurexin and Neuroligin-based adhesion complexes drive axonal arborisation growth independent of synaptic activity. eLife, 7. doi:10.7554/eLife.31659

Cuentas-Condori, A., Mulcahy, B., He, S., Palumbos, S., Zhen, M., \& Miller, D. M., III. (2019). C. elegans neurons have functional dendritic spines. eLife, 8, e47918. doi:10.7554/eLife.47918

Dai, Y., Taru, H., Deken, S. L., Grill, B., Ackley, B., Nonet, M. L., \& Jin, Y. (2006). SYD-2 Liprin-alpha organizes presynaptic active zone formation through ELKS. Nat Neurosci, 9(12), 1479-1487. doi:10.1038/nn1808

De Roo, M., Klauser, P., Garcia, P. M., Poglia, L., \& Muller, D. (2008). Spine dynamics and synapse remodeling during LTP and memory processes. Prog Brain Res, 169, 199-207. doi:10.1016/S0079-6123(07)00011-8

Edwards, S. L., Yorks, R. M., Morrison, L. M., Hoover, C. M., \& Miller, K. G. (2015). Synapse-Assembly Proteins Maintain Synaptic Vesicle Cluster Stability and 
Regulate Synaptic Vesicle Transport in Caenorhabditis elegans. Genetics, 201(1), 91-116. doi:10.1534/genetics.115.177337

Edwards, S. L., Yu, S. C., Hoover, C. M., Phillips, B. C., Richmond, J. E., \& Miller, K. G. (2013). An organelle gatekeeper function for Caenorhabditis elegans UNC-16 (JIP3) at the axon initial segment. Genetics, 194(1), 143-161. doi:10.1534/genetics.112.147348

Engert, F., \& Bonhoeffer, T. (1999). Dendritic spine changes associated with hippocampal long-term synaptic plasticity. Nature, 399(6731), 66-70. doi:10.1038/19978

Fouquet, W., Owald, D., Wichmann, C., Mertel, S., Depner, H., Dyba, M., . . Sigrist, S. J. (2009). Maturation of active zone assembly by Drosophila Bruchpilot. J Cell Biol, 186(1), 129-145. doi:10.1083/jcb.200812150

Friedman, H. V., Bresler, T., Garner, C. C., \& Ziv, N. E. (2000). Assembly of new individual excitatory synapses: time course and temporal order of synaptic molecule recruitment. Neuron, 27(1), 57-69. doi:10.1016/s0896-6273(00)00009-x

Gally, C., Eimer, S., Richmond, J. E., \& Bessereau, J. L. (2004). A transmembrane protein required for acetylcholine receptor clustering in Caenorhabditis elegans. Nature, 431(7008), 578-582. doi:10.1038/nature02893

Graf, E. R., Zhang, X., Jin, S. X., Linhoff, M. W., \& Craig, A. M. (2004). Neurexins induce differentiation of GABA and glutamate postsynaptic specializations via neuroligins. Cell, 119(7), 1013-1026. doi:10.1016/j.cell.2004.11.035

Gu, J., Firestein, B. L., \& Zheng, J. Q. (2008). Microtubules in dendritic spine development. J Neurosci, 28(46), 12120-12124. doi:10.1523/JNEUROSCI.2509-08.2008

Haklai-Topper, L., Soutschek, J., Sabanay, H., Scheel, J., Hobert, O., \& Peles, E. (2011). The neurexin superfamily of Caenorhabditis elegans. Gene Expr Patterns, 11(12), 144-150. doi:10.1016/j.gep.2010.10.008

Hall, D. H., \& Hedgecock, E. M. (1991). Kinesin-related gene unc-104 is required for axonal transport of synaptic vesicles in C. elegans. Cell, 65(5), 837-847. doi:10.1016/0092-8674(91)90391-b

Hall, D. H., \& Russell, R. L. (1991). The posterior nervous system of the nematode Caenorhabditis elegans: serial reconstruction of identified neurons and complete pattern of synaptic interactions. J Neurosci, 11(1), 1-22. Retrieved from https://www.ncbi.nlm.nih.gov/pubmed/1986064

Harris, K. M., Jensen, F. E., \& Tsao, B. (1992). Three-dimensional structure of dendritic spines and synapses in rat hippocampus (CA1) at postnatal day 15 and adult ages: implications for the maturation of synaptic physiology and long-term potentiation. J Neurosci, 12(7), 2685-2705. Retrieved from https://www.ncbi.nlm.nih.gov/pubmed/1613552

He, S., Cuentas-Condori, A., \& Miller, D. M., 3rd. (2019). NATF (Native and TissueSpecific Fluorescence): A Strategy for Bright, Tissue-Specific GFP Labeling of Native Proteins in Caenorhabditis elegans. Genetics, 212(2), 387-395. doi:10.1534/genetics.119.302063

Hendi, A., Kurashina, M., \& Mizumoto, K. (2019). Intrinsic and extrinsic mechanisms of synapse formation and specificity in C. elegans. Cell Mol Life Sci, 76(14), 27192738. doi:10.1007/s00018-019-03109-1 
Hendricks, M., Ha, H., Maffey, N., \& Zhang, Y. (2012). Compartmentalized calcium dynamics in a C. elegans interneuron encode head movement. Nature, 487(7405), 99-103. doi:10.1038/nature11081

Hu, X., Viesselmann, C., Nam, S., Merriam, E., \& Dent, E. W. (2008). Activity-dependent dynamic microtubule invasion of dendritic spines. J Neurosci, 28(49), 1309413105. doi:10.1523/JNEUROSCI.3074-08.2008

Jaworski, J., Kapitein, L. C., Gouveia, S. M., Dortland, B. R., Wulf, P. S., Grigoriev, I., ... . Hoogenraad, C. C. (2009). Dynamic microtubules regulate dendritic spine morphology and synaptic plasticity. Neuron, 61(1), 85-100. doi:10.1016/j.neuron.2008.11.013

Karagas, N. E., \& Venkatachalam, K. (2019). Roles for the Endoplasmic Reticulum in Regulation of Neuronal Calcium Homeostasis. Cells, 8(10). doi:10.3390/cells8101232

Kasem, E., Kurihara, T., \& Tabuchi, K. (2018). Neurexins and neuropsychiatric disorders. Neurosci Res, 127, 53-60. doi:10.1016/j.neures.2017.10.012

Koushika, S. P., Richmond, J. E., Hadwiger, G., Weimer, R. M., Jorgensen, E. M., \& Nonet, M. L. (2001). A post-docking role for active zone protein Rim. Nat Neurosci, 4(10), 997-1005. doi:10.1038/nn732

Kumar, J., Choudhary, B. C., Metpally, R., Zheng, Q., Nonet, M. L., Ramanathan, S., .. . Koushika, S. P. (2010). The Caenorhabditis elegans Kinesin-3 motor UNC104/KIF1A is degraded upon loss of specific binding to cargo. PLoS Genet, 6(11), e1001200. doi:10.1371/journal.pgen.1001200

Kurshan, P. T., Merrill, S. A., Dong, Y., Ding, C., Hammarlund, M., Bai, J., . . . Shen, K. (2018). gamma-Neurexin and Frizzled Mediate Parallel Synapse Assembly Pathways Antagonized by Receptor Endocytosis. Neuron, 100(1), 150-166 e154. doi:10.1016/j.neuron.2018.09.007

Kurup, N., \& Jin, Y. (2016). Neural circuit rewiring: insights from DD synapse remodeling. Worm, 5(1), e1129486. doi:10.1080/21624054.2015.1129486

Li, J., Ashley, J., Budnik, V., \& Bhat, M. A. (2007). Crucial role of Drosophila neurexin in proper active zone apposition to postsynaptic densities, synaptic growth, and synaptic transmission. Neuron, 55(5), 741-755. doi:10.1016/j.neuron.2007.08.002

Li, Z., Okamoto, K., Hayashi, Y., \& Sheng, M. (2004). The importance of dendritic mitochondria in the morphogenesis and plasticity of spines and synapses. Cell, 119(6), 873-887. doi:10.1016/j.cell.2004.11.003

Lipton, D. M., Maeder, C. I., \& Shen, K. (2018). Rapid Assembly of Presynaptic Materials behind the Growth Cone in Dopaminergic Neurons Is Mediated by Precise Regulation of Axonal Transport. Cell Rep, 24(10), 2709-2722. doi:10.1016/j.celrep.2018.07.096

Liu, Q. A., \& Shio, H. (2008). Mitochondrial morphogenesis, dendrite development, and synapse formation in cerebellum require both Bcl-w and the glutamate receptor delta2. PLoS Genet, 4(6), e1000097. doi:10.1371/journal.pgen.1000097

Lu, W., Bushong, E. A., Shih, T. P., Ellisman, M. H., \& Nicoll, R. A. (2013). The cellautonomous role of excitatory synaptic transmission in the regulation of neuronal structure and function. Neuron, 78(3), 433-439. doi:10.1016/j.neuron.2013.02.030 
Luo, F., Sclip, A., Jiang, M., \& Sudhof, T. C. (2020). Neurexins cluster $\mathrm{Ca}(2+)$ channels within the presynaptic active zone. EMBO J, 39(7), e103208. doi:10.15252/embj.2019103208

McDonald, N. A., Fetter, R. D., \& Shen, K. (2020). Assembly of synaptic active zones requires phase separation of scaffold molecules. Nature, 588(7838), 454-458. doi:10.1038/s41586-020-2942-0

Mello, C. C., Kramer, J. M., Stinchcomb, D., \& Ambros, V. (1991). Efficient gene transfer in C.elegans: extrachromosomal maintenance and integration of transforming sequences. EMBO J, 10(12), 3959-3970. Retrieved from https://www.ncbi.nlm.nih.gov/pubmed/1935914

Meng, L., Mulcahy, B., Cook, S. J., Neubauer, M., Wan, A., Jin, Y., \& Yan, D. (2015). The Cell Death Pathway Regulates Synapse Elimination through Cleavage of Gelsolin in Caenorhabditis elegans Neurons. Cell Rep, 11(11), 1737-1748. doi:10.1016/j.celrep.2015.05.031

Missler, M., \& Sudhof, T. C. (1998). Neurexins: three genes and 1001 products. Trends Genet, 14(1), 20-26. doi:10.1016/S0168-9525(97)01324-3

Muller-Reichert, T., Mancuso, J., Lich, B., \& McDonald, K. (2010). Three-dimensional reconstruction methods for Caenorhabditis elegans ultrastructure. Methods Cell Biol, 96, 331-361. doi:10.1016/S0091-679X(10)96015-9

Nagerl, U. V., Kostinger, G., Anderson, J. C., Martin, K. A., \& Bonhoeffer, T. (2007). Protracted synaptogenesis after activity-dependent spinogenesis in hippocampal neurons. J Neurosci, 27(30), 8149-8156. doi:10.1523/JNEUROSCI.0511-07.2007

Neupert, C., Schneider, R., Klatt, O., Reissner, C., Repetto, D., Biermann, B., . . Heine, M. (2015). Regulated Dynamic Trafficking of Neurexins Inside and Outside of Synaptic Terminals. J Neurosci, 35(40), 13629-13647. doi:10.1523/JNEUROSCI.4041-14.2015

Oliver, D., Alexander, K., \& Francis, M. M. (2018). Molecular Mechanisms Directing Spine Outgrowth and Synaptic Partner Selection in Caenorhabditis elegans. J Exp Neurosci, 12, 1179069518816088. doi:10.1177/1179069518816088

Owald, D., Khorramshahi, O., Gupta, V. K., Banovic, D., Depner, H., Fouquet, W., . . . Sigrist, S. J. (2012). Cooperation of Syd-1 with Neurexin synchronizes pre- with postsynaptic assembly. Nat Neurosci, 15(9), 1219-1226. doi:10.1038/nn.3183

Patel, M. R., Lehrman, E. K., Poon, V. Y., Crump, J. G., Zhen, M., Bargmann, C. I., \& Shen, K. (2006). Hierarchical assembly of presynaptic components in defined C. elegans synapses. Nat Neurosci, 9(12), 1488-1498. doi:10.1038/nn1806

Perez-Alvarez, A., Yin, S., Schulze, C., Hammer, J. A., Wagner, W., \& Oertner, T. G. (2020). Endoplasmic reticulum visits highly active spines and prevents runaway potentiation of synapses. Nat Commun, 11(1), 5083. doi:10.1038/s41467-02018889-5

Philbrook, A., Ramachandran, S., Lambert, C. M., Oliver, D., Florman, J., Alkema, M. J., . . . Francis, M. M. (2018). Neurexin directs partner-specific synaptic connectivity in C. elegans. eLife, 7. doi:10.7554/eLife.35692

Richmond, J. E., Davis, W. S., \& Jorgensen, E. M. (1999). UNC-13 is required for synaptic vesicle fusion in C. elegans. Nat Neurosci, 2(11), 959-964. doi:10.1038/14755 
Rolls, M. M., Hall, D. H., Victor, M., Stelzer, E. H., \& Rapoport, T. A. (2002). Targeting of rough endoplasmic reticulum membrane proteins and ribosomes in invertebrate neurons. Mol Biol Cell, 13(5), 1778-1791. doi:10.1091/mbc.01-10-0514

Sala, C., \& Segal, M. (2014). Dendritic spines: the locus of structural and functional plasticity. Physiol Rev, 94(1), 141-188. doi:10.1152/physrev.00012.2013

Sando, R., Bushong, E., Zhu, Y., Huang, M., Considine, C., Phan, S., . . Maximov, A. (2017). Assembly of Excitatory Synapses in the Absence of Glutamatergic Neurotransmission. Neuron, 94(2), 312-321 e313. doi:10.1016/j.neuron.2017.03.047

Saneyoshi, T., Fortin, D. A., \& Soderling, T. R. (2010). Regulation of spine and synapse formation by activity-dependent intracellular signaling pathways. Curr Opin Neurobiol, 20(1), 108-115. doi:10.1016/j.conb.2009.09.013

Sarasija, S., \& Norman, K. R. (2015). A gamma-Secretase Independent Role for Presenilin in Calcium Homeostasis Impacts Mitochondrial Function and Morphology in Caenorhabditis elegans. Genetics, 201(4), 1453-1466. doi:10.1534/genetics.115.182808

Sigler, A., Oh, W. C., Imig, C., Altas, B., Kawabe, H., Cooper, B. H., . . Brose, N. (2017). Formation and Maintenance of Functional Spines in the Absence of Presynaptic Glutamate Release. Neuron, 94(2), 304-311 e304. doi:10.1016/j.neuron.2017.03.029

Steward, O., \& Levy, W. B. (1982). Preferential localization of polyribosomes under the base of dendritic spines in granule cells of the dentate gyrus. J Neurosci, 2(3), 284291. Retrieved from https://www.ncbi.nlm.nih.gov/pubmed/7062109

Sudhof, T. C. (2008). Neuroligins and neurexins link synaptic function to cognitive disease. Nature, 455(7215), 903-911. doi:10.1038/nature07456

Sudhof, T. C. (2017). Synaptic Neurexin Complexes: A Molecular Code for the Logic of Neural Circuits. Cell, 171(4), 745-769. doi:10.1016/j.cell.2017.10.024

Tonnesen, J., \& Nagerl, U. V. (2016). Dendritic Spines as Tunable Regulators of Synaptic Signals. Front Psychiatry, 7, 101. doi:10.3389/fpsyt.2016.00101

Vos, M., Lauwers, E., \& Verstreken, P. (2010). Synaptic mitochondria in synaptic transmission and organization of vesicle pools in health and disease. Front Synaptic Neurosci, 2, 139. doi:10.3389/fnsyn.2010.00139

Weimer, R. M. (2006). Preservation of C. elegans tissue via high-pressure freezing and freeze-substitution for ultrastructural analysis and immunocytochemistry. Methods Mol Biol, 351, 203-221. doi:10.1385/1-59745-151-7:203

White, J. (2018). Clues to basis of exploratory behaviour of the C. elegans snout from head somatotropy. Philos Trans $R$ Soc Lond B Biol Sci, 373(1758). doi:10.1098/rstb.2017.0367

White, J. G., Albertson, D. G., \& Anness, M. A. (1978). Connectivity changes in a class of motoneurone during the development of a nematode. Nature, 271(5647), 764766. doi:10.1038/271764a0

White, J. G., Southgate, E., Thomson, J. N., \& Brenner, S. (1976). The structure of the ventral nerve cord of Caenorhabditis elegans. Philos Trans R Soc Lond B Biol Sci, 275(938), 327-348. doi:10.1098/rstb.1976.0086 
White, J. G., Southgate, E., Thomson, J. N., \& Brenner, S. (1986). The structure of the nervous system of the nematode Caenorhabditis elegans. Philos Trans $R$ Soc Lond B Biol Sci, 314(1165), 1-340. doi:10.1098/rstb.1986.0056

Witvliet, D., Mulcahy, B., Mitchell, J. K., Meirovitch, Y., Berger, D. R., Wu, Y., . . Zhen, M. (2021). Connectomes across development reveal principles of brain maturation. bioRxiv, 2020.2004.2030.066209. doi:10.1101/2020.04.30.066209

Xuan, Z., Manning, L., Nelson, J., Richmond, J. E., Colon-Ramos, D. A., Shen, K., \& Kurshan, P. T. (2017). Clarinet (CLA-1), a novel active zone protein required for synaptic vesicle clustering and release. eLife, 6. doi:10.7554/eLife.29276

Yan, J., Chao, D. L., Toba, S., Koyasako, K., Yasunaga, T., Hirotsune, S., \& Shen, K. (2013). Kinesin-1 regulates dendrite microtubule polarity in Caenorhabditis elegans. eLife, 2, e00133. doi:10.7554/eLife.00133

Zahn, T. R., Angleson, J. K., MacMorris, M. A., Domke, E., Hutton, J. F., Schwartz, C., \& Hutton, J. C. (2004). Dense core vesicle dynamics in Caenorhabditis elegans neurons and the role of kinesin UNC-104. Traffic, 5(7), 544-559. doi:10.1111/j.1600-0854.2004.00195.x

Zhen, M., \& Jin, Y. (1999). The liprin protein SYD-2 regulates the differentiation of presynaptic termini in C. elegans. Nature, 401(6751), 371-375. doi:10.1038/43886

Zito, K., Scheuss, V., Knott, G., Hill, T., \& Svoboda, K. (2009). Rapid functional maturation of nascent dendritic spines. Neuron, 61(2), 247-258. doi:10.1016/j.neuron.2008.10.054 

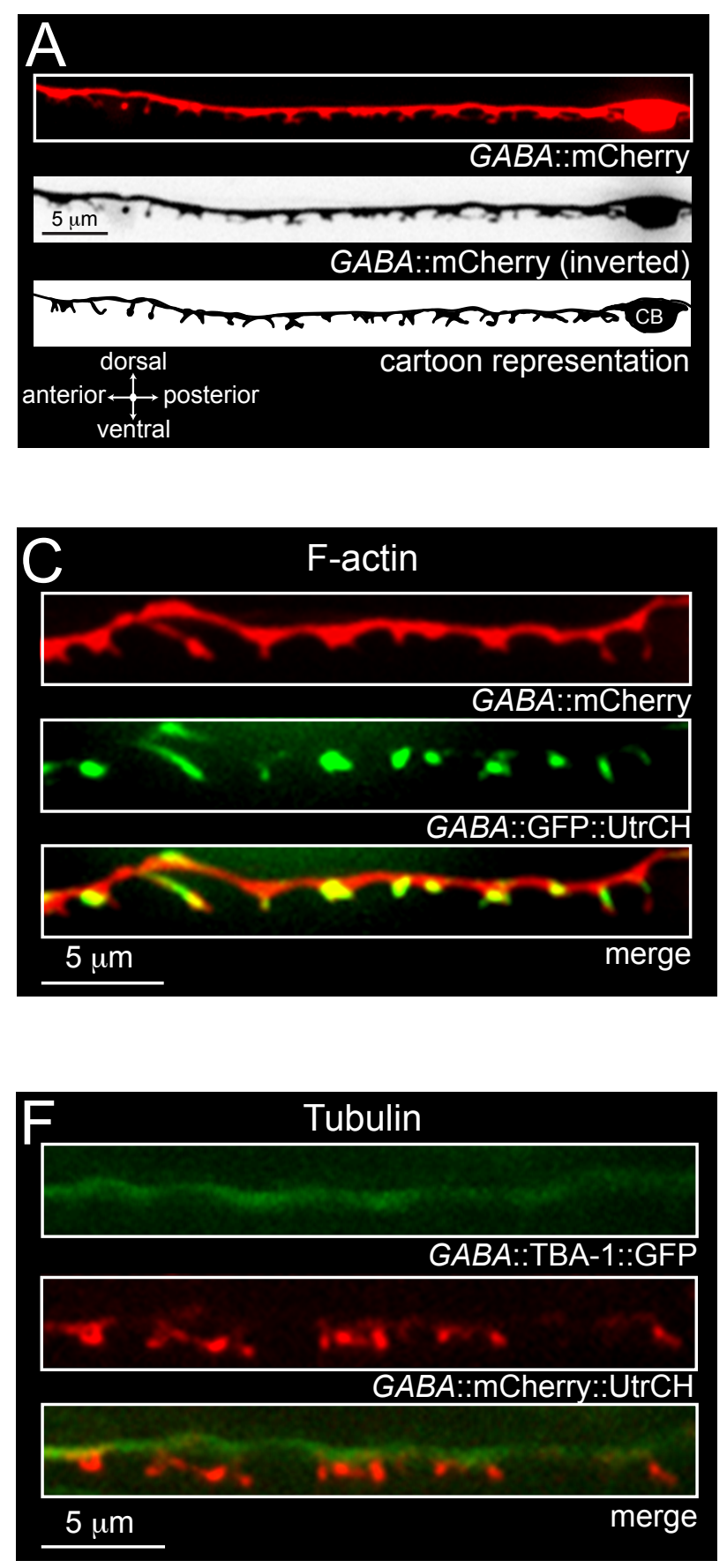

D

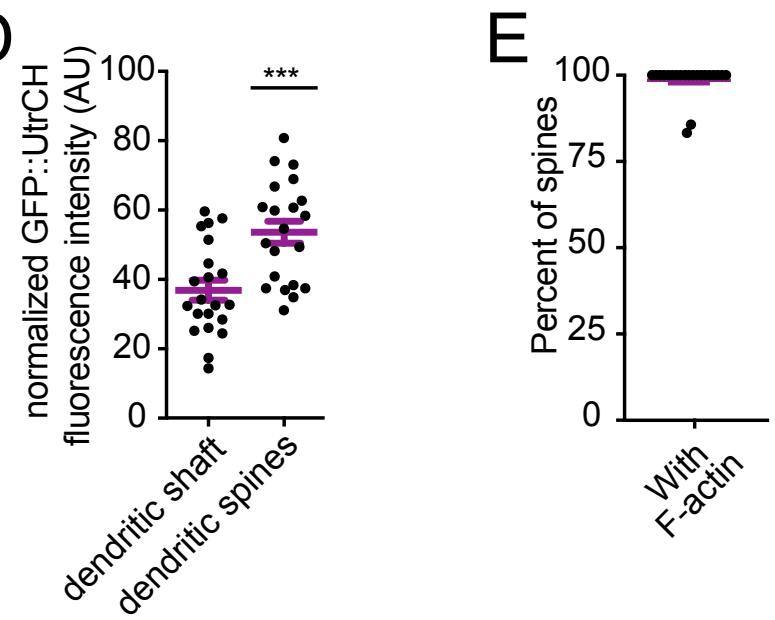

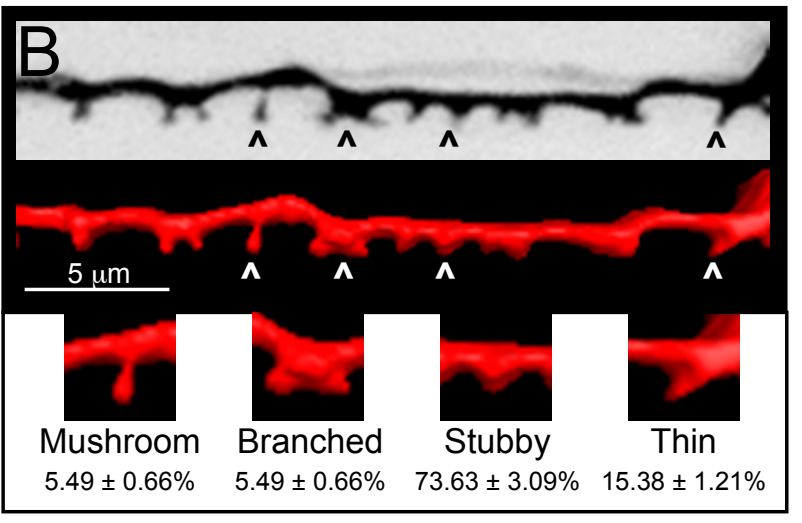
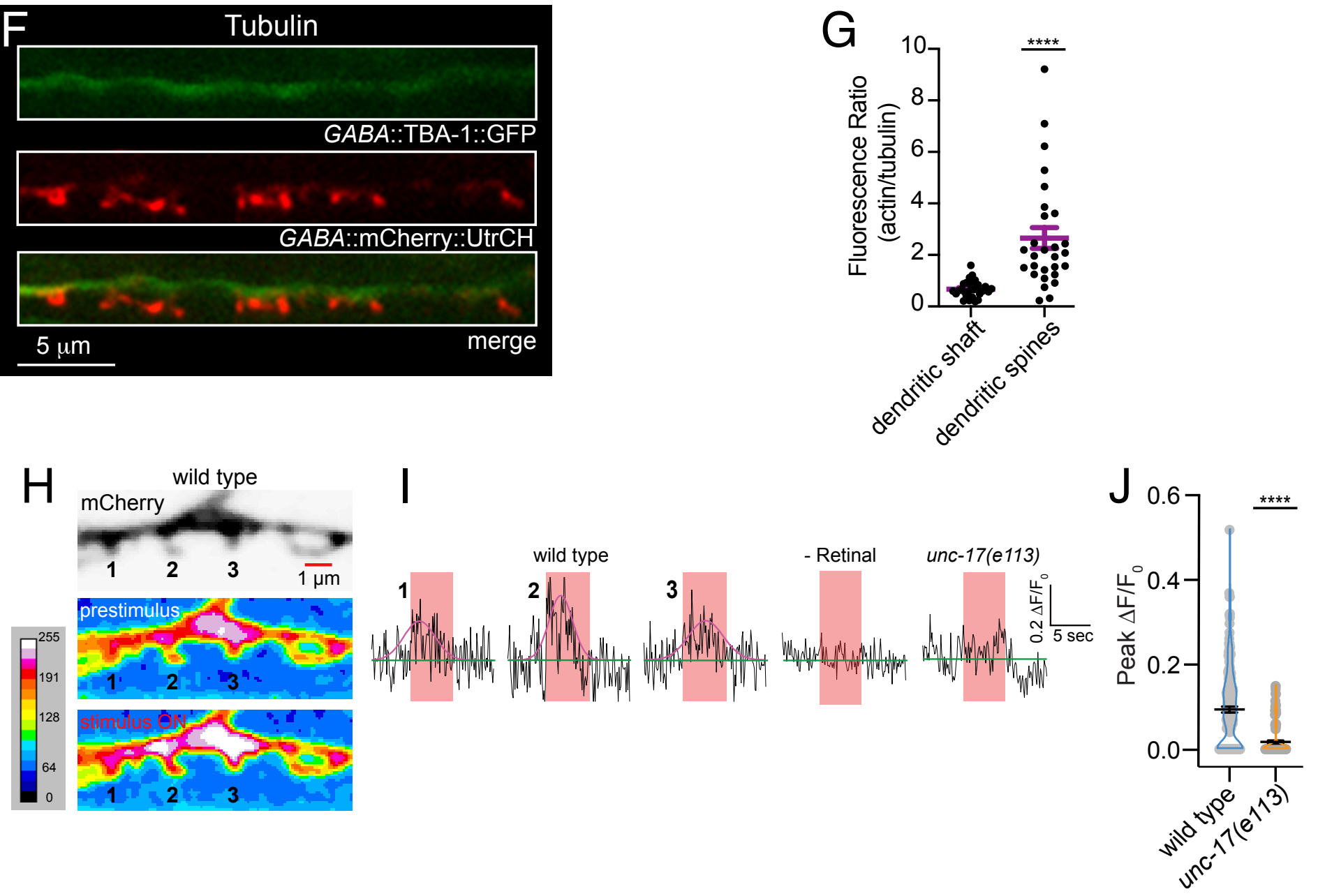
Figure 1. C. elegans GABAergic DD neuron dendritic spines share characteristics with dendritic spines in vertebrates.

(A) Representative fluorescent images and cartoon representation of the ventral nerve cord/dendrite of a single L4 stage GABAergic DD (DD2) motor neuron with ventrally projecting dendritic spines. CB, cell body.

(B) Fluorescent image (top, inverted LUT) and 3D rendering (middle and insets) of DD dendritic spines from an animal expressing Pflp-13::mCherry, shows diverse spine morphologies. DD dendritic spines share similar morphologies with mammalian dendritic spines: mushroom (5.49 $\pm 0.66 \%)$, branched $(5.49 \pm 0.66 \%)$, stubby $(73.63 \pm 3.09 \%)$, and thin $(15.38 \pm 1.21 \%)$. Arrowheads, spine insets. $n=83$ dendritic spines from 11 animals, measurements, percentage $\pm \mathrm{SD}$.

(C) Representative images of DD dendritic spines from animal co-expressing Pflp13::mCherry (labeling dendrite, red) and PfIp-13::GFP::UtrCH (labeling F-actin, green). Factin is highly enriched in DD dendritic spines.

(D) Scatterplot of F-actin/GFP::UtrCH fluorescence in dendritic shaft compared to dendritic spines, normalized to total fluorescence within the ROI. Bars indicate mean \pm SEM. Student's t-test, ${ }^{* *} p<0.001, \mathrm{n}=21$ animals.

(E) Scatterplot of the percentage of spines with F-actin. Bars, mean \pm SEM, $n=21$ animals.

(F) Fluorescent images of tubulin (Pflp-13::TBA-1::GFP) (green) and actin (Pflp13::mCherry::UtrCH) (red) in DD neurons. Dendritic spines are highly enriched with Factin while tubulin occupies the main dendritic process.

(G) Quantification of fluorescence ratio of actin/tubulin in the dendritic shaft versus dendritic spines. Student's t-test, ${ }^{* * *} p<0.0001, \mathrm{n}=31$ spines from 6 animals.

(H) Calcium transients recorded from wild type GABAergic DD motor neuron dendrites expressing myrGCaMP6f (Pflp-13::myrGCaMP6f::SL2::mCherry) during stimulation of presynaptic cholinergic motor neurons (Pacr-2::Chrimson). Top, representative image 
showing dendritic spines identified by mCherry fluorescence (inverted LUT). Representative maximum intensity projection heat maps showing calcium fluxes immediately prior to stimulation (prestimulus, middle panel) and during stimulus (ON, bottom panel). Heat maps were generated by maximum intensity projection of myrGCaMP6f fluorescence from the first 4 seconds of recording (prestimulus) and the $5 \mathrm{~s}$ period of stimulation (red sharded bar in I). Numbering corresponds to representative traces in Figure 11.

(I) Representative evoked responses for indicated dendritic spines in wild type animals (wild type 1, 2 and 3, Figure 1H), animals grown in the absence of retinal (-Retinal), and unc17(e113) mutants. Calcium responses were continuously recorded at $10 \mathrm{~Hz}$ for $15 \mathrm{~s}$. Prestimulus calcium fluxes were recorded for $5 \mathrm{~s}$, followed by stimulation $(625 \mathrm{~nm}, \sim 30$ $\mathrm{mW} / \mathrm{cm}^{2}$ ) of cholinergic motor neurons for $5 \mathrm{~s}$. All data was normalized to prestimulus $\left(\Delta F / F_{0}\right)$. Red shaded bar indicates duration of stimulation. Pink traces indicate Gaussian fits to responses $\left(\Delta F / F_{0}\right)$ during stimulation.

(J) Scatter plot showing peak $\Delta \mathrm{F} / \mathrm{F}_{0}$ of responses during stimulation in wild type or unc17(e113) mutants. Bars, mean \pm SEM. Statistical analysis, Student's t-test, ${ }^{* * * *} p<0.0001 . \mathrm{n} \geq 10$ animals. 

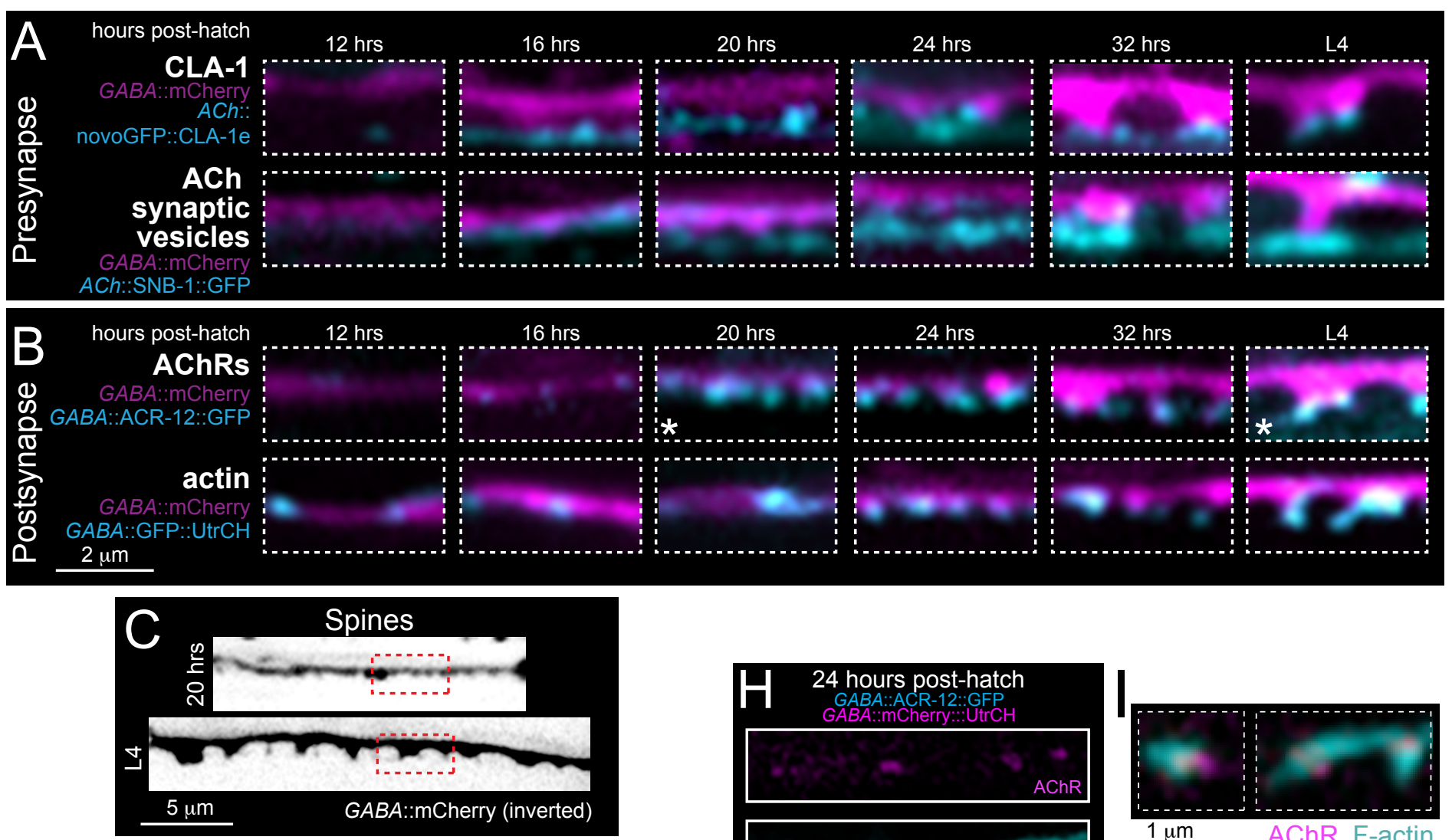

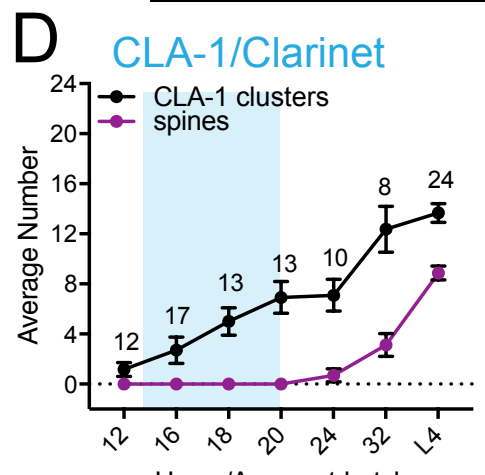

F Hours/Age post-hat

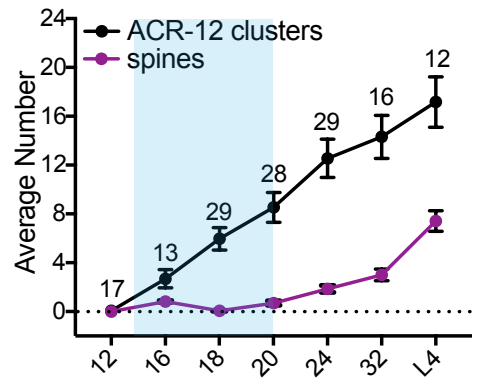

Hours/Age post-hatch

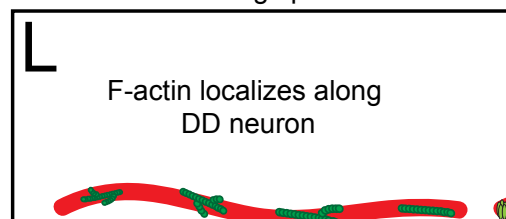

E

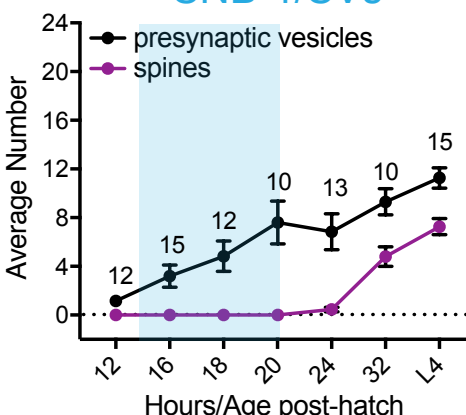

$G$

$\left.{ }^{24}\right] \rightarrow-F-a c t i n$ clusters

每

Hours/Age post-hatch

AChRs traffic along the dendritic shaft. Presynaptic vesicles/proteins form along axon of newly born ACh neurons
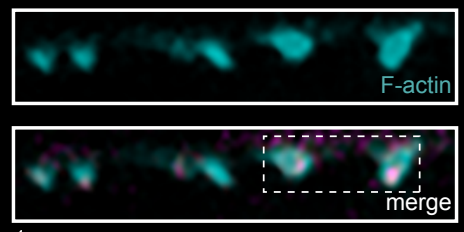

$1 \mu \mathrm{m}$
AChRs and F-actin migrate into immature spiny protrusions and presynaptic proteins begin to cluster
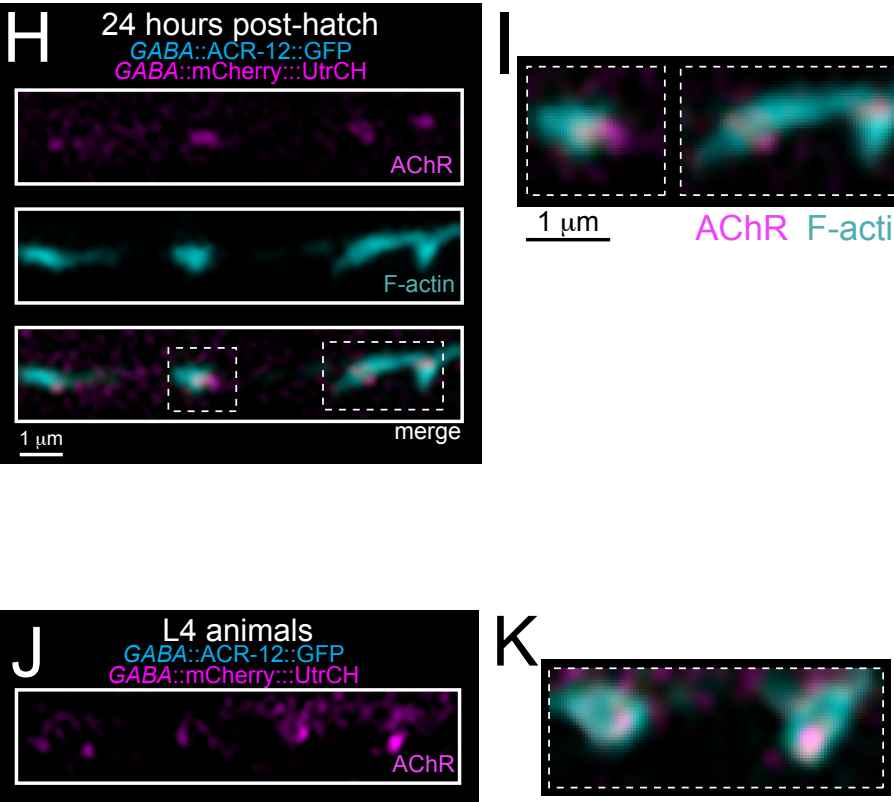

$1 \mu \mathrm{m}$ AChR F-actin
$1 \mu \mathrm{m}$ AChR F-actin

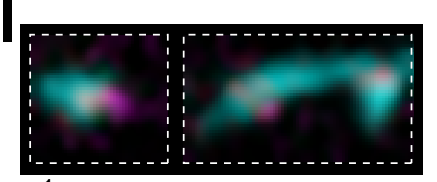

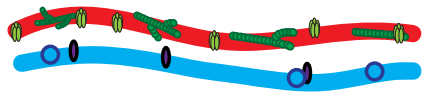

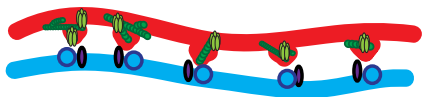

development

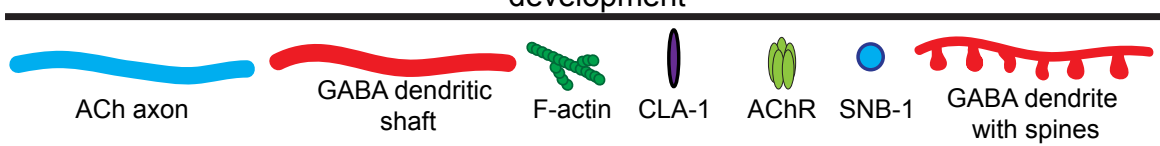

F-actin enriched dendritic spines elongate near sites of mature active zones

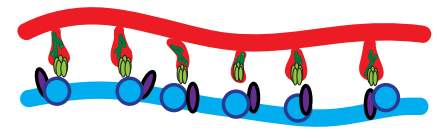


Figure 2. Temporal order of molecular events during synapse and spine development.

(A) Fluorescent images of GABAergic dendrites at 12, 16, 20, 24, and 32 hours after hatch and at L4 stage ( $42-50$ hours post-hatch). Animals express a GABAergic dendrite marker (Pflp-13::mCherry) with either presynaptic active zone (Punc-17 $\beta:$ :GFPnovo2::CLA-1e) (top) or synaptic vesicle marker (Pacr-5::SNB-1::GFP) (bottom). Images are pseudo colored to indicate spines (magenta) and presynaptic CLA-1 or vesicle clusters (cyan).

(B) Fluorescent images of GABAergic dendrites at 12, 16, 20, 24, and 32 hours after hatch and L4 animals ( 42-50 hours post-hatch). Animals express a GABAergic dendrite marker (Pflp-13::mCherry) with either postsynaptic AChR (Punc-47::ACR-12::GFP, top) or postsynaptic actin (Pflp-13::GFP::UtrCH, bottom) markers. Images are pseudo colored to indicate spines (magenta) and postsynaptic AChR or F-actin (cyan). White asterisks (*) indicate images used in Figure 2C.

(C) Fluorescent images (inverted LUT) of GABAergic dendrites at 20 hours after hatch and L4 stage ( 42-50 hours post-hatch) as indicated. Animals express a GABAergic dendrite marker (Pflp-13::mCherry). Dashed red boxes indicate regions used in Figure 2B (asterisks).

(D-G) Quantification of spine number (purple) relative to clusters of active zone protein/CLA-1 (D), synaptic vesicle/SNB-1 (E), AChR/ACR-12 (F), and F-actin/UtrCH (G) (black) through development. Clusters of each of these markers are visible well prior to spine outgrowth. Blue shading indicates the approximate period of DD remodeling. For this and subsequent figures, numbering indicates animals quantified at each timepoint for each genotype. Data points and bars indicate mean \pm SEM.

(H) Fluorescent confocal images of DD1 AChR/ACR-12 (Pflp-13::ACR-12::GFP, magenta) and F-actin clusters (Pflp-13::mCherry::UtrCH, cyan) 24 hours after hatch. Dotted rectangle indicates high magnification image shown in $2 \mathrm{l}$.

(I) Expanded view of DD1 dendrites 24 hours after hatch from Figure $2 \mathrm{H}$. 
(J) Fluorescent confocal images of DD1 AChR/ACR-12 (Pflp-13::ACR-12::GFP, magenta) and F-actin clusters (Pflp-13::mCherry::UtrCH, cyan) at L4 stage. Dotted rectangle indicates high magnification image shown in $2 \mathrm{~K}$.

(K) Expanded view of DD1 dendrites 24 hours after hatch from Figure 2J.

(L) Cartoon summary of the order of events during development of synapses onto DD neurons. In early development, presynaptic vesicles (SNB-1) and active zone protein (CLA-1) localize along presynaptic terminals while postsynaptic F-actin and postsynaptic receptors cluster along the postsynaptic process. Following their formation, dendritic spines begin to extend. Spines continue to elongate throughout development and into the mature circuit (L4). 
Table 1. Analysis of dendritic spines and cholinergic receptors in mutant strains with altered synaptic activity.

Dendritic spine number, spine length, ACR-12/AChR cluster number, and AChR cluster size at L4 stage normalized to the same measurements from wild type are shown (mean \pm SEM) for each genotype (ACR-2/nAChR UNC-2/CaV2 $\alpha$, UNC-13/MUNC-13, UNC-17/VAChT, UNC-18/MUNC18, UNC-31/CAPS). Mutations that affected synaptic activity had little effect on the density of spines or AChR clusters, but had variable effects on spine length and AChR cluster size. Oneway AVOVA, Dunnett's multiple comparisons test, ${ }^{*} p<0.05,{ }^{* *} p<0.01,{ }^{* * *} p<0.001,{ }^{* * *} p<0.0001$.

\begin{tabular}{|c|c|c|c|c|c|c|c|c|c|c|c|c|}
\hline \multirow[b]{2}{*}{ Genotype } & \multicolumn{6}{|c|}{ Spines } & \multicolumn{6}{|c|}{ AChR/receptors } \\
\hline & $\begin{array}{c}\text { Normalized } \\
\text { spine } \\
\text { number } \\
\pm \text { SEM (\%) } \\
\end{array}$ & $\mathrm{N}$ & $\begin{array}{c}\mathrm{P} \\
\text { value }\end{array}$ & $\begin{array}{c}\text { Normalized } \\
\text { spine } \\
\text { length } \\
\pm \text { SEM (\%) } \\
\end{array}$ & $\mathrm{N}$ & $\begin{array}{c}\mathrm{P} \\
\text { value }\end{array}$ & \begin{tabular}{|c|} 
Normalized \\
ACR-12 \\
cluster number \\
\pm SEM $(\%)$ \\
\end{tabular} & $\mathrm{N}$ & $\begin{array}{c}P \\
\text { value }\end{array}$ & $\begin{array}{l}\text { Normalized } \\
\text { ACR-12 } \\
\text { cluster size } \\
\pm \text { SEM }(\%) \\
\end{array}$ & $N$ & $\begin{array}{c}\mathrm{P} \\
\text { value }\end{array}$ \\
\hline wild type & $100.0 \pm 4.1$ & 58 & ns & $100.0 \pm 2.1$ & 404 & ns & $100.0 \pm 6.3$ & 17 & ns & $100.0 \pm 5.1$ & 220 & ns \\
\hline acr-2(ok1887) & $86.2 \pm 9.1$ & 24 & ns & $77.1 \pm 2.4$ & 141 & $* * * *$ & $94.6 \pm 10.4$ & 20 & ns & $109.8 \pm 5.9$ & 245 & ns \\
\hline acr-2(n2420)gf & $52.9 \pm 5.9$ & 26 & $* * * *$ & $83.8 \pm 2.6$ & 135 & ** & $75.6 \pm 6.3$ & 18 & ns & $81.3 \pm 5.4$ & 178 & ns \\
\hline unc-2(e55) & $81.8 \pm 7.6$ & 29 & ns & $90.9 \pm 3.7$ & 122 & ns & $77.2 \pm 9.5$ & 18 & ns & $83.9 \pm 5.7$ & 172 & ns \\
\hline$u n c-2(z f 35) g f$ & $81.7 \pm 7.2$ & 22 & ns & $114.7 \pm 4.8$ & 117 & * & $120.0 \pm 7.2$ & 20 & ns & $130.1 \pm 7.2$ & 312 & ** \\
\hline unc-13(e51) & $62.1 \pm 8.1$ & 18 & ** & $121.5 \pm 7.4$ & 78 & *** & $85.0 \pm 10.6$ & 19 & ns & $74.8 \pm 4.4$ & 210 & ns \\
\hline unc-17(e113) & $104.5 \pm 6.8$ & 12 & ns & $94.8 \pm 4.4$ & 83 & ns & $101.6 \pm 7.1$ & 24 & ns & $119.8 \pm 5.7$ & 317 & ns \\
\hline unc-18(e234) & $76.9 \pm 10.0$ & 11 & ns & $98.5 \pm 3.9$ & 59 & ns & $96.4 \pm 9.9$ & 19 & ns & $113.3 \pm 6.5$ & 238 & ns \\
\hline unc-31(e169) & $97.6 \pm 5.7$ & 22 & ns & $106.3 \pm 3.6$ & 166 & ns & $107.0 \pm 8.8$ & 21 & ns & $123.9 \pm 5.9$ & 291 & * \\
\hline
\end{tabular}



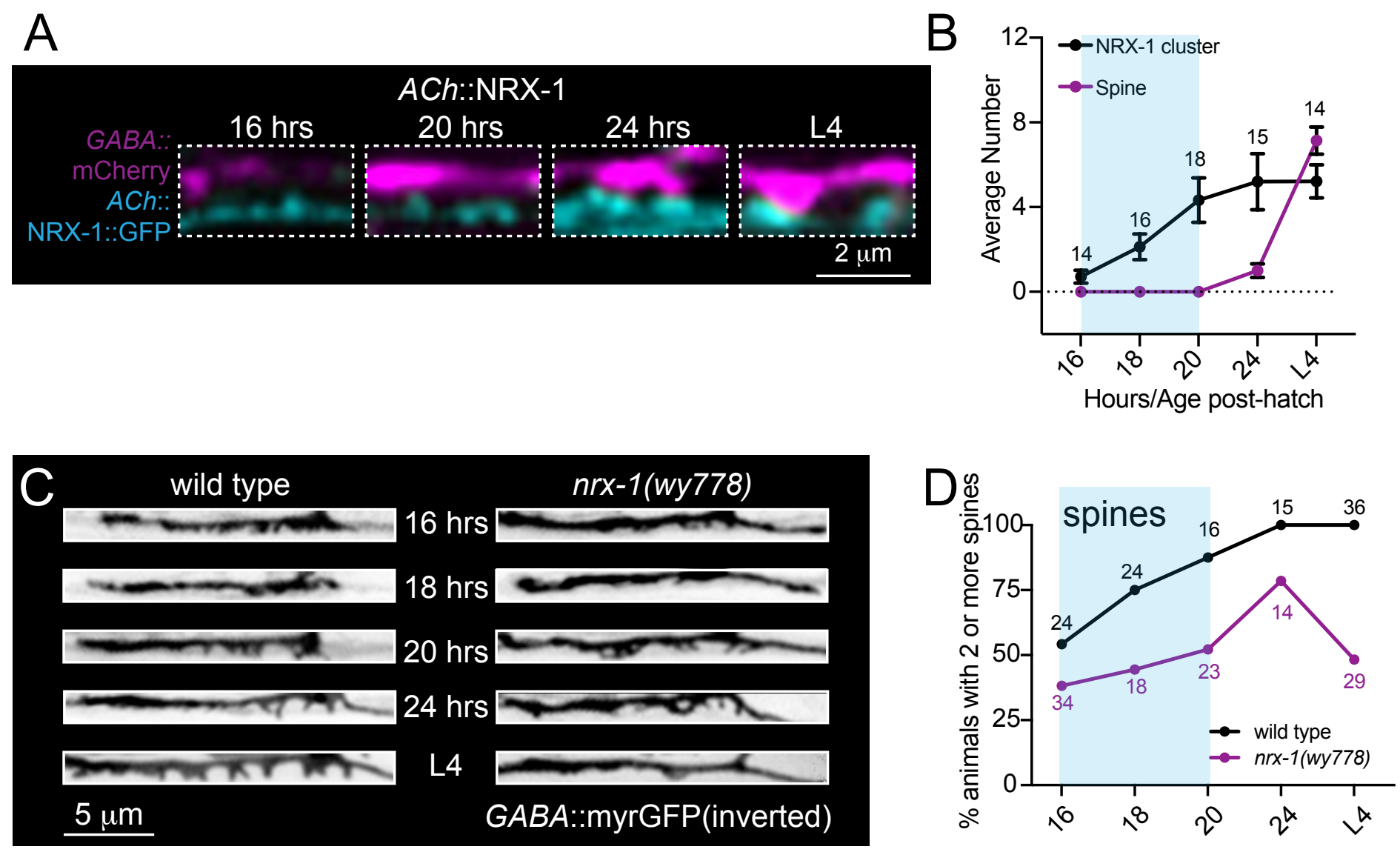

Hours/Age post-hatch
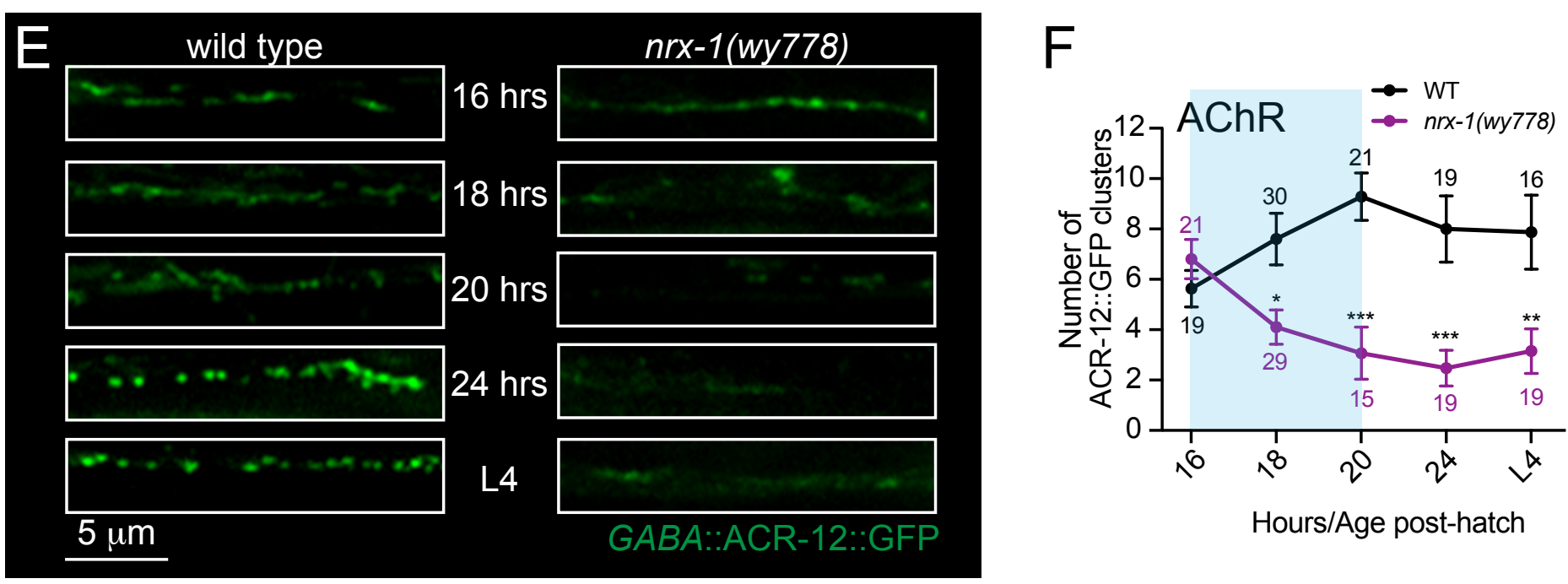

Hours/Age post-hatch

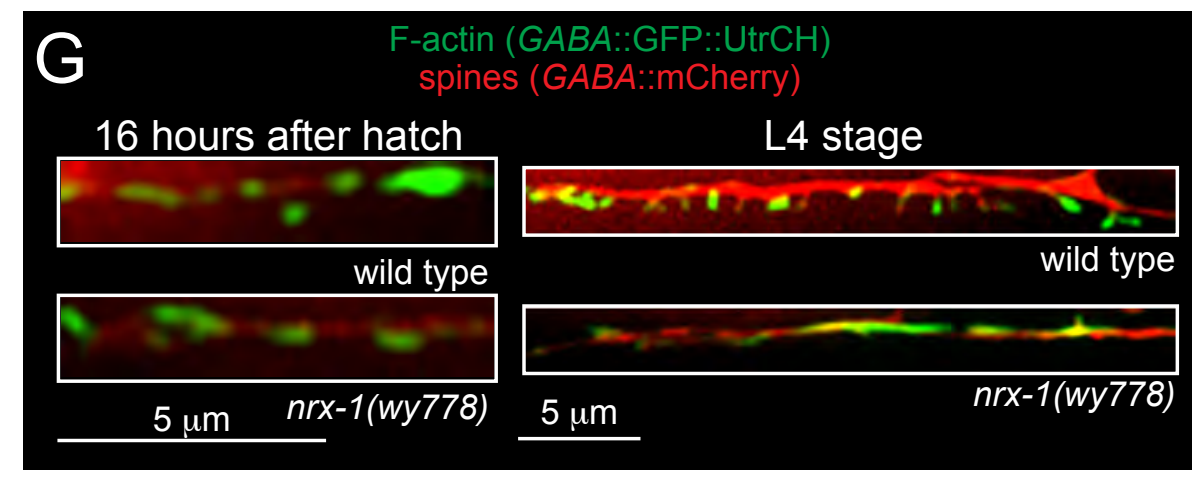


Figure 3. Dendritic spines and postsynaptic components form initially but subsequently collapse without the synaptic adhesion protein, NRX-1.

(A) Fluorescent confocal images of dendritic spines (Pflp-13::mCherry, magenta) and cholinergic expression of NRX-1::GFP (Punc-17 $\beta:$ NRX-1::GFP, cyan) at 16, 20, and 24 hours after hatch and L4 stage ( 42-50 hours post-hatch). Presynaptic clusters of NRX$1::$ GFP are visible by 16 hours after hatch and increase in size until roughly 24 hours after hatch.

(B) Quantification of the average number of spines (purple) and NRX-1 clusters (black) at the time points indicated. Blue shading indicates the approximate period of DD remodeling. Numbers indicate the number of animals quantified at each timepoint. Data points indicate mean \pm SEM.

(C) Fluorescent images (inverted LUT) of DD dendritic spines (Pflp-13::myrGFP) in wild type (left) and $n r x-1$ (wy778) (right) animals at 16, 18, 20, and 24 hours after hatch and at L4 stage. Immature dendritic spines clusters form initially but then collapse in $n r x-1$ mutants.

(D) Quantification of the percentage of wild type (black) and $n r x-1$ (wy778) (purple) animals with 2 or more DD dendritic spines at the timepoints indicated. Numbers indicate the number of animals quantified at each timepoint.

(E) Fluorescent images of ACR-12/AChR clusters (Pflp-13::ACR-12::GFP) in DD dendrites of wild type (left) and nrx-1(wy778) mutants (right) 16, 20, and 24 hours after hatch and at L4 stage ( 42-50 hours post-hatch). Immature AChR clusters initially form but then disperse in $n r x-1$ mutants.

(F) Quantification of the number of ACR-12::GFP clusters in DD dendrites of wild type (black) and $n r x-1$ (wy778) mutants (purple). Two-way ANOVA, Sidak's multiple comparisons, ${ }^{*} p<0.05,{ }^{* *} p<0.01,{ }^{* *} p<0.001$. 
bioRxiv preprint doi: https://doi.org/10.1101/2021.05.11.443328; this version posted June 24, 2021. The copyright holder for this preprint (which was not certified by peer review) is the author/funder. All rights reserved. No reuse allowed without permission.

(G) Fluorescent confocal images of dendritic spines (Pflp-13::mCherry) and F-actin (Pflp13::GFP::UtrCH) in DD dendrites of wild type and $n r x-1$ (wy778) animals at 16 hours after hatch and L4 stage. 

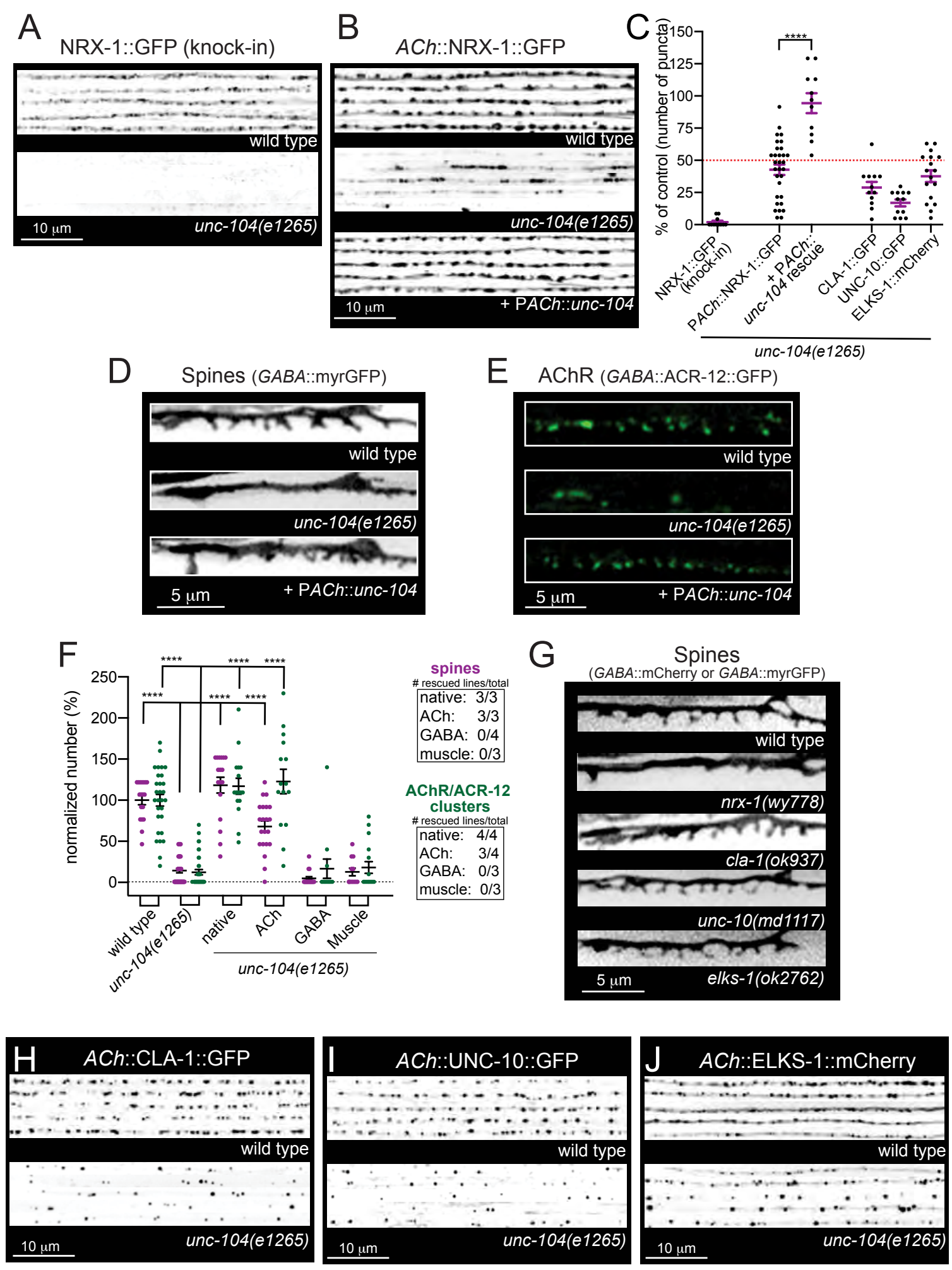
Figure 4. UNC-104/KIF1A motor-dependent transport of NRX-1 to presynaptic terminals is required for stabilization of postsynaptic structures.

(A) Representative confocal images of NRX-1::GFP (endogenous $n r x-1$ knock-in) from dorsal cords (inverted LUT) of wild type and unc-104(e1265) mutants. Images on each line are from different animals (5 shown for each genotype). Mutation of unc-104 impairs axonal NRX-1:GFP localization.

(B) Representative confocal images of cholinergic NRX-1::GFP (Punc-129::NRX-1::GFP, inverted LUT) from the dorsal nerve cord of wild type, unc-104(e1265) and unc-104(e1265) mutants rescued by cholinergic expression of wildtype unc-104 (Punc-17ß). Images on each line are from different animals (5 shown for each genotype).

(C) Scatterplot of the average number of NRX-1::GFP (endogenous or transgenic), CLA$1:: G F P$, UNC-10::GFP, or ELKS-1::mCherry clusters per $50 \mu \mathrm{m}$ of dorsal nerve cord in unc-104(e1265) mutants or rescue as indicated. Values are normalized to their respective controls. Red dotted line indicates $50 \%$ of wild type values. Bars indicate mean \pm SEM. Student's t-test, ${ }^{* * * *} p<0.0001 . \mathrm{n} \geq 11$ animals. Raw values with respective controls are shown in Figure S4.3.

(D) Fluorescent images (inverted LUT) of DD dendritic spines (Pflp-13::myrGFP) from wild type, unc-104(e1265), and unc-104(e1265) mutants rescued by cholinergic expression of wild type unc-104.

(E) Fluorescent images of AChR/ACR-12 clusters in DD dendrites (Pflp-13::ACR-12::GFP) of wild type, unc-104(e1265), and unc-104(e1265) mutants rescued by cholinergic expression of wild type unc-104.

(F) Scatterplot showing quantification of DD dendritic spines per $15 \mu \mathrm{m}$ (Pflp-13::myrGFP) (purple) and DD cholinergic receptor clusters (Pflp-13::ACR-12::GFP) (green) in wild type, unc-104(e1265), and indicated cell-specific rescue lines. Inset, number of rescuing 
lines/total transgenic lines tested for each rescue construct. Bars, mean \pm SEM. One-way ANOVA, Dunnett's multiple comparisons test, ${ }^{* * *} p<0.0001 . n \geq 10$ animals.

(G) Fluorescent images of DD dendritic spines (Pflp-13::myrGFP or Pflp-13::mCherry) from wild type, $n r x-1$ (wy778), cla-1(ok937), unc-10(md1117), or elks-1(ok2762) mutants. Only mutation of $n r x-1$ affects dendritic spines.

(H) Representative confocal images of cholinergic CLA-1 (Punc-17 $\beta$ :CLA-1::GFP, inverted LUT) from the dorsal nerve cord of wild type or unc-104(e1265) mutants. Images on each line are from different animals ( 5 shown for each genotype).

(I) Representative confocal images of cholinergic UNC-10 (Punc-129::UNC-10::GFP, inverted LUT) from dorsal nerve cords of wild type and unc-104(e1265) mutants. Images on each line are from different animals ( 5 shown for each genotype).

(J) Representative confocal images of cholinergic ELKS-1 (Punc-129::ELKS-1::mCherry, inverted LUT) from dorsal nerve cords of wild type and unc-104(e1265) mutants. Images on each line are from different animals (5 shown for each genotype). 


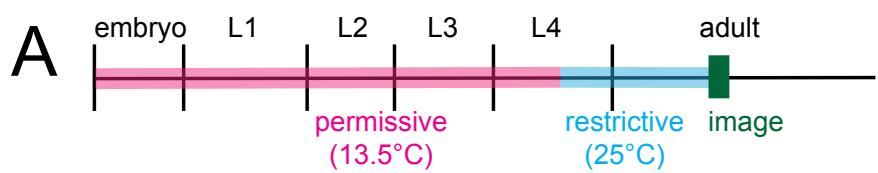

$\left(13.5^{\circ} \mathrm{C}\right)$
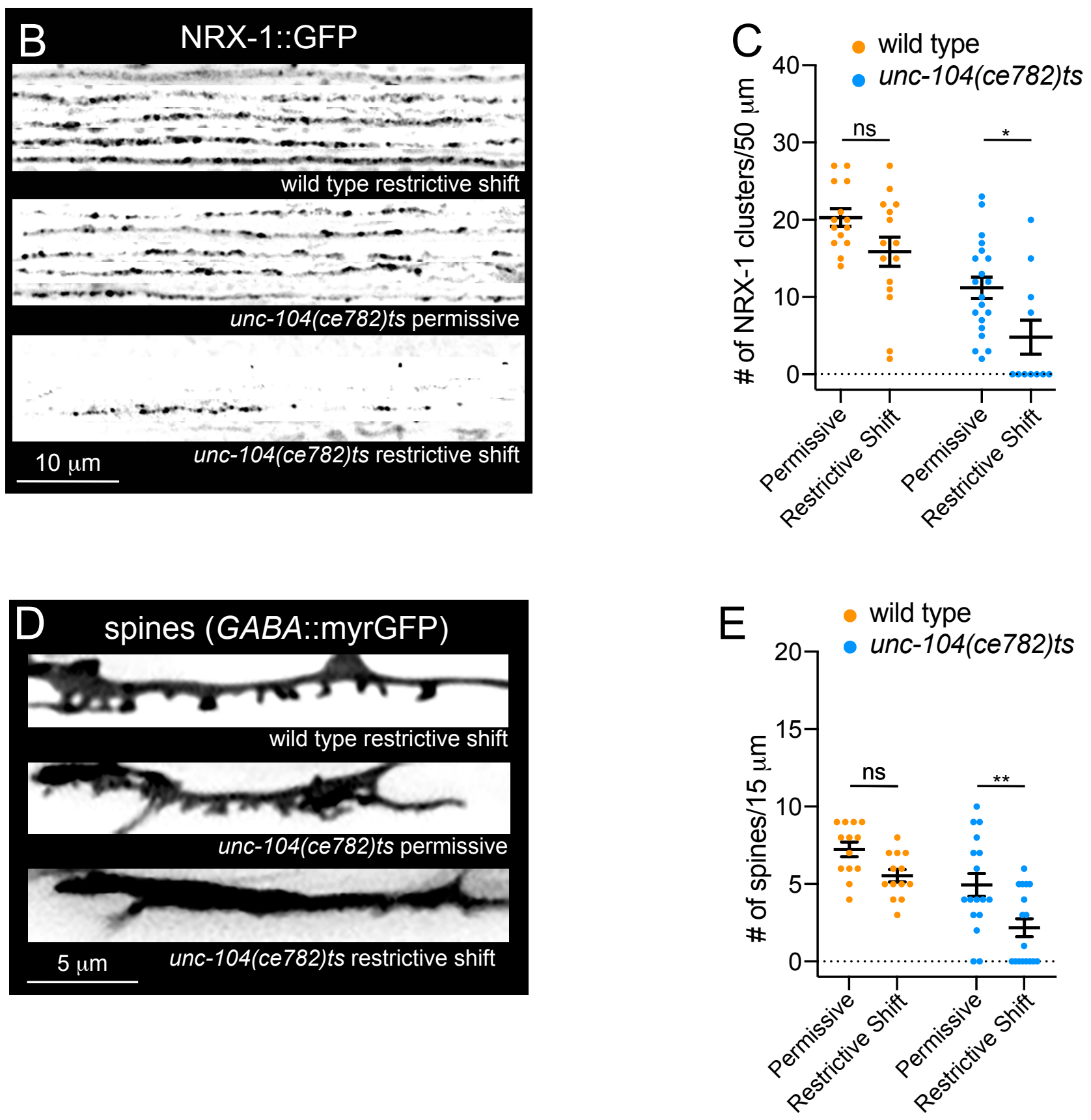
Figure 5. UNC-104/KIF1A transport of NRX-1 is required in the mature circuit to maintain postsynaptic structure.

(A) Cartoon depiction of experimental timeline. Animals were grown at $13.5^{\circ} \mathrm{C}$ until L4 stage (approx. 144 hours in unc-104(ce782)ts mutants, 120 hours in wild type animals) before shifting animals to the restrictive temperature of $25^{\circ} \mathrm{C}$ for $16-20$ hours and imaging.

(B) Line scan fluorescent images of NRX-1::GFP (knock-in to endogenous locus, inverted LUT) from dorsal cords of wild type and unc-104(ce782) mutants grown continuously at the permissive temperature $\left(13.5^{\circ} \mathrm{C}\right)$ (middle panel) or shifted to the restrictive temperature $\left(25^{\circ} \mathrm{C}\right)$ at L4 stage for $16-20$ hours before imaging (bottom panel). Each line scan indicates an individual animal.

(C) Quantification of NRX-1::GFP clusters along a $50 \mu \mathrm{m}$ region of the dorsal nerve cord in wild type and unc-104(ce782)ts animals. Two-way ANOVA, Tukey's multiple comparisons test, ns, not significant, ${ }^{*} p<0.05, \mathrm{n} \geq 11$ animals. Data points indicate mean \pm SEM.

(D) Fluorescent images of DD spines in wild type and unc-104(ce782)ts animals grown continuously at the permissive temperature $\left(13.5^{\circ} \mathrm{C}\right)$ (middle panel) or shifted to the restrictive temperature $\left(25^{\circ} \mathrm{C}\right)$ at $\mathrm{L} 4$ stage for $16-20$ hours before imaging.

(E) Quantification of the number of spines per $15 \mu \mathrm{m}$ in wild type and unc-104(ce782)ts animals. Two-Way ANOVA, Tukey's multiple comparisons test, ns, not significant, ${ }^{* *} p<0.001 . n \geq 13$ animals. Data points indicate mean \pm SEM. 
bioRxiv preprint doi: https://doi.org/10.1101/2021.05.11.443328; this version posted June 24, 2021. The copyright holder for this preprint (which was not certified by peer review) is the author/funder. All rights reserved. No reuse allowed without permission.

\section{Supplemental Figures}

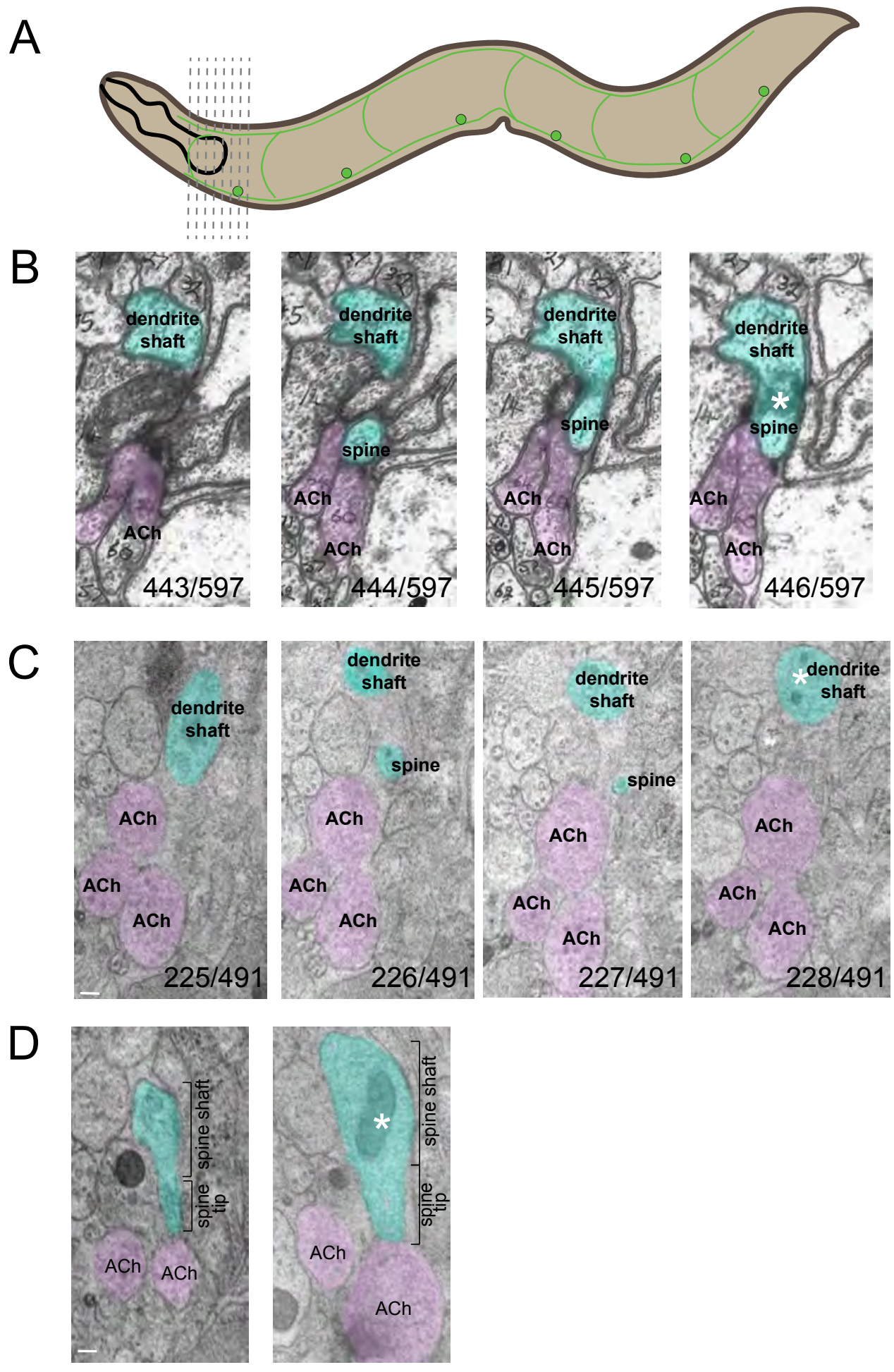




\section{Figure S1.1 Ultrastructure of DD dendritic spines.}

(A) Cartoon depiction of the anterior serial cross-sections used for electron microscopy studies of the ventral nerve cord, focusing on the dendrite of the DD1 neuron. Cross-sections in B are from White and colleagues (J. G. White et al., 1976) while sections in $C$ and $D$ are from this work.

(B) Serial cross-sections (443-446/597) of the ventral nerve cord from N2U series (J. G. White et al., 1976). Teal indicates DD1 dendrite and dendritic spines dipping into the ventral nerve cord to meet presynaptic cholinergic terminals (magenta). Asterisks indicates mitochondrion within the dendritic shaft, see Figure S1.4.

(C) Ventral nerve cord electron micrographs. Serial cross-sections (225-228/491) of the ventral nerve cord. Teal indicates DD1 dendrite and dendritic spines (226/491 and 227/491) dipping into the ventral nerve cord to meet presynaptic cholinergic terminals (presumably VA/VB neurons, magenta). Asterisks indicates mitochondria within the dendritic shaft, see Figure S1.4. Scale bar, $100 \mathrm{~nm}$.

(D) Two representative micrographs from serial sections where the entire extent of the spine is visible are shown. Asterisk indicates mitochondrion within the dendritic shaft. Scale bar, $100 \mathrm{~nm}$. 


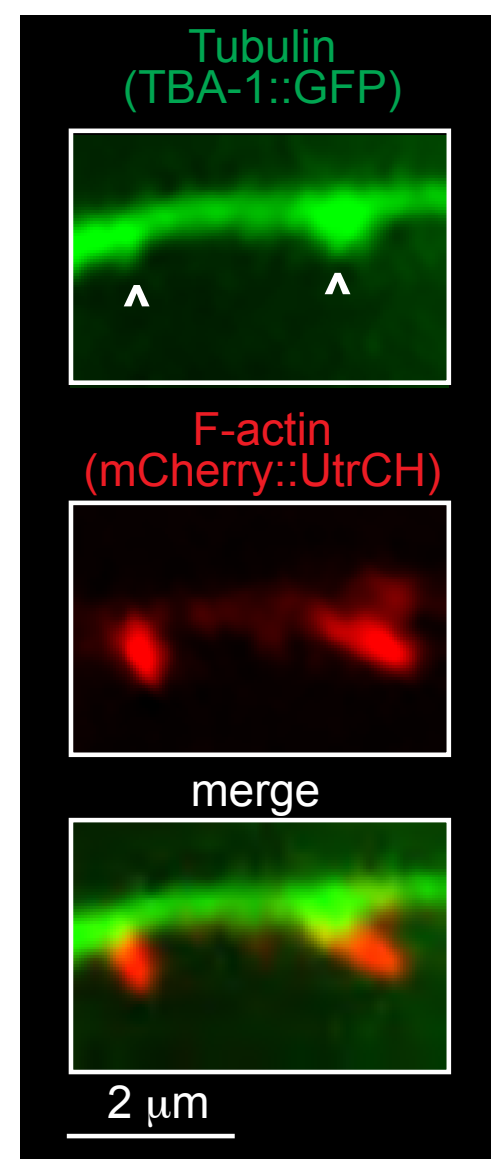

Figure S1.2. Microtubules localize at the base of spines.

Fluorescent images of tubulin (Pflp-13::TBA-1::GFP) and F-actin (Pflp-13::mCherry::UtrCH) in DD neurons. White arrows indicate dendritic regions where tubulin dips into the base of a dendritic spine. 


\section{8 nm laser}

Yokogawa CSU-X1-A1
spinning disk confocal

\section{(00000000000}

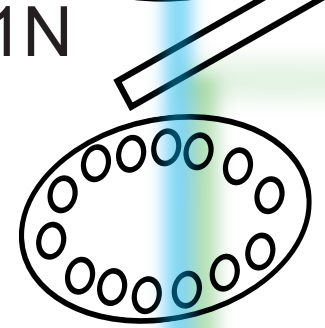

\section{AxiolmagerM2}

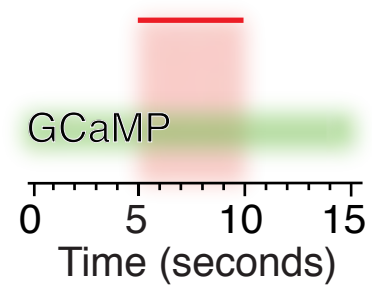

63X oil immersion

$625 \mathrm{~nm}$ LED 
Figure S1.3. Cartoon illustration of calcium imaging recording platform.

Imaging was carried out using a Yokogawa CSU-X1-A1N spinning disk confocal system (Perkin Elmer) equipped with EM-CCD camera (Hamamatsu, C9100-50) and 63X oil immersion objective. Chrimson photoactivation $\left(\sim 30 \mathrm{~mW} / \mathrm{cm}^{2}\right)$ was achieved using a TTL-controlled $625 \mathrm{~nm}$ light guide coupled LED (Mightex Systems), permitting illumination of the entire immobilized animal, while simultaneously recording GCaMP6f fluorescence (excitation $488 \mathrm{~nm}$, emission $525 \mathrm{~nm}$ ). A 556 $\mathrm{nm}$ edge BrightLine single-edge short-pass dichroic beam splitter was positioned in the light path (Semrock) to prevent $625 \mathrm{~nm}$ light from reaching the camera. 

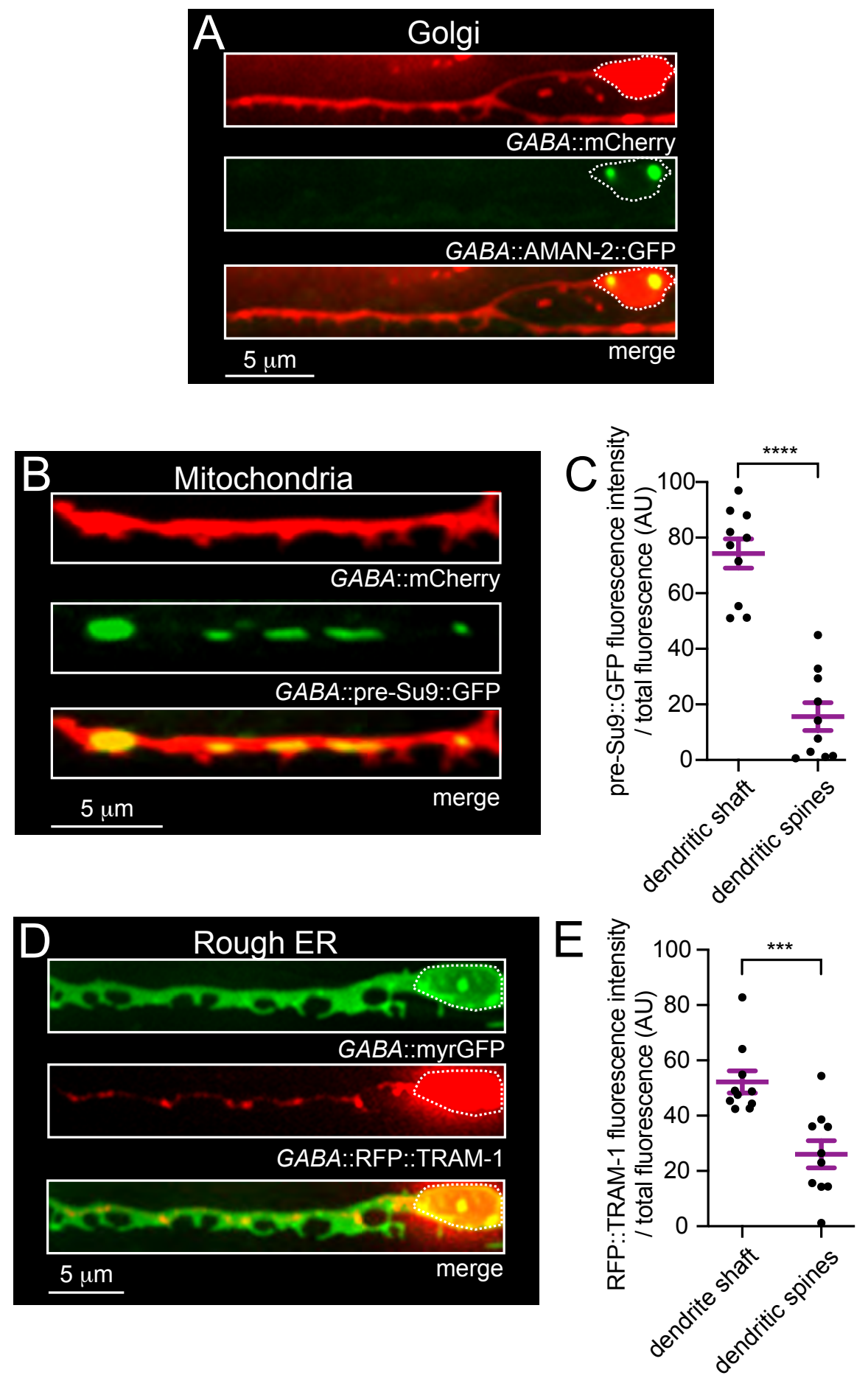
Figure S1.4. Examination of fluorescent organelle markers in DD dendrites and dendritic spines.

(A) Fluorescent images of DD1 dendritic spines (Pflp-13::mCherry) and Golgi marker (Pflp13::AMAN-2::GFP) in DD neurons. Golgi fluorescence is primarily restricted to the DD1 soma. Dotted white line traces the neuronal cell body.

(B) Fluorescent images of DD1 dendritic spines (Pflp-13::mCherry) and mitochondria (Pflp13::pre-Su9::GFP). Mitochondrial fluorescence is visible in the main dendritic processes of DD neurons near spines.

(C) Quantification of mitochondria fluorescence intensity in dendritic shaft and dendritic spines. Bars, mean \pm SEM. Student's t-test, ${ }^{* * *} p<0.0001$.

(D) Fluorescent images of DD1 dendritic spines (Pflp-13::myrGFP) and rough endoplasmic reticulum (ER) (Pflp-13::RFP::TRAM). Dotted white line traces the neuronal cell body. ER fluorescence is primarily observed in the main dendritic processes of DD neurons and occasionally at the spine base.

(E) Quantification of rough ER fluorescence intensity (Pflp-13::RFP::TRAM-1) in dendritic shaft and dendritic spines. Bars, mean \pm SEM. Student's t-test, ${ }^{* * *} p<0.001 . \mathrm{n}=9$ animals. 
neuron reporter

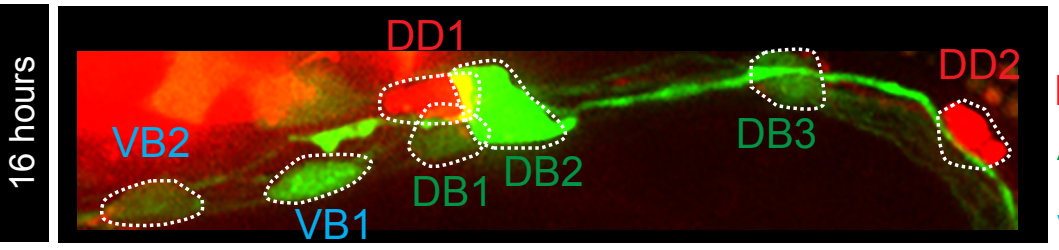

DD neurons:::mCherry (Pflp-13::mCherry)

ACh::GFP (Pacr-5::GFP)

post-embryonic born

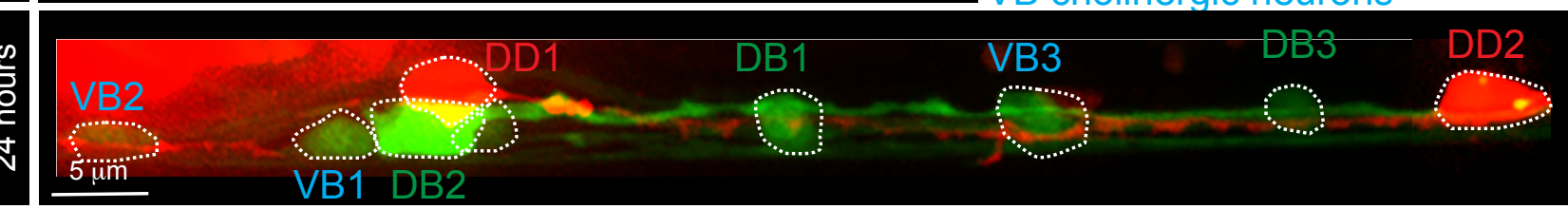

B

\section{synaptic vesicles}

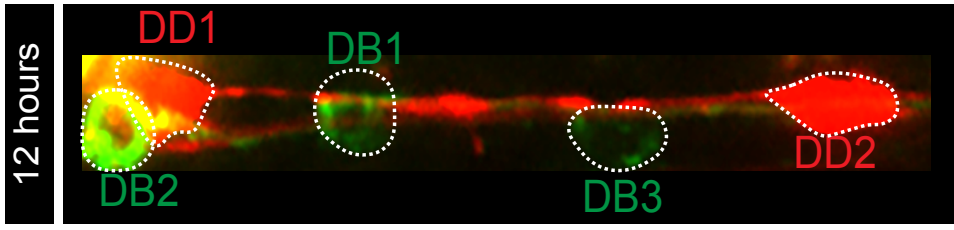

DD neurons::mCherry (Pflp-13::mCherry) ACh synaptic vesicles::GFP (Pacr-5::SNB-1::GFP) post-embryonic born VB cholinergic neurons
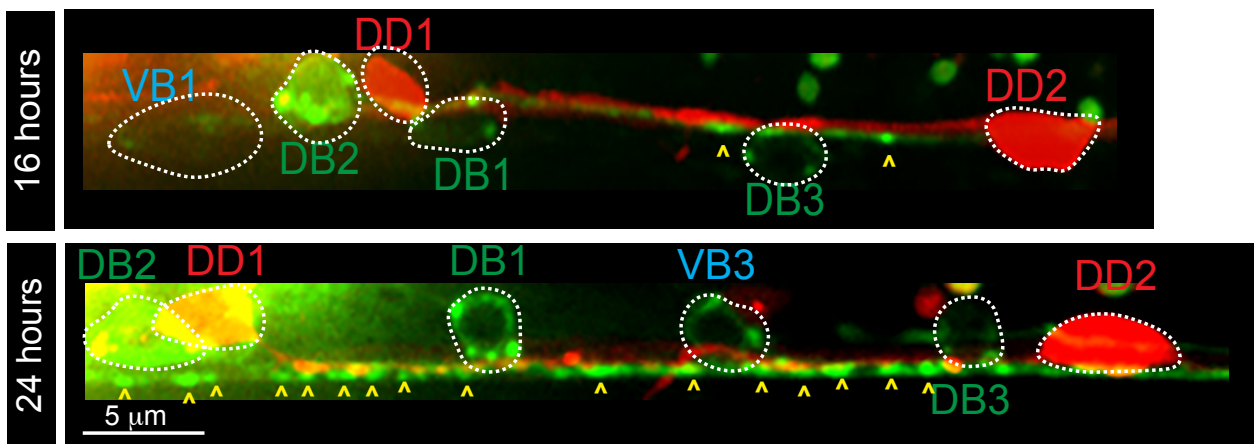

C

12 hours after hatch

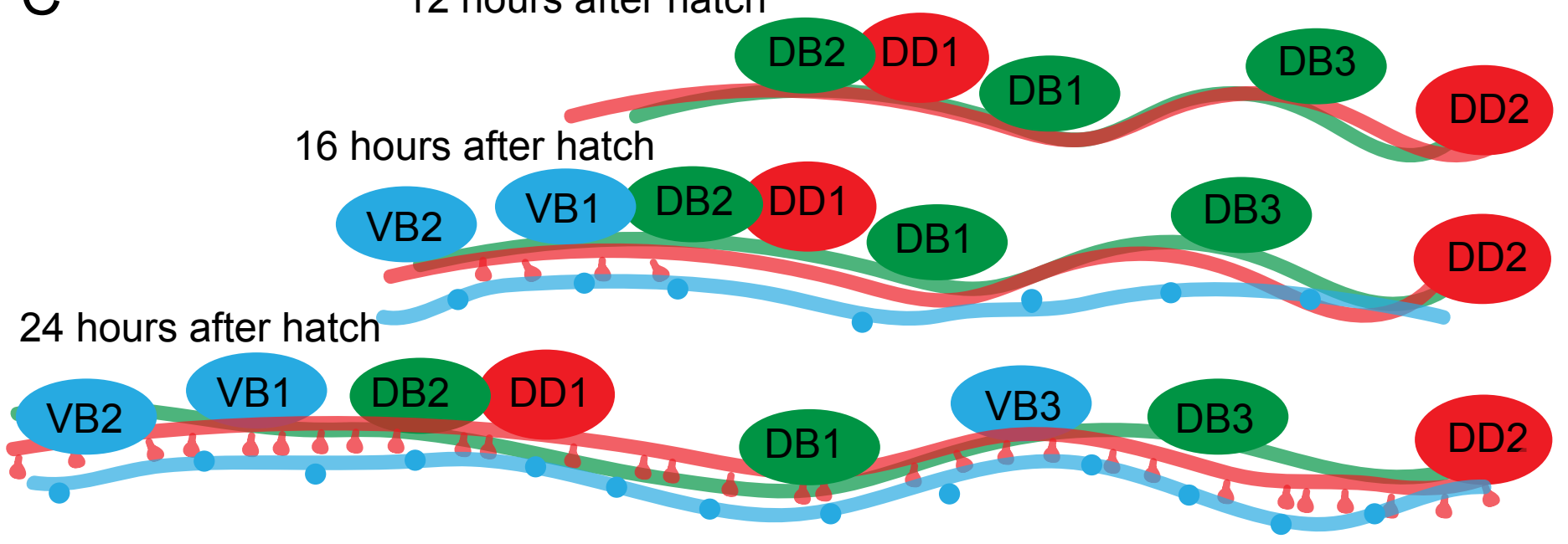

24 hours after hatch

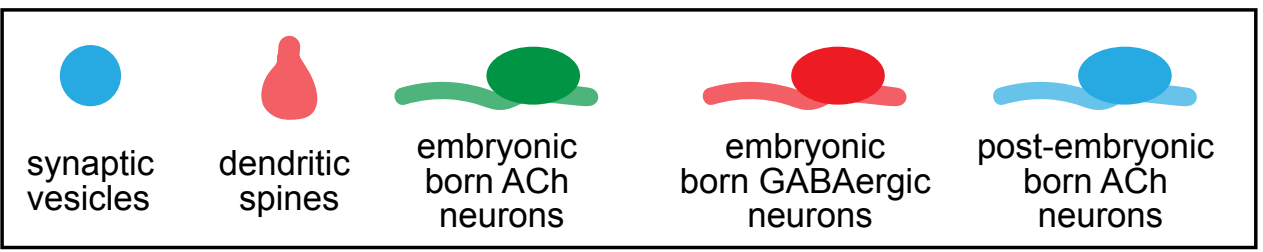


Figure S2.1. Development of post-embryonic born ventral cholinergic motor neurons.

(A) Fluorescent images of B-type cholinergic neurons (DB/VB) (Pacr-5::GFP) (green) and DD GABAergic neurons (Pflp-13::mCherry) (red) at 16 and 24 hours after hatch. VB cholinergic neurons are born post-embryonically in an anterior to posterior order (blue). Note that at 16 hours after hatch Pacr-5::GFP fluorescence indicating VB1 and VB2 cell bodies is visible. By 24 hours after hatch Pacr-5::GFP fluorescence indicating VB3 is visible. White dotted circles outline the neuronal cell bodies.

(B) Fluorescent images of the anterior ventral nerve cord in animals co-expressing the synaptic vesicle marker Pacr-5::SNB-1::GFP in B-type cholinergic neurons (DB/VB) (green) with Pflp-13::mCherry labeling DD GABAergic neurons (red) at 12, 16, and 24 hours after hatch. At 12 hours, little SNB-1::GFP fluorescence is visible in the ventral nerve cord. Embryonic born, dorsally directed B-type (DB) cholinergic motor neurons predominantly make synaptic contacts in the dorsal nerve cord. Ventrally-directed, B-type (VB) cholinergic motor neurons are born post-embryonically and have not yet completed their maturation at this time. SNB-1::GFP fluorescence in the ventral nerve cord is faintly visible by $16 \mathrm{hrs}$ after hatch and becomes more prominent by $24 \mathrm{hrs}$ after hatch, coincident with maturation of VB motor neurons. White dotted circles indicate outlines of neuronal cell bodies. Yellow arrows indicated presynaptic vesicle clusters, SNB-1.

(C) Cartoon representation of the developmental timing of post-embryonic born ventral cholinergic motor neurons, spine outgrowth, and cholinergic synaptic vesicle localization. 

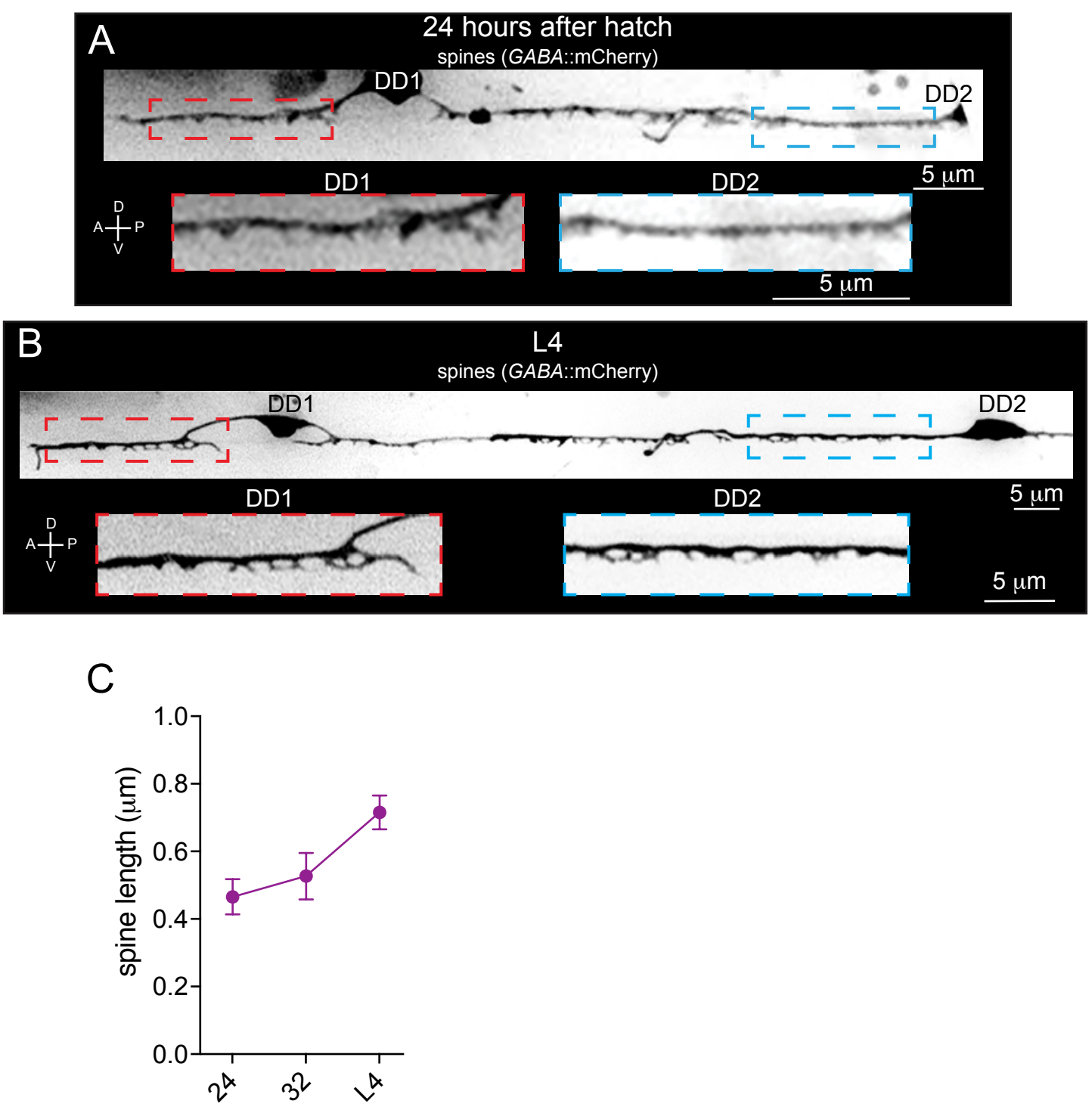

Hours/Age post-hatch 
Figure S2.2. Spine outgrowth progresses anteriorly to posteriorly.

(A and B) Fluorescent images (inverted LUT) of DD1 and DD2 dendritic spines 24 hours after hatch $(A)$ and at L4 ( 42-50 hours after hatch) stage (B). Animals express Pflp-13::mCherry to label dendritic spines. Red dashed rectangle indicates inset anterior to DD1 soma. Blue dashed rectangle indicates inset anterior to DD2 soma. Note that DD1 spines form prior to DD2 spines.

(C) Quantification of the length of growing dendritic spines at 24 and 32 hours after hatch and at L4 ( 42-50 hours after hatch) stage. Data points indicate mean \pm SEM. 

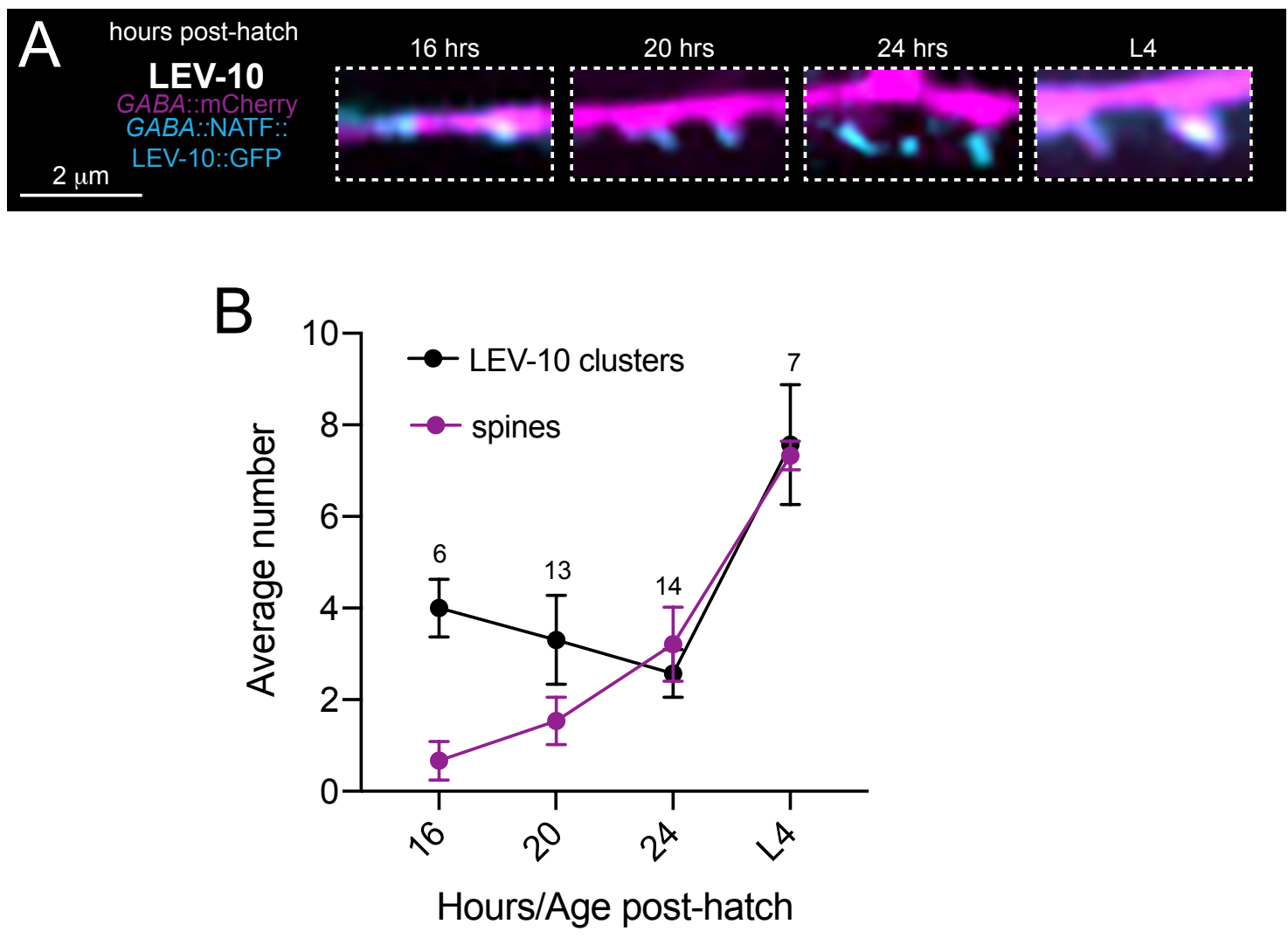
Figure S2.3. LEV-10 localizes to putative postsynaptic sites prior to spine formation.

(A) Fluorescent confocal images showing dendritic spines (magenta) and the postsynaptic CUB transmembrane domain LEV-10 (cyan). Animals express Pflp-13::mCherry with DD neuron specific LEV-10::GFP11 x7 using a strategy for cell-specific labeling of endogenous LEV-10 (NATF) (He et al., 2019).

(B) Quantification of the number of DD dendritic spines and LEV-10 clusters at 16, 20, and 24 hours after hatch, and L4 stage ( $\sim 2-50$ hours post-hatch). 
bioRxiv preprint doi: https://doi.org/10.1101/2021.05.11.443328; this version posted June 24, 2021. The copyright holder for this preprint (which was not certified by peer review) is the author/funder. All rights reserved. No reuse allowed without permission.
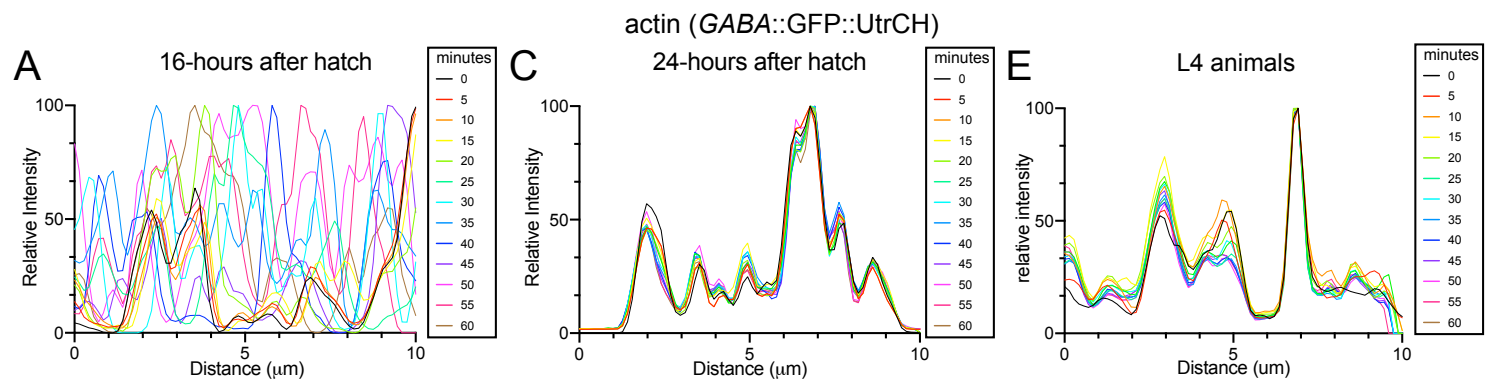

B

16-hours after hatch

D

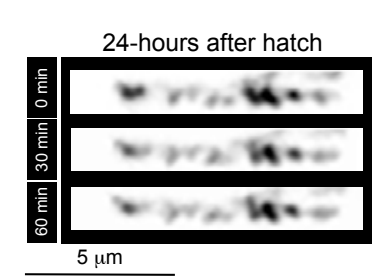

F

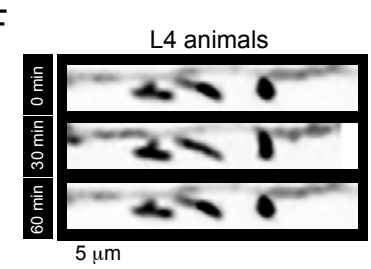

$5 \mu \mathrm{m}$

$5 \mu \mathrm{m}$

$5 \mu \mathrm{m}$ 
Figure S2.4. Actin dynamics during development.

$(A, C, E)$ Line scans displaying relative fluorescence intensity of F-actin (Pflp-13::GFP::UtrCH) at 16 hours after hatch $(A), 24$ hours after hatch $(C)$, and L4 ( 42-50 hours after hatch) animals (E). Each color indicates a line scan of fluorescence intensity for the same DD dendrite ROI acquired at 5-minute intervals. Note the variable distribution of fluorescence intensities across line scans from images acquired near 16 hours after hatch compared to later time points, indicating increased F-actin dynamics during early development.

$(\mathrm{B}, \mathrm{D}, \mathrm{F})$ Confocal images (inverted LUT) showing Pflp-13::GFP::UtrCH fluorescence (labeling Factin) in the DD dendrite of $16(B)$, or 24 (D) hours after hatch, or at L4 stage ( 42-50 hours after hatch) (F). For each, sequences of fluorescent images separated by 30 minutes are shown. 
Video 1: Confocal live imaging video of F-actin dynamics (labeled by Pflp-13::GFP::UtrCH) in the DD1 dendrite of wild type at 16 hours after hatch. For display, movies are shown at $3 \mathrm{fps}$. Images were acquired every 5 minutes.

Video 2: Confocal live imaging video of F-actin dynamics (labeled by Pflp-13::GFP::UtrCH) in the DD1 dendrite of wild type at L4 stage. For display, movies are shown at $3 \mathrm{fps}$. Images were acquired every 5 minutes. 

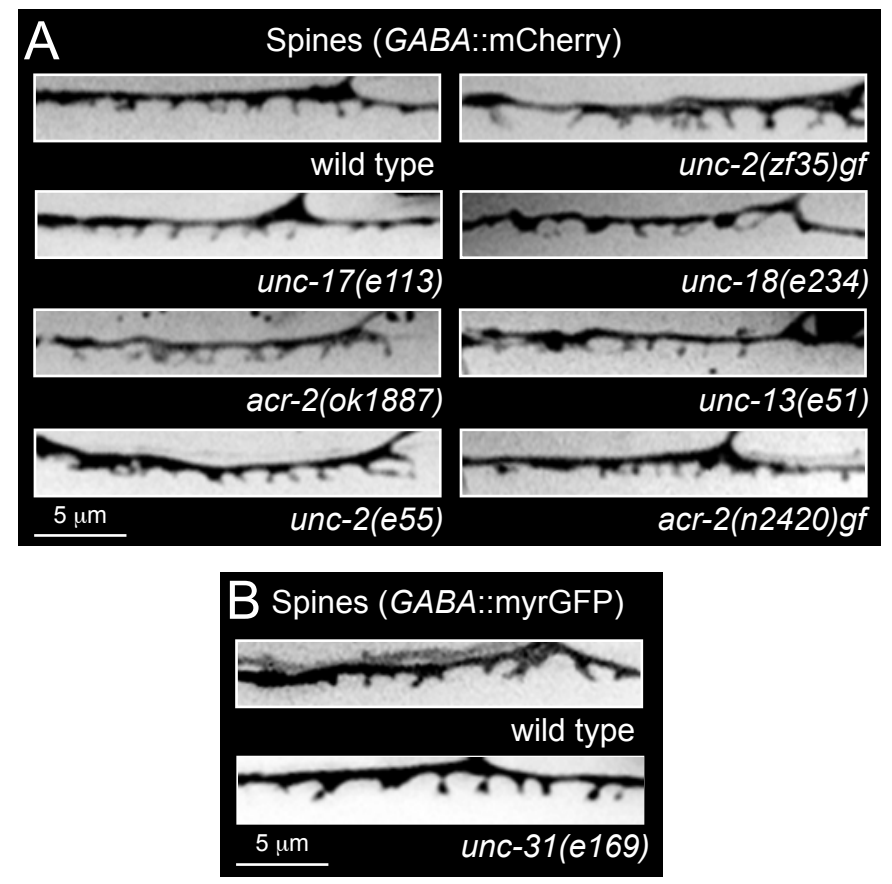

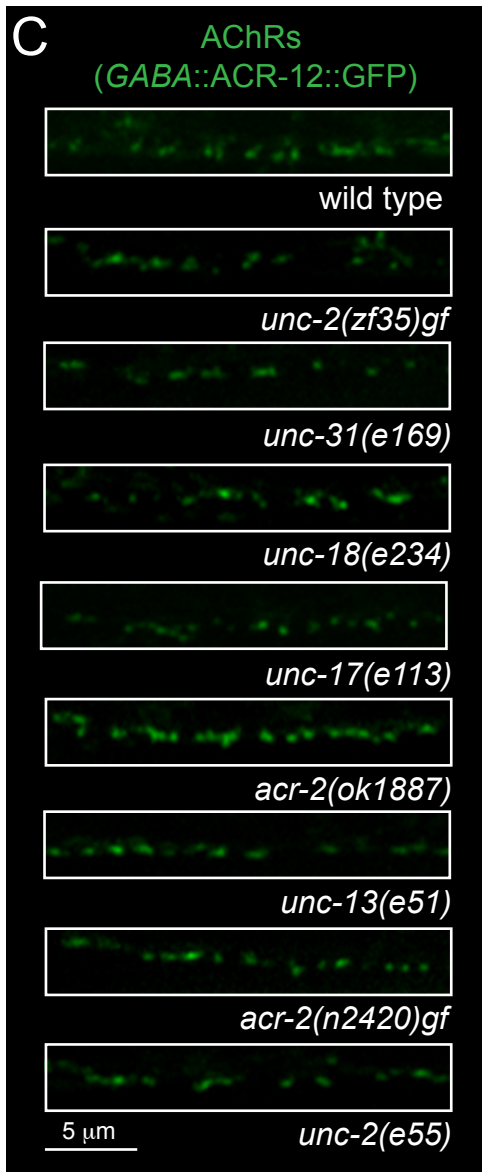


Figure S3.1. Spines and AChR localize independently of presynaptic activity.

$(A, B) \quad$ Fluorescent confocal images (inverted LUT) of DD1 dendritic spines (Pflp-13::mCherry (A) or Pflp-13::myrGFP (B)) in L4 stage wild type and selected mutant strains where synaptic activity is affected.

(C) Fluorescent confocal images of AChR clusters (Pflp-13::ACR-12::GFP) in the DD dendrite of L4 stage wild type and selected mutant strains where synaptic activity is affected. 


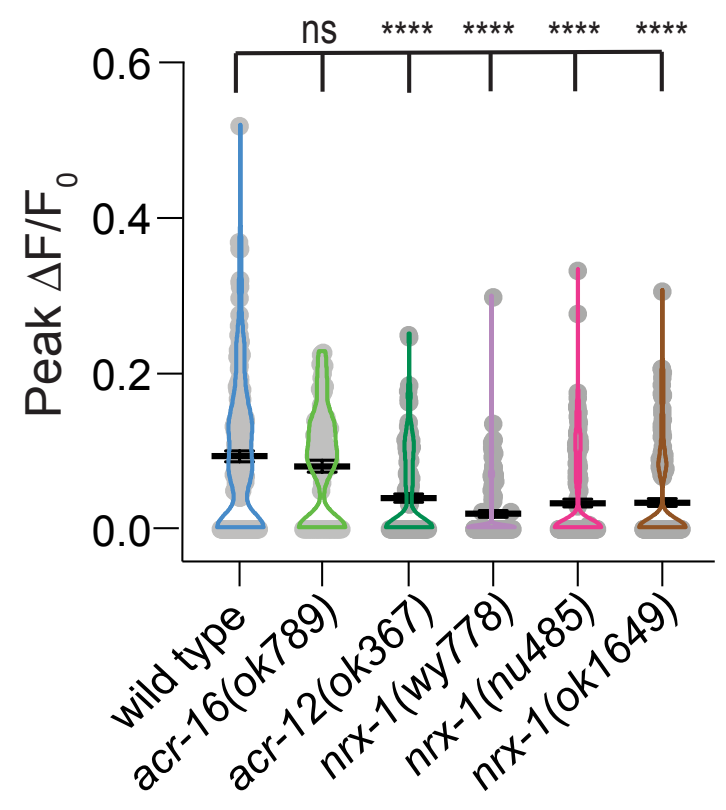


Figure S3.2. Dendritic calcium responses are severely reduced in nrx-1 mutants.

Scatter plot showing peak $\Delta F / F_{0}$ responses measured from GABAergic DD motor neuron dendrites during a 5 s period of cholinergic photostimulation in wild type, acr-16(ok789); acr12(ok367), nrx-1(wy778), nrx-1(nu485), and nrx-1(ok1649) mutants. All genotypes co-express Pflp-13::myrGCaMP6f::SL2::mCherry for measurement of dendritic calcium responses with Pacr2::Chrimson for cholinergic neuron depolarization. Dendritic calcium responses were not significantly affected by deletion of the homomeric nAChR subunit acr-16, but were significantly reduced by mutation of either acr-12 or $n r x-1$. Bars indicate mean \pm SEM. One-way ANOVA, Dunnett's multiple comparisons, ${ }^{* * * *} p<0.0001$. Wild type control is the same as Figure $1 \mathrm{~J} . \mathrm{n} \geq 10$ animals. 
A

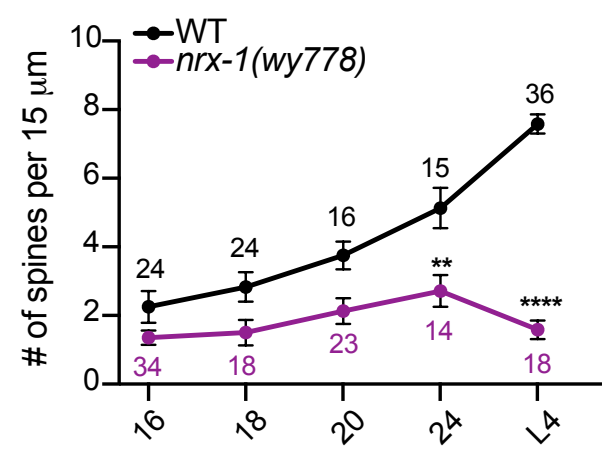

Hours post-hatch

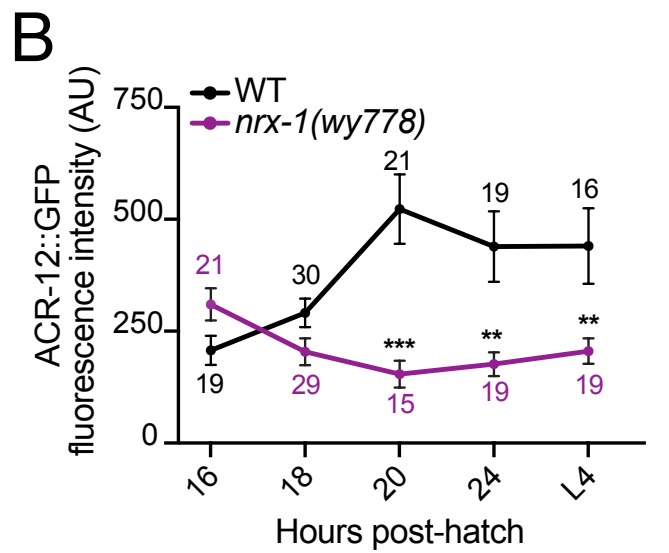


Figure S3.3. Spine and AChR localization in developing wild type and $n r x-1(w y 778)$ mutants.

(A) Quantification of the number of DD spines/15 $\mu \mathrm{m}$ in wild type (black) and $n r x-1$ (wy778) (purple) animals 16, 20, and 24 hours after hatch and at L4 stage ( $42-50$ hours after hatch). Two-way ANOVA, Sidak's multiple comparisons test, ${ }^{* *} p<0.01,{ }^{* * *} p<0.0001$. Data points indicate mean \pm SEM. Numbers indicate animals quantified for each timepoint.

(B) Quantification of ACR-12::GFP fluorescence intensity from DD dendrites of wild type (black) and $n r x-1$ (wy778) (purple) animals at 16, 20, and 24 hours after hatch and L4 stage ( 42-50 hours post-hatch). Two-way ANOVA, Sidak's multiple comparisons test, ${ }^{* *} p<0.01$, ${ }^{* * *} p<0.001$. Data points indicate mean \pm SEM. 
bioRxiv preprint doi: https://doi.org/10.1101/2021.05.11.443328; this version posted June 24, 2021. The copyright holder for this preprint (which was not certified by peer review) is the author/funder. All rights reserved. No reuse allowed without permission.

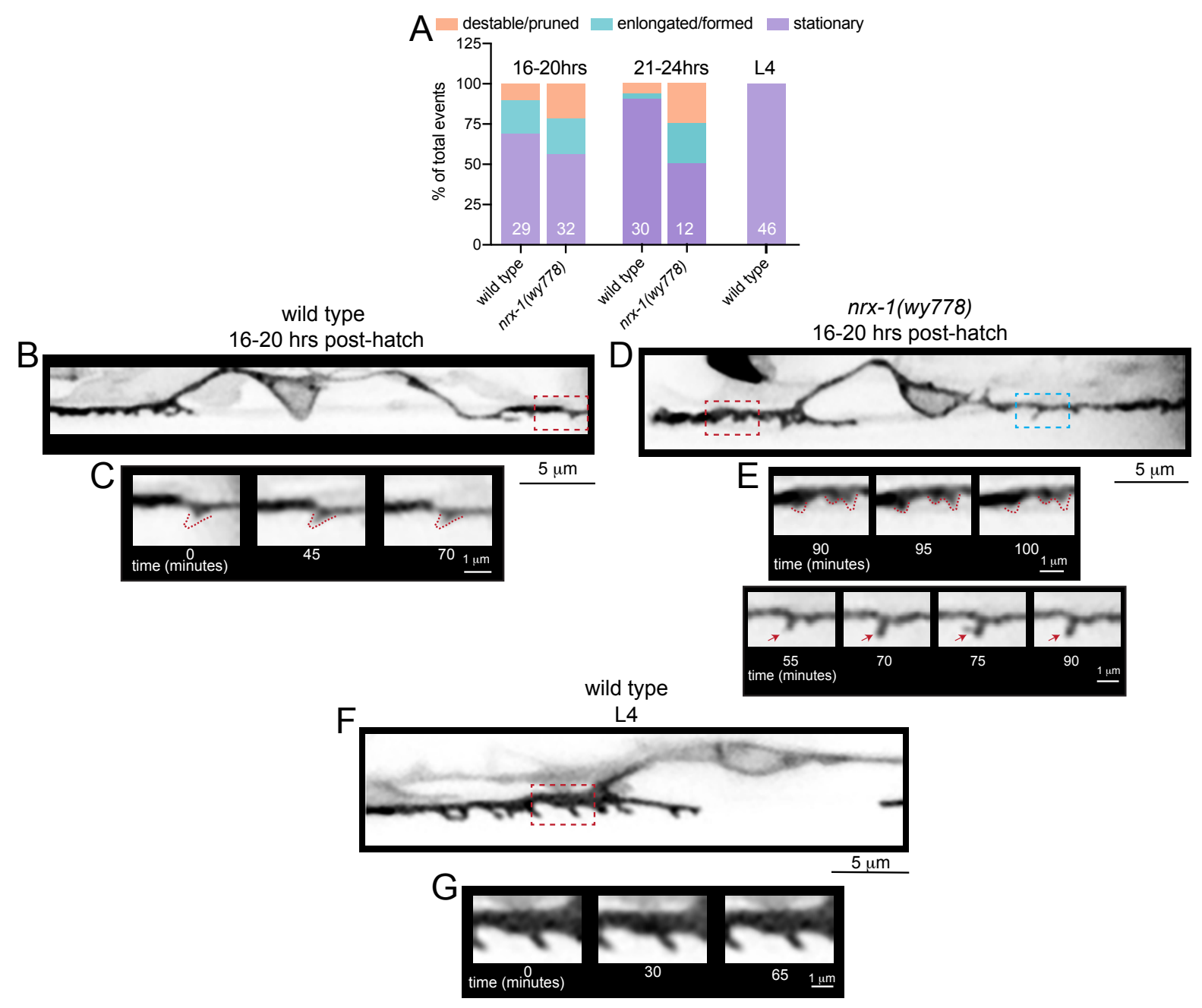


Figure S3.4. Spine dynamics during development in wild type and nrx-1(wy778) mutants.

(A) Quantification of the percentage of spine events in wild type and $n r x-1$ (wy778) animals at 16-20 hours after hatch, 21-24 hours after hatch, and at L4 ( 42-50 hours after hatch) compared to the total number of events. An event is defined as an incidence of spine destabilization/pruning, elongation/formation, or a stable spine during an entire live imaging recording. Z-stacks of DD1 dendrites were acquired every 5 minutes for $60-160$ minutes. Numbers indicates number of events for each genotype at each timepoint.

(B) Fluorescent image (inverted LUT) of DD dendritic spines at timepoint zero in a wild type animal 16-20 hours after hatch. Dashed red box indicates region shown in Figure S3.4C.

(C) Fluorescent images (inverted LUT) of an individual wild type DD dendritic spine (Pflp13::mCherry) at 0,45 , and 70 minute timepoints during live imaging of area indicated by red box in Figure S3.4B. Red dashed line indicates largest extent of spine outgrowth.

(D) Fluorescent image (inverted LUT) of $n r x-1$ (wy778) mutant DD1 dendritic spines (Pflp13::mCherry) at timepoint zero acquired 16-20 hours after hatch. Dashed red and blue boxes indicate regions shown in Figure S3.4E.

(E) Fluorescent images (inverted LUT) of individual $n r x-1$ (wy778) mutant DD dendritic spines (Pflp-13::mCherry) at (top) 90. 95, and 100 minute timepoints during live imaging of area indicated by red box in Figure S3.4D and (bottom) at 555, 70, 75, 90 minute timepoints during live imaging of area indicated by blue box in Figure S3.4D. Red dashed line indicates largest extent of spine outgrowth. Red arrows indicate spine dynamics.

(F) Fluorescent image (inverted LUT) of wild type DD dendritic spines (Pflp-13::mCherry) at timepoint zero at L4 stage. Dashed red box indicates region shown in Figure S3.4G.

(G) Fluorescent images (inverted LUT) of an individual wild type DD dendritic spine (Pflp13::mCherry) at 0,30 , and 65 minute timepoints during live imaging of the area indicated by red box in Figure S3.4F. 
Video 3. Wild type DD dendritic spines (Pflp-13::myrGFP) 16 hours after hatch. A z-stack was acquired every 5 minutes for at least $1 \mathrm{hr}$ of imaging duration. For display, movies are shown at $3 \mathrm{fps}$

Video 4. $n r x-1$ (wy778) mutant DD dendritic spines (Pflp-13::myrGFP) 16 hours after hatch. A zstack was acquired every 5 minutes for at least $1 \mathrm{hr}$ of imaging duration. For display, movies are shown at $3 \mathrm{fps}$

Video 5. Wild type DD dendritic spines (Pflp-13::myrGFP) at L4 stage. A z-stack was acquired every 5 minutes for at least $1 \mathrm{hr}$ of imaging duration. For display, movies are shown at 3 fps 

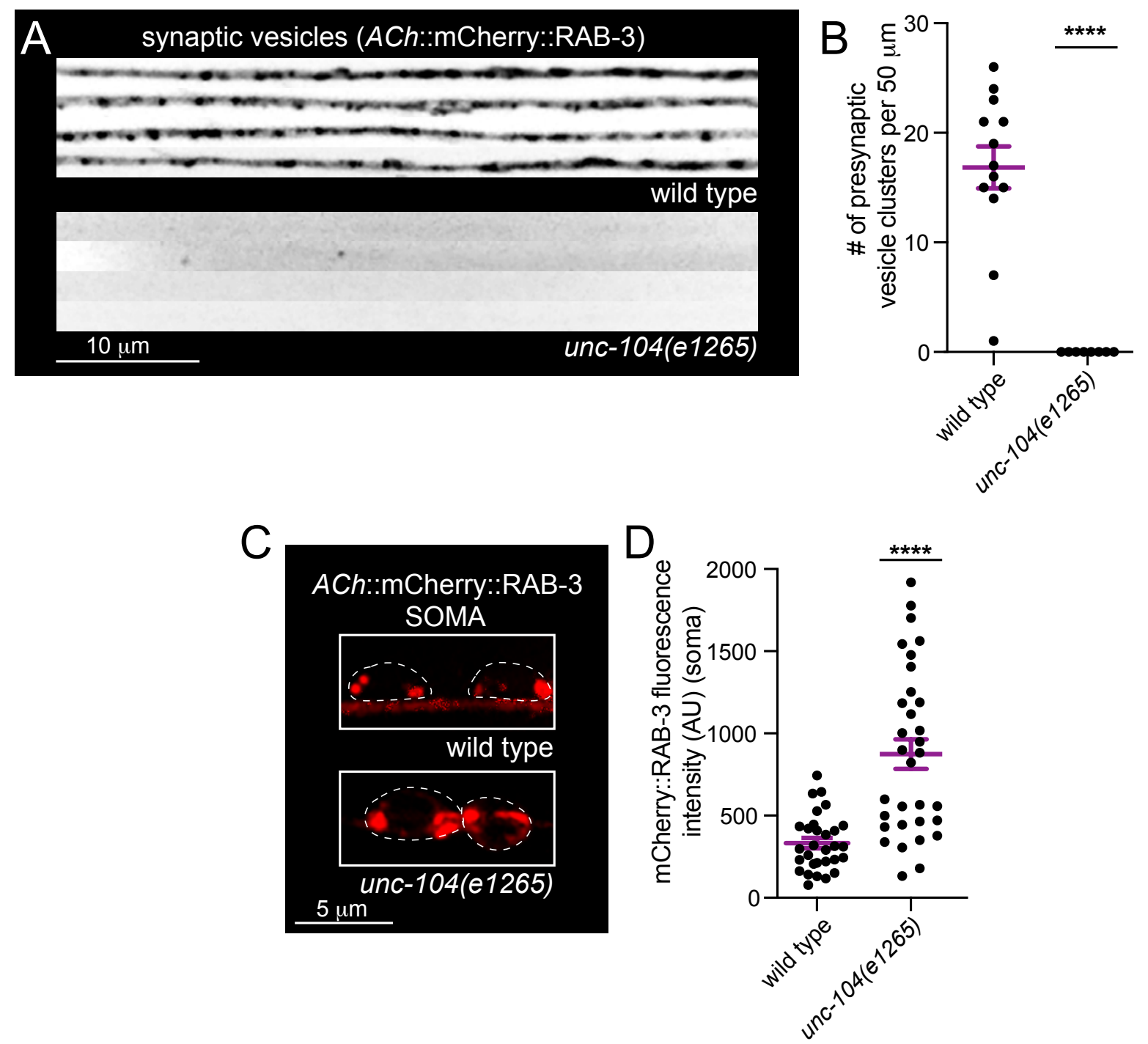
Figure S4.1. Mutation of unc-104 impairs synaptic vesicle delivery to cholinergic axons in the dorsal nerve cord.

(A) Fluorescent images of cholinergic synaptic vesicles (Pacr-2::mCherry::RAB-3) in the dorsal nerve cord of young adult wild type and unc-104(e1265) mutants. Images on each line are from different animals (4 are shown for each genotype).

(B) Quantification of cholinergic synaptic vesicles (Pacr-2::mCherry::RAB-3) per $50 \mu \mathrm{m}$ of the dorsal nerve cords of wild type and unc-104(e1265) mutants. Student's t-test, ${ }^{* * * *} p<0.0001$. Bars, mean \pm SEM.

(C) Fluorescent images of the soma in wild type and unc-104(e1265) mutants expressing cholinergic vesicle reporter (Pacr-2::mCherry::RAB-3). Dotted white lines outline the cell body.

(D) Quantification of soma cholinergic synaptic vesicle fluorescence intensity (AU) (Pacr2::mCherry::RAB-3) of wild type and unc-104(e1265) mutants. Student's t-test, ${ }^{* * * *} p<0.0001$. Bars, mean \pm SEM. 


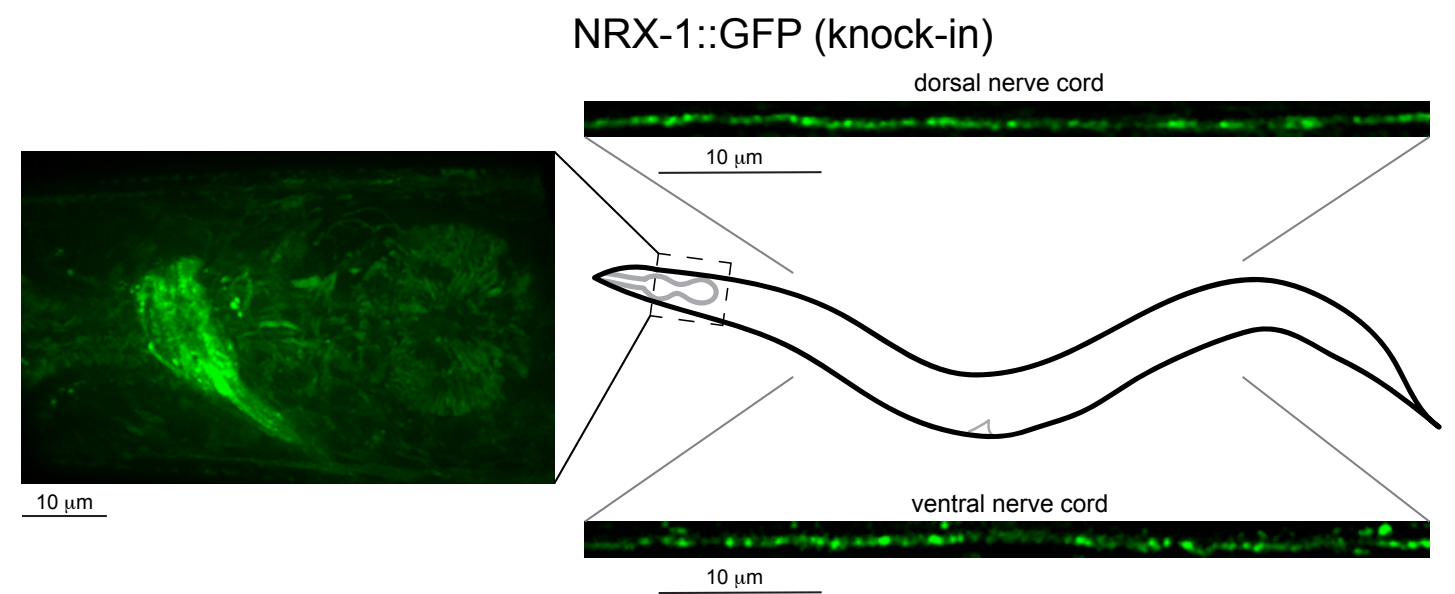

Figure S4.2. NRX-1 endogenously tagged with GFP localizes to neuronal processes.

NRX-1::GFP localizes within neuronal processes of the nerve ring and shows punctate localization in processes of the ventral and dorsal nerve cords of $L 4$ stage worms. 
NRX-1::GFP (knock-in)

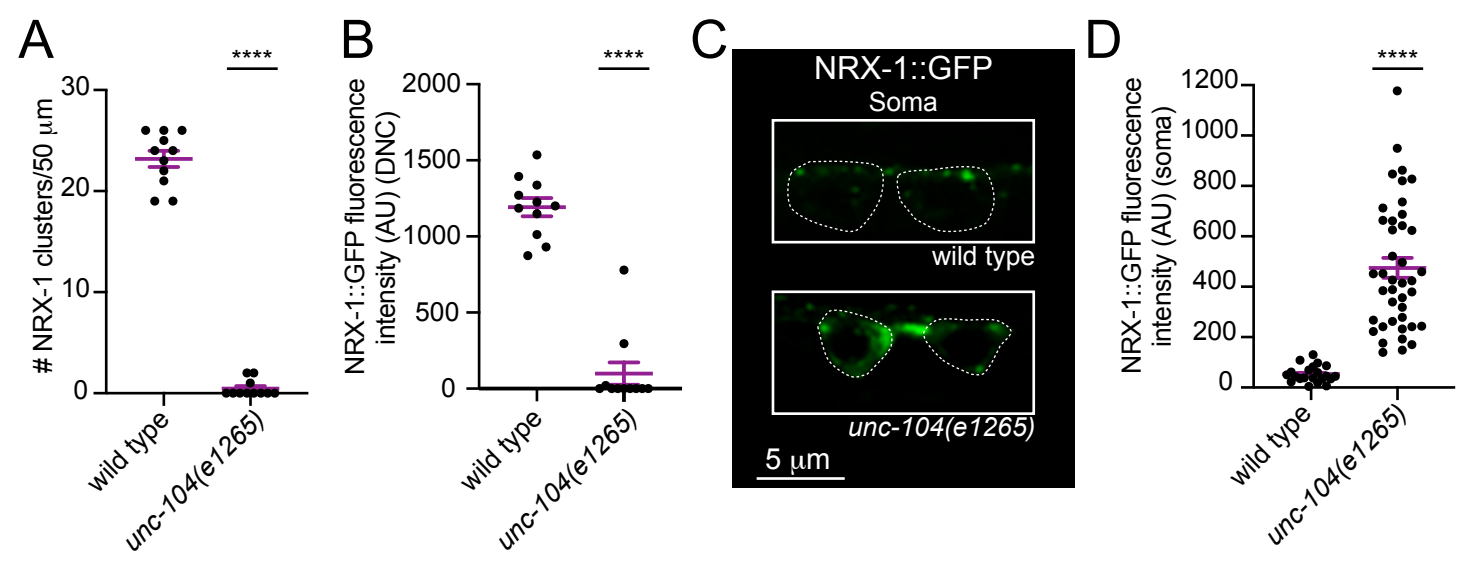

$A C h:: N R X-1:: G F P$

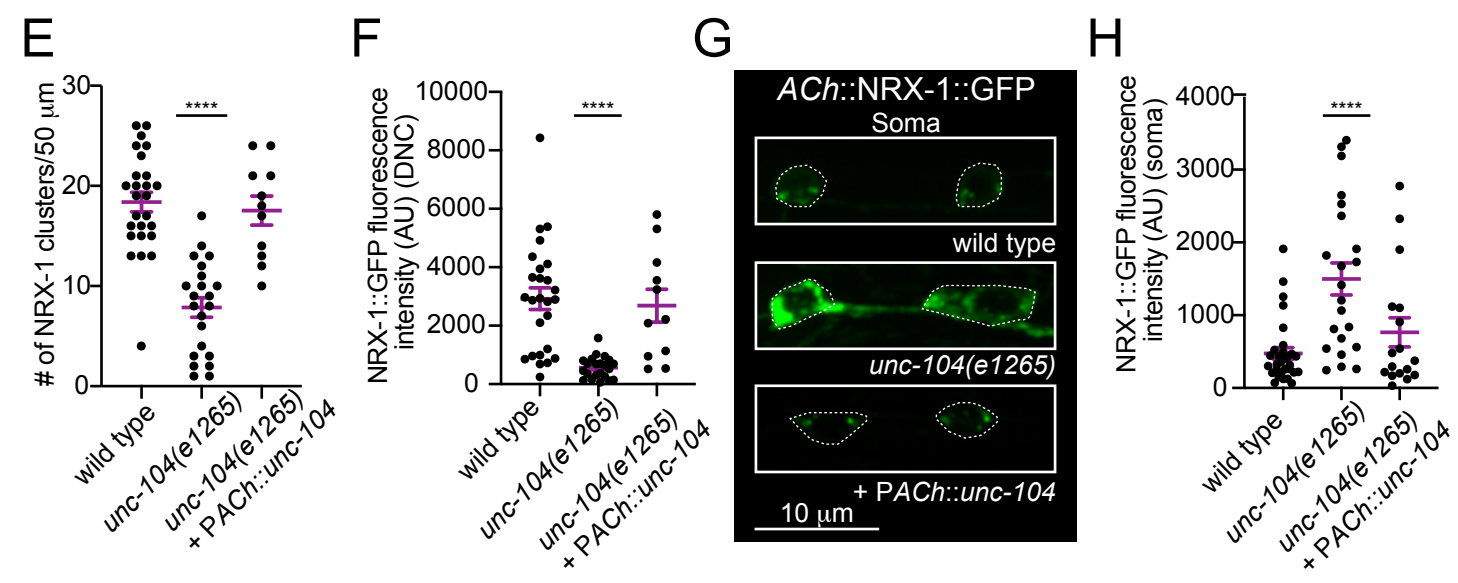


Figure S4.3. NRX-1 localization to cholinergic axons is decreased in unc-104 mutants.

(A) Quantification of NRX-1::GFP (endogenous knock-in) clusters in a $50 \mu \mathrm{m}$ region of the dorsal nerve cord in wild type and unc-104(e1265) animals. Bars, mean \pm SEM. Student's t-test, ${ }^{* * * *} p<0.0001 . \mathrm{n} \geq 11$ animals.

(B) Quantification of NRX-1::GFP (endogenous knock-in) axon fluorescence intensity in a $50 \mu \mathrm{m}$ region of the dorsal nerve cord. Bars, mean \pm SEM. Student's t-test, ${ }^{* * * *} p<0.0001 . \mathrm{n} \geq 11$ animals.

(C) Fluorescent images of NRX-1::GFP (endogenous knock-in) in somas of wild type and unc-104(e1265) mutants. Dotted white lines outline the neuronal cell body.

(D) Quantification of NRX-1::GFP soma fluorescence intensity. Bars, mean \pm SEM. Student's test, ${ }^{* * *} p<0.001$.

(E) Quantification of the number of NRX-1::GFP clusters in a $50 \mu \mathrm{m}$ region of the dorsal nerve cord in wild type, unc-104(e1265), and unc-104(e1265) mutants rescued with cholinergic expression of wild type unc-104. Bars, mean \pm SEM. One-way ANOVA, Dunnett's multiple comparisons test, ${ }^{* * *} p<0.0001 . \mathrm{n} \geq 11$ animals.

(F) Quantification of NRX-1::GFP fluorescence intensity in cholinergic axons of a $50 \mu \mathrm{m}$ region of the dorsal nerve cord. Bars, mean \pm SEM. One-way ANOVA, Dunnett's multiple comparisons test, ${ }^{* * * *} p<0.0001 . \mathrm{n} \geq 11$ animals.

(G) Fluorescent images of NRX-1::GFP in cholinergic somas of wild type, unc-104(e1265) and unc-104(e1265) mutants rescued with cholinergic expression of wild type unc-104. Dotted white lines outline the neuronal cell body.

(H) Quantification of NRX-1::GFP fluorescence intensity in cholinergic somas. Bars, mean \pm SEM. One-way ANOVA, Dunnett's multiple comparisons test, ${ }^{* * * *} p<0.001$. 


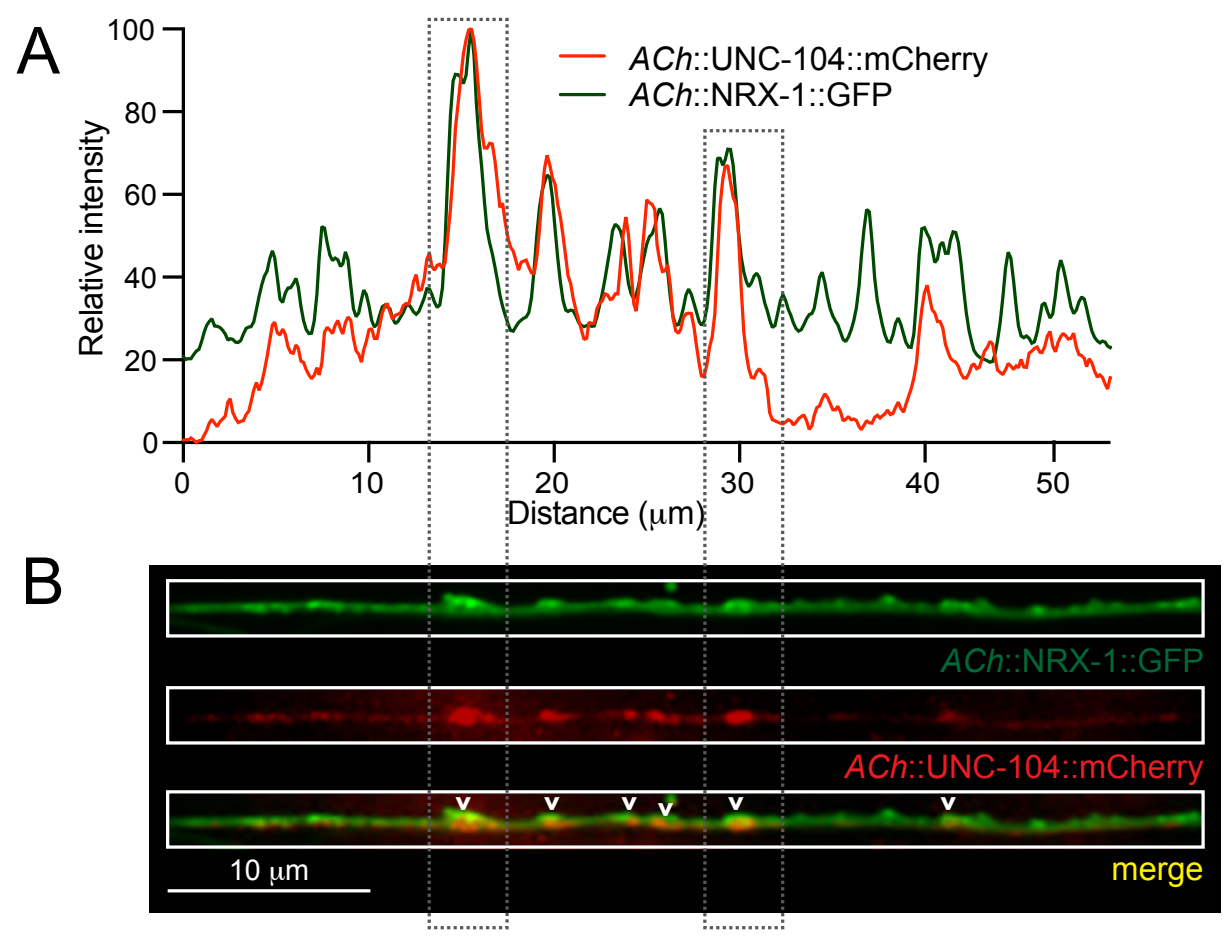


Figure S4.4. NRX-1 colocalizes with UNC-104 in cholinergic axon terminals.

(A) Line scans depicting relative fluorescent intensity of NRX-1::GFP (green) and UNC104::mCherry (red) for a $50 \mu \mathrm{m}$ region of the dorsal nerve cord. Gray dotted rectangles indicate corresponding puncta in Figure S4.4B.

(B) Fluorescent images of the dorsal nerve cord in an adult animal expressing NRX-1::GFP (Punc-129::NRX-1::GFP) and UNC-104::mCherry (Punc-129::UNC-104::mCherry). Colocalization is indicated by white carets. 

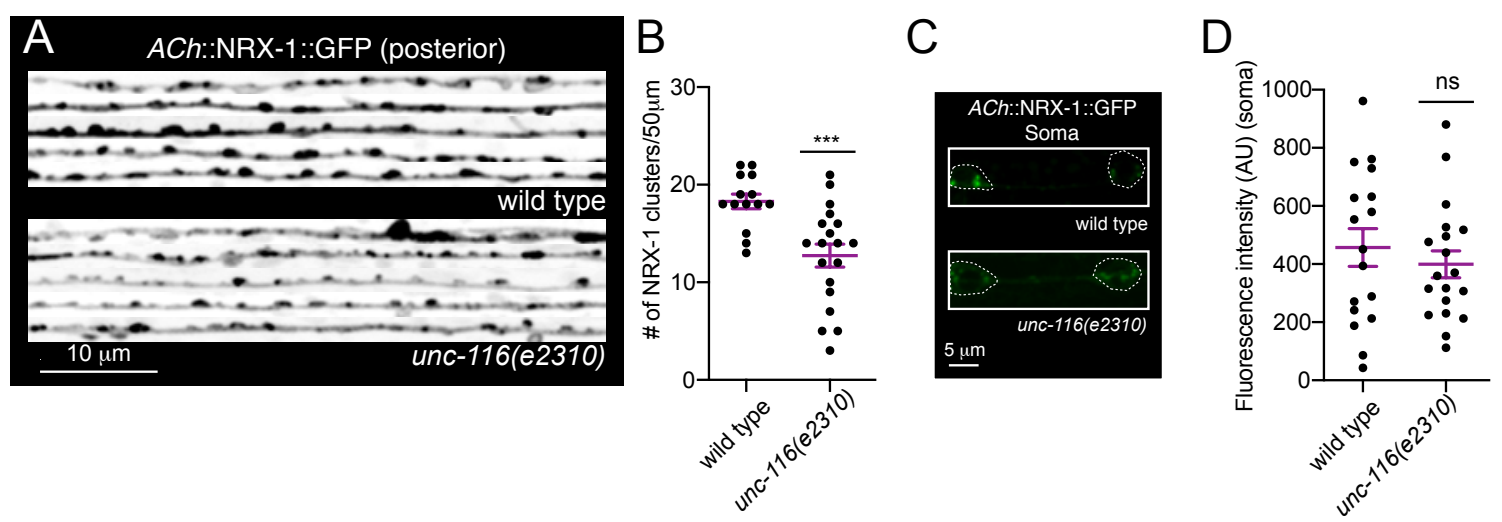

Figure S4.5. NRX-1 localization in unc-116/KIF5A/C mutants.

(A) Fluorescent images (inverted LUT) of NRX-1::GFP (Punc-129::NRX-1::GFP) from the dorsal nerve cord of wild type and unc-116(e2310) mutants. Images on each line are from different animals (5 are shown for each genotype).

(B) Quantification of the number of NRX-1 clusters (Punc-129::NRX-1::GFP) in a $50 \mu \mathrm{m}$ region of the dorsal nerve cord of wild type and unc-116(e2310) animals. Bars, mean \pm SEM. Student's t-test, ${ }^{* *} p<0.001 . \mathrm{n} \geq 14$ animals.

(C) Fluorescent images of NRX-1 (Punc-129::NRX-1::GFP) from cholinergic neuron somas of wild type and unc-116(e2310) mutants. Dotted white lines outline cell bodies.

(D) Quantification of fluorescence intensity of NRX-1 (Punc-129::NRX-1::GFP) from cholinergic somas of wild type and unc-116(e2310) mutants. Bars, mean \pm SEM. Student's t-test, ns, not significant. 


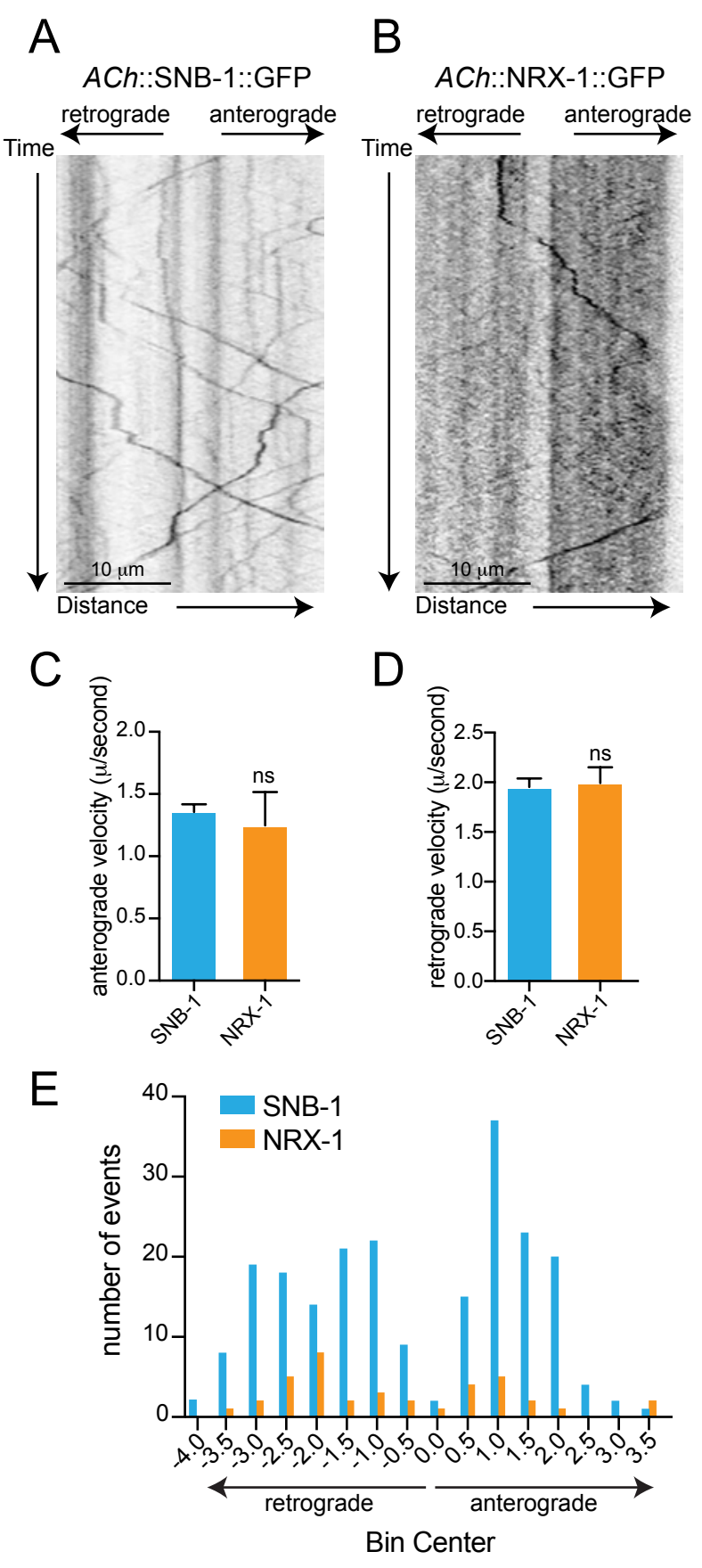


Figure S4.6. NRX-1 and SNB-1 have similar anterograde and retrograde velocities.

(A, B) Kymographs of synaptic vesicle (Punc-129::SNB-1::GFP) (A) and NRX-1 (Punc129::NRX-1::GFP) (B) trafficking events recorded from cholinergic neuron commissures.

(C) Quantification of the anterograde velocity ( $\mu \mathrm{m} /$ second) of SNB-1::GFP (blue) and NRX$1::$ GFP (orange) along the axonal commissure. $n s$, not significant. $n \geq 10$ animals for all panels.

(D) Quantification of the retrograde velocity ( $\mu \mathrm{m} /$ second) of SNB-1::GFP (blue) and NRX$1::$ GFP (orange) along the axonal commissure. ns, not significant.

(E) Quantification of the total number of SNB-1::GFP (blue) and NRX-1::GFP (orange) trafficking events binned into retrograde and anterograde directions. Note that NRX-1 trafficking events occur significantly less frequently than SNB-1 events. 
Video 6: NRX-1::GFP trafficking in a cholinergic commissure of a young adult animal (Punc129::NRX-1::GFP). Videos were recorded at $100 \mathrm{~ms}$ exposure for 30 seconds. For display, movie is shown at $25 \mathrm{fps}$.

Video 7: SNB-1::GFP trafficking in a cholinergic commissure of a young adult animal (Punc129::SNB-1::GFP). Videos were recorded at $100 \mathrm{~ms}$ exposure for 30 seconds. For display, movie is shown at $25 \mathrm{fps}$. 


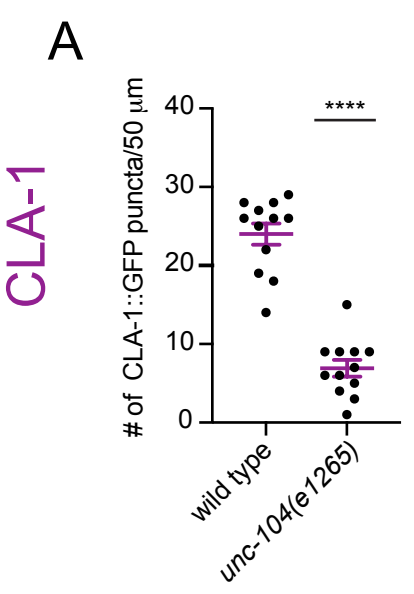

$\mathrm{B}$ 疋
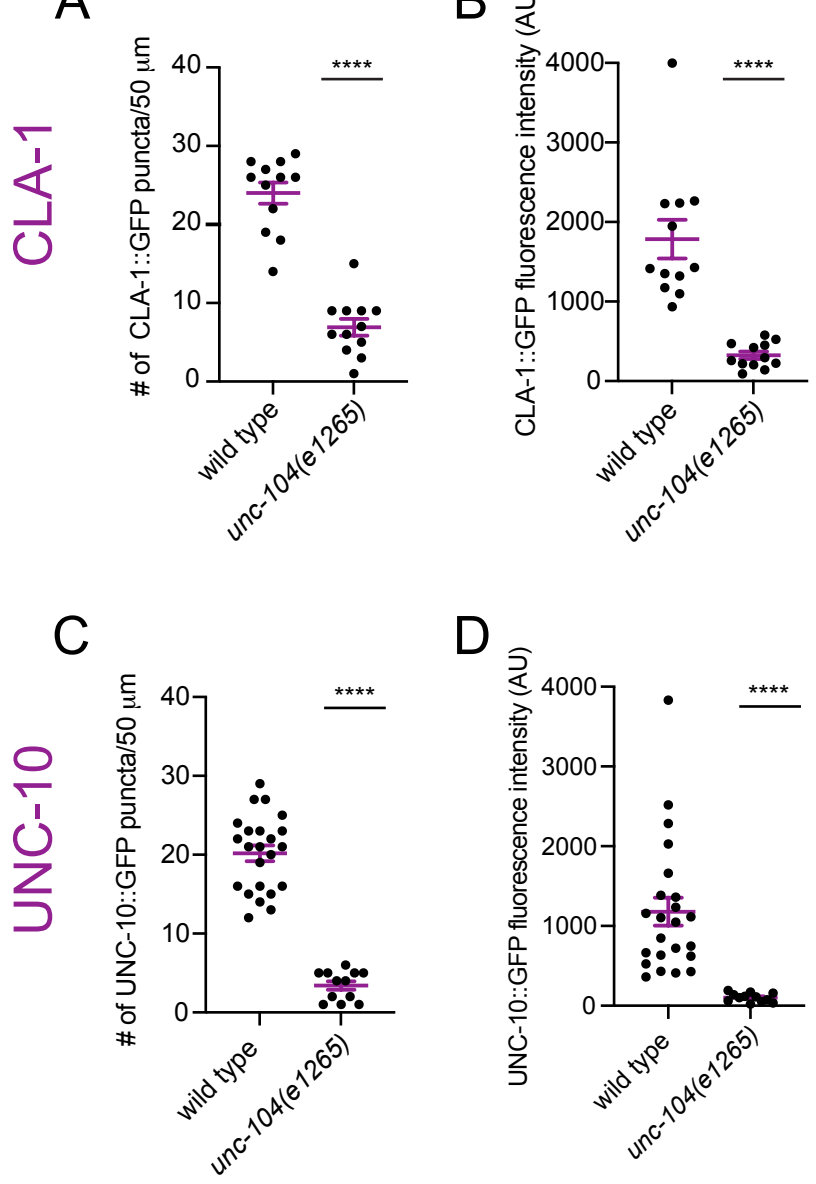

D
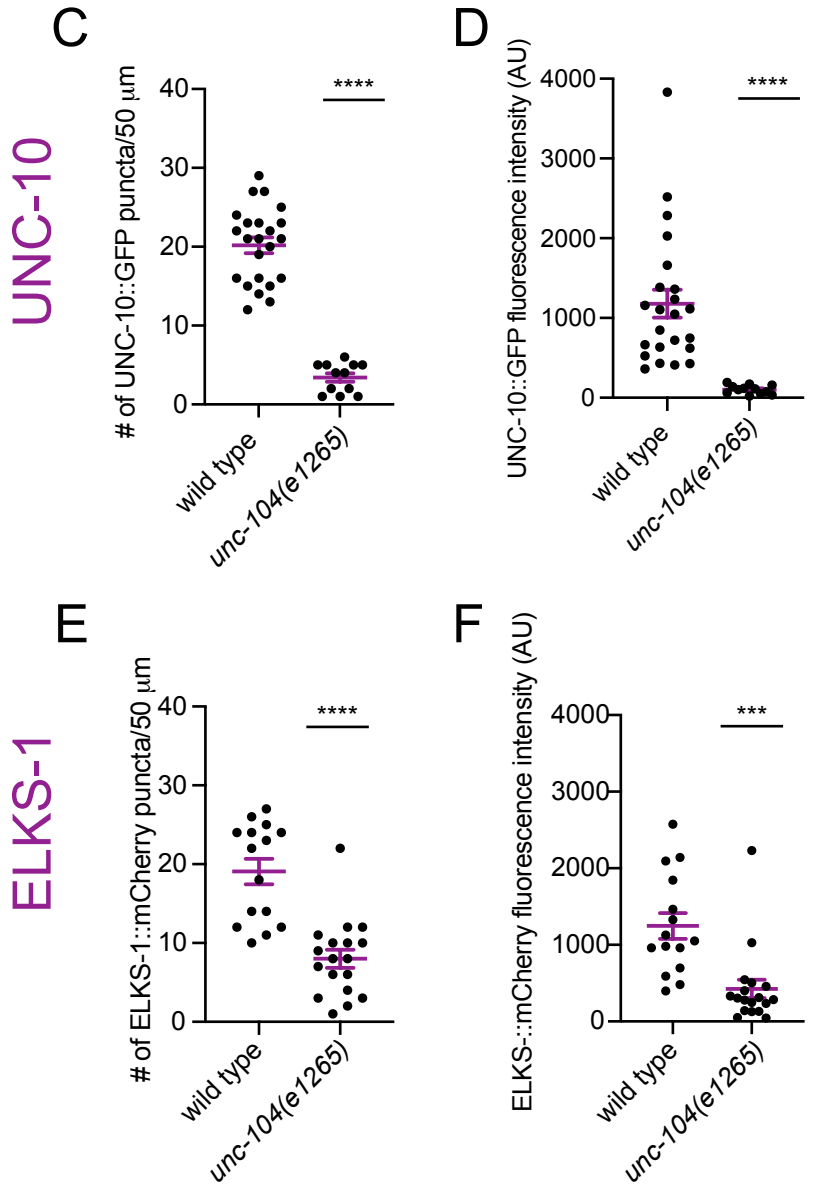
Figure S4.7. Active zone protein localization in unc-104(e1265) mutants.

(A) Scatterplot of CLA-1::GFP puncta number in a $50 \mu \mathrm{m}$ region of the dorsal nerve cord in wild type and unc-104(e1265) animals. Student's t-test, ${ }^{* * *} p<0.0001$. Bars, mean \pm SEM. These data correspond to quantification in Figure 4C.

(B) Scatterplot of CLA-1::GFP fluorescence intensity in a $50 \mu \mathrm{m}$ region of the dorsal nerve cord in wild type and unc-104(e1265) animals. Student's t-test, ${ }^{* * * *} p<0.0001$. Bars, mean \pm SEM.

(C) Scatterplot of UNC-10::GFP puncta number in a $50 \mu \mathrm{m}$ region of the dorsal nerve cord in wild type and unc-104(e1265) animals. Student's t-test, ${ }^{* * * *} p<0.0001$. Bars, mean \pm SEM. These data correspond to quantification in Figure 4C.

(D) Scatterplot of UNC-10::GFP fluorescence intensity in a $50 \mu \mathrm{m}$ region of the dorsal nerve cord in wild type and unc-104(e1265) mutants. Student's t-test, ${ }^{* * * *} p<0.0001$. Bars, mean \pm SEM.

(E) Scatterplot of ELKS-1::mCherry puncta number per $50 \mu \mathrm{m}$ of the dorsal nerve cord in wild type and unc-104(e1265) animals. Student's t-test, ${ }^{* * *} p<0.0001$. Bars, mean \pm SEM. These data correspond to quantification in Figure 4C.

(F) Scatterplot of ELKS-1::mCherry fluorescence intensity in a $50 \mu \mathrm{m}$ region of the dorsal nerve cords of wild type and unc-104(e1265) mutants. Student's t-test, ${ }^{* * *} p<0.001$. Bars, mean \pm SEM . 

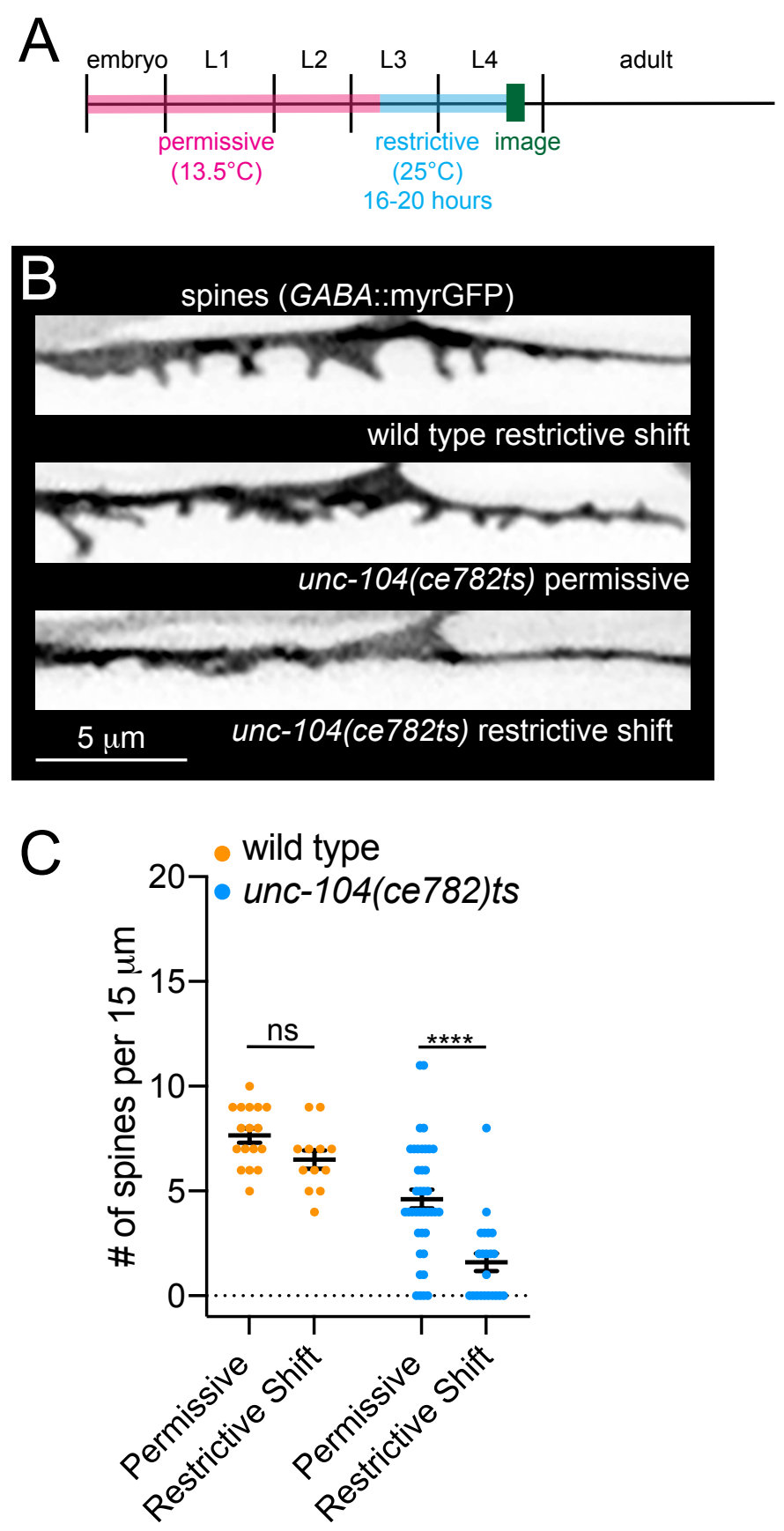
Figure S5.1 Shift to restrictive temperature during L3 in wild type and unc-104(ce782)ts animals.

(A) Cartoon depiction of experimental timeline. Animals were grown at $13.5^{\circ} \mathrm{C}$ until L3 stage ( approx. 120 hours in unc-104(ce782) mutants, 96 hours in wild type animals) before shifting animals to their restrictive temperature of $25^{\circ} \mathrm{C}$ for $16-20$ hours and imaging.

(B) Fluorescent images of DD spines (Pflp-13::myrGFP) from wild type (top) or unc104(ce782)ts animals grown continuously at the permissive temperature $\left(13.5^{\circ} \mathrm{C}\right)$ (middle panel) or shifted to the restrictive temperature $\left(25^{\circ} \mathrm{C}\right)$ for $16-20$ hours before imaging.

(C) Quantification of DD spines per $15 \mu \mathrm{m}$ from wild type and unc-104(ce782)ts mutants. Twoway ANOVA, Tukey's multiple comparisons test, ${ }^{* * *} p<0.0001, \mathrm{n} \geq 12$ animals. Data points indicate mean \pm SEM. 